Supporting Information

\title{
Trifluoromethylation of Benzoic Acids: an Access to Aryl Trifluoromethyl Ketones
}

Xue Liu, Long Liu, Tianzeng Huang, Jingjing Zhang, Zhi Tang, Chunya Li, Tieqiao Chen*

Key Laboratory of Ministry of Education for Advanced Materials in Tropical Island Resources, Hainan Provincial Key Lab of Fine Chem, Hainan Provincial Fine Chemical Engineering Research Center, Hainan University, Haikou, 570228, China.

Correspondence to:

E-mail: chentieqiao@hnu.edu.cn 


\section{Table of Contents}

1 General Information $\quad$ S3

2 Experimental Procedure $\quad$ S4

$\begin{array}{lll}\text { Characterization Data for the Products } & \text { S7 }\end{array}$

$\begin{array}{llr}4 & \text { References } & \text { S17 }\end{array}$

5 Copies of ${ }^{1} \mathrm{H},{ }^{13} \mathrm{C}$ and ${ }^{19} \mathrm{~F}$ NMR Spectra of the Products $\quad$ S18 


\section{General Information}

All experiments were carried out under a $\mathrm{N}_{2}$ atmosphere using standard Schlenk techniques or in a dry glovebox. All heating (heating module) and stirring were conducted on the IKA (Model: RCT B S025). Solvents were dried over $\mathrm{Na}$ metal or $\mathrm{CaH}_{2}$, and were distilled under nitrogen prior to use. Reagents were of analytical grade, obtained from commercial suppliers and used without further purification. Column chromatography was performed using Silica Gel 60 (200-300 mesh). The reactions were monitored by GC and GC-MS, GC-MS data were recorded on GC-MS QP 2010 plus, and GC analysis was performed on GC $2014 .{ }^{1} \mathrm{H}$ NMR $(400 \mathrm{MHz})$ spectra were recorded on Bruker ADVANCE III spectrometers in $\mathrm{CDCl}_{3}$ [using $\left(\mathrm{CH}_{3}\right)_{4} \mathrm{Si}$ (for ${ }^{1} \mathrm{H}, \delta=0.00$ ) as internal standard]. ${ }^{13} \mathrm{C}$ NMR $(100 \mathrm{MHz})$ spectra on Bruker ADVANCE III spectrometers in $\mathrm{CDCl}_{3}$ [using $\mathrm{CDCl}_{3}$ (for ${ }^{13} \mathrm{C}, \delta=$ 77.00) as an internal standard]. The following abbreviations were used to explain the multiplicities: $\mathrm{s}$ (singlet), d (doublet), dd (doublet of doublets), t (triplet), q (quartet), m (multiple), td (triplet of doublets). Chemical shifts $(\delta)$ are in parts per million relatives to $\mathrm{CDCl}_{3}$ at $7.26 \mathrm{ppm}$ for ${ }^{1} \mathrm{H}$ and at 77.16 ppm for ${ }^{13} \mathrm{C}\{1 \mathrm{H}\},{ }^{19} \mathrm{~F}$ NMR spectra were recorded using the F-H decoupled pulse sequence from the Varian program library. Melting points were measured using a melting point instrument and are uncorrected. High-resolution mass spectra (HRMS) were recorded on a TOF instrument by the ESI technique. All solvents, reagents and acid were purchased from Energy Chemical, Alfa Aesar and Aladdin. 


\section{Experimental Procedure}

\subsection{Optimization of reaction conditions.}

Table S1. Optimization of Reaction Conditions on the Trifluoromethylation of 4-Phenyl Benzoic Acid with $\mathrm{TMSCF}_{3 .}{ }^{[\mathrm{a}]}$

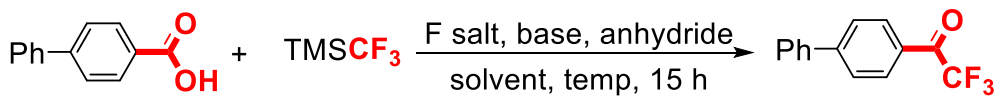

1a 2

\begin{tabular}{|c|c|c|c|c|c|c|}
\hline entry & $\begin{array}{l}\text { anhydride } \\
\text { (X equiv) }\end{array}$ & $\begin{array}{c}\text { base } \\
\text { (2.5 equiv) }\end{array}$ & $\begin{array}{c}\text { F salt } \\
\text { (2.5 equiv) }\end{array}$ & $\begin{array}{l}\text { Solvent } \\
(2.0 \mathrm{ml})\end{array}$ & $\begin{array}{l}\text { temp } \\
\left({ }^{\circ} \mathrm{C}\right)\end{array}$ & yield $(\%)$ \\
\hline 1 & TFAA (2.0 equiv.) & DMAP & $\mathrm{CsF}$ & $\mathrm{PhOMe}$ & 120 & $90(86)^{b}$ \\
\hline 2 & TFAA (1.0 equiv.) & DMAP & $\mathrm{CsF}$ & PhOMe & 120 & 13 \\
\hline 3 & TFAA ( 1.5 equiv.) & DMAP & $\mathrm{CsF}$ & PhOMe & 120 & 72 \\
\hline 4 & TFAA ( 2.5 equiv.) & DMAP & $\mathrm{CsF}$ & PhOMe & 120 & 71 \\
\hline 5 & TFAA (3.0 equiv.) & DMAP & $\mathrm{CsF}$ & PhOMe & 120 & 75 \\
\hline 6 & -- & DMAP & CsF & PhOMe & 120 & N.D. \\
\hline 7 & $\mathrm{Piv}_{2} \mathrm{O}$ (2.0 equiv.) & DMAP & $\mathrm{CsF}$ & PhOMe & 120 & N.D. \\
\hline 8 & $\mathrm{Ac}_{2} \mathrm{O}$ (2.0 equiv.) & DMAP & $\mathrm{CsF}$ & PhOMe & 120 & N.D. \\
\hline 9 & $\mathrm{Boc}_{2} \mathrm{O}$ (2.0 equiv.) & DMAP & $\mathrm{CsF}$ & PhOMe & 120 & N.D. \\
\hline 10 & $\mathrm{Tf}_{2} \mathrm{O}$ (2.0 equiv.) & DMAP & $\mathrm{CsF}$ & PhOMe & 120 & 41 \\
\hline 11 & $\mathrm{Ms}_{2} \mathrm{O}$ (2.0 equiv.) & DMAP & $\mathrm{CsF}$ & PhOMe & 120 & 19 \\
\hline 12 & TFAA (2.0 equiv.) & -- & CsF & PhOMe & 120 & N.D. \\
\hline 13 & TFAA (2.0 equiv.) & $\mathrm{NaHCO}_{3}$ & $\mathrm{CsF}$ & PhOMe & 120 & N.D. \\
\hline 14 & TFAA (2.0 equiv.) & $\mathrm{CH}_{3} \mathrm{COONa} \cdot 3 \mathrm{H}_{2} \mathrm{O}$ & CsF & PhOMe & 120 & N.D. \\
\hline 15 & TFAA (2.0 equiv.) & $\mathrm{Na}_{2} \mathrm{CO}_{3}$ & CsF & PhOMe & 120 & trace \\
\hline 16 & TFAA (2.0 equiv.) & $\mathrm{Et}_{3} \mathrm{~N}$ & CsF & PhOMe & 120 & trace \\
\hline 17 & TFAA (2.0 equiv.) & DBU & $\mathrm{CsF}$ & PhOMe & 120 & trace \\
\hline 18 & TFAA (2.0 equiv.) & pyridine & $\mathrm{CsF}$ & PhOMe & 120 & trace \\
\hline 19 & TFAA (2.0 equiv.) & DMAP & -- & PhOMe & 120 & 80 \\
\hline 20 & TFAA (2.0 equiv.) & DMAP & $\mathrm{KF}$ & PhOMe & 120 & 80 \\
\hline 21 & TFAA (2.0 equiv.) & DMAP & $\mathrm{NaF}$ & $\mathrm{PhOMe}$ & 120 & 73 \\
\hline 22 & TFAA (2.0 equiv.) & DMAP & $\mathrm{LiF}$ & PhOMe & 120 & 63 \\
\hline 23 & TFAA (2.0 equiv.) & DMAP & $\left(\mathrm{C}_{4} \mathrm{H}_{9}\right)_{4} \mathrm{NF}$ & PhOMe & 120 & trace \\
\hline 24 & TFAA (2.0 equiv.) & DMAP & $\mathrm{CsF}$ & THF & 120 & 60 \\
\hline 25 & TFAA (2.0 equiv.) & DMAP & $\mathrm{CsF}$ & cyclohexane & 120 & 71 \\
\hline 26 & TFAA (2.0 equiv.) & DMAP & $\mathrm{CsF}$ & NMP & 120 & 28 \\
\hline 27 & TFAA (2.0 equiv.) & DMAP & $\mathrm{CsF}$ & $\mathrm{CH}_{3} \mathrm{CN}$ & 120 & 77 \\
\hline 28 & TFAA (2.0 equiv.) & DMAP & $\mathrm{CsF}$ & toluene & 120 & 64 \\
\hline 29 & TFAA (2.0 equiv.) & DMAP & $\mathrm{CsF}$ & 1,4-dioxane & 120 & 72 \\
\hline 30 & TFAA (2.0 equiv.) & DMAP & $\mathrm{CsF}$ & $\mathrm{PhOMe}$ & 100 & 80 \\
\hline 31 & TFAA (2.0 equiv.) & DMAP & $\mathrm{CsF}$ & PhOMe & 140 & 56 \\
\hline
\end{tabular}

[a]Acid 1a (0.2 mmol, 1.0 equiv.), 2 ( 0.6 mmol, 3.0 equiv.), TFAA (2.0 equiv.), CsF (2.5 equiv.), DMAP (2.5 equiv.), PhOMe (2.0 ml), in $\mathrm{N}_{2}, 120^{\circ} \mathrm{C}, 15 \mathrm{~h}$. GC yield using dodecane as an internal standard. TFAA: trifluoroacetic anhydride. DMAP: 4-dimethylaminopyridine. DBU: 1,8-Diazabicyclo[5.4.0]undec-7-ene. NMP: N-Methyl pyrrolidone. ${ }^{[b]}$ solated yield. 


\subsection{General Experimental Procedure for the Synthesis of Trifluoromethyl ketones.}

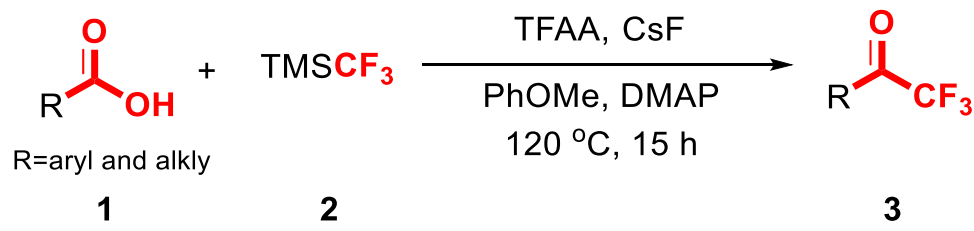

An oven dried $25 \mathrm{~mL}$ Schlenk tube was charged with carboxylic acid 1 (0.2 mmol), DMAP (0.5 mmol, 2.5 equiv.), $\mathrm{CsF}$ ( $0.5 \mathrm{mmol}, 2.5$ equiv.). Subsequently, $\mathrm{TMSCF}_{3} 2$ (0.6 mmol, 3.0 equiv.), TFAA (0.4 mmol, 2.0 equiv.) and $\mathrm{PhOMe}(2 \mathrm{~mL})$ were added under a $\mathrm{N}_{2}$ atmosphere. The reaction mixture was reacted at $120{ }^{\circ} \mathrm{C}$ for 15 hours. Then the mixture was cooled to room temperature, added water $(10 \mathrm{~mL})$, extracted with EtOAc $(5 \mathrm{~mL} \times 3)$. The combined organic phases were dried over anhydrous $\mathrm{Na}_{2} \mathrm{SO}_{4}$, filtered and concentrated under vacuum. Further purification by flash column chromatography on silica gel (eluting with petroleum ether/ethyl acetate) provided the product $\mathbf{3}$.

\subsection{Experimental Procedure for the trifluoromethylation of probenecid}

\subsubsection{Experimental Procedure for the trifluoromethylation of probenecid for $1 \mathrm{mmol}$ scale.}<smiles>CCCN(CCC)S(=O)(=O)c1ccc(C(=O)O)cc1</smiles>
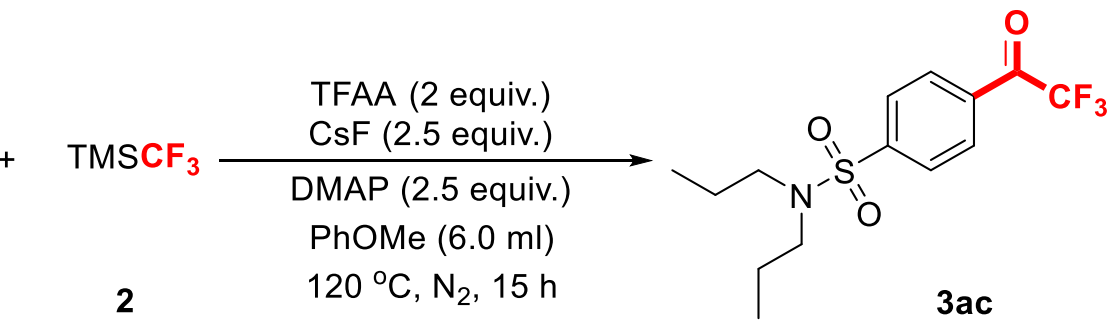

An oven dried $25 \mathrm{~mL}$ Schlenk tube was charged with carboxylic acid 1ac (1 mmol), DMAP (2.5 mmol, 2.5 equiv.), CsF ( $2.5 \mathrm{mmol}, 2.5$ equiv.). Subsequently, $\mathrm{TMSCF}_{3} 2$ (3 mmol, 3.0 equiv.), TFAA ( $2 \mathrm{mmol}$, 2.0 equiv.) and $\mathrm{PhOMe}(6 \mathrm{~mL})$ were added under a $\mathrm{N}_{2}$ atmosphere. The reaction mixture was reacted at $120{ }^{\circ} \mathrm{C}$ for 15 hours. Then the mixture was cooled to room temperature, added water $(40 \mathrm{~mL})$, extracted with EtOAc $(10 \mathrm{~mL} \times 3)$. The combined organic phases were dried over anhydrous $\mathrm{Na}_{2} \mathrm{SO}_{4}$, filtered and concentrated under vacuo. The desired product was isolated by column chromatography over silica gel (200-300 mesh) using petroleum ether/ethyl acetate $=3 / 1$ as eluent to afford a yellow solid 3ac in $87 \%$ yield $(293.2 \mathrm{mg})$.

\subsubsection{Experimental Procedure for the trifluoromethylation of probenecid for $2 \mathrm{mmol}$ scale.}

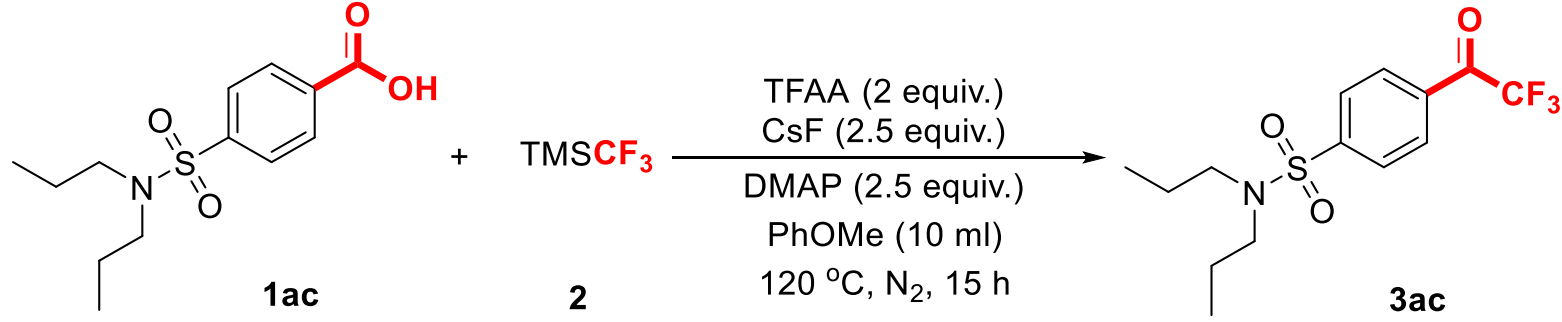

An oven dried $100 \mathrm{~mL}$ Schlenk tube was charged with carboxylic acid 1ac (2 mmol), DMAP (5 mmol, 2.5 equiv.), $\mathrm{CsF}$ (5 mmol, 2.5 equiv.). Subsequently, $\mathrm{TMSCF}_{3} 2$ (6 mmol, 3.0 equiv.), TFAA (4 mmol, 2.0 equiv.) and $\mathrm{PhOMe}(10 \mathrm{~mL})$ were added under a $\mathrm{N}_{2}$ atmosphere. The reaction mixture was reacted 
at $120{ }^{\circ} \mathrm{C}$ for 15 hours. Then the mixture was cooled to room temperature, added water $(60 \mathrm{~mL})$, extracted with EtOAc $(20 \mathrm{~mL} \times 3)$. The combined organic phases were dried over anhydrous $\mathrm{Na}_{2} \mathrm{SO}_{4}$, filtered and concentrated under vacuo. The desired product was isolated by column chromatography over silica gel (200-300 mesh) using petroleum ether/ethyl acetate $=3 / 1$ as eluent to afford a yellow solid 3ac in $86 \%$ yield $(579.6 \mathrm{mg})$.

\subsubsection{Experimental Procedure for the trifluoromethylation of probenecid for 5 mmol scale.}<smiles>CCCN(CCC)S(=O)(=O)c1ccc(C(=O)O)cc1</smiles><smiles>CCCN(CCCCCC(C)(C)C)S(=O)(=O)c1ccc(C(F)(F)F)cc1</smiles>

An oven dried $100 \mathrm{~mL}$ Schlenk tube was charged with carboxylic acid 1ac (5 mmol), DMAP (12.5 mmol, 2.5 equiv.), CsF (12.5 mmol, 2.5 equiv.). Subsequently, TMSCF 2 (15 mmol, 3.0 equiv.), TFAA (10 mmol, 2.0 equiv.) and PhOMe $(30 \mathrm{~mL})$ were added under a $\mathrm{N}_{2}$ atmosphere. The reaction mixture was reacted at $120{ }^{\circ} \mathrm{C}$ for 15 hours. Then the mixture was cooled to room temperature, added water $(120 \mathrm{~mL})$, extracted with EtOAc $(60 \mathrm{~mL} \times 3)$. The combined organic phases were dried over anhydrous $\mathrm{Na}_{2} \mathrm{SO}_{4}$, filtered and concentrated under vacuo. The desired product was isolated by column chromatography over silica gel (200-300 mesh) using petroleum ether/ethyl acetate $=3 / 1$ as eluent to afford a yellow solid 3ac in $84 \%$ yield (1.415 g). 


\section{Characterization Data for the Products}

\section{1-([1,1'-biphenyl]-4-yl)-2,2,2-trifluoroethan-1-one (3a)}<smiles>O=C(c1ccc(-c2ccccc2)cc1)C(F)(F)F</smiles>

The representative general procedure mentioned above was followed. Purification by preparative thin-layer chromatography (PTLC) on silica gel (petroleum ether/ethyl acetate $=10 / 1$ ) yielded the title compound 3a in $86 \%\left(86.0 \mathrm{mg}\right.$ ) as a white solid; $\mathrm{R}_{\mathrm{f}}=0.41$ (petroleum ether/ethyl acetate $\left.=10 / 1\right)$; $\mathrm{mp}$ : 50.2-51.9 ${ }^{\circ} \mathrm{C} ;{ }^{1} \mathrm{H}$ NMR $\left(400 \mathrm{MHz}, \mathrm{CDCl}_{3}\right) \delta 8.16(\mathrm{db}, J=8.2 \mathrm{~Hz}, 2 \mathrm{H}), 7.79-7.76(\mathrm{~m}, 2 \mathrm{H}), 7.67-7.64$ $(\mathrm{m}, 2 \mathrm{H}), 7.53-7.48(\mathrm{~m}, 2 \mathrm{H}), 7.47-7.43(\mathrm{~m}, 3 \mathrm{H}) .{ }^{13} \mathrm{C} \mathrm{NMR}\left(100 \mathrm{MHz}, \mathrm{CDCl}_{3}\right) \delta 180.1(\mathrm{q}, J=34.8 \mathrm{~Hz})$, $148.3,139.1,130.8(\mathrm{q}, J=2.0 \mathrm{~Hz}), 129.2,128.9,128.6,127.7,127.4,116.8(\mathrm{q}, J=289.6 \mathrm{~Hz}) .{ }^{19} \mathrm{~F} \mathrm{NMR}$ $\left(376 \mathrm{MHz}, \mathrm{CDCl}_{3}\right) \delta-71.30$. This compound is known. ${ }^{1}$

\section{1-([1,1'-biphenyl]-3-yl)-2,2,2-trifluoroethan-1-one (3b)}<smiles>O=C(c1cccc(-c2ccccc2)c1)C(F)(F)F</smiles>

The representative general procedure mentioned above was followed. Purification by preparative thin-layer chromatography (PTLC) on silica gel (petroleum ether/ethyl acetate $=10 / 1$ ) yielded the title compound $\mathbf{3 b}$ in $87 \%\left(87.0 \mathrm{mg}\right.$ ) as a white liquid; $\mathrm{R}_{\mathrm{f}}=0.45$ (petroleum ether/ethyl acetate $\left.=10 / 1\right) ;{ }^{1} \mathrm{H}$ NMR (400 MHz, $\left.\mathrm{CDCl}_{3}\right) \delta 8.30(\mathrm{~s}, 1 \mathrm{H}), 8.07-8.05(\mathrm{~m}, 1 \mathrm{H}), 7.95-7.93(\mathrm{~m}, 1 \mathrm{H}), 7.66-7.61(\mathrm{~m}, 3 \mathrm{H})$, 7.53-7.49 (m, 2H), 7.46-7.41 (m, 1H). $\left.{ }^{13} \mathrm{C} \mathrm{NMR} \mathrm{(100} \mathrm{MHz,} \mathrm{CDCl}_{3}\right) \delta 180.6(\mathrm{q}, J=34.8 \mathrm{~Hz}), 142.4$, 139.4, 134.2, 130.5, 129.6, 129.1, 128.8 (q, $J=2.1 \mathrm{~Hz}), 128.7(\mathrm{q}, J=1.8 \mathrm{~Hz}), 128.3,127.2,116.7(\mathrm{q}, J$ $=289.6 \mathrm{~Hz}) .{ }^{19} \mathrm{~F}$ NMR $\left(376 \mathrm{MHz}, \mathrm{CDCl}_{3}\right) \delta-71.24$. HRMS (ESI-TOF) m/z: [M] Calcd for $\mathrm{C}_{14} \mathrm{H}_{9} \mathrm{~F}_{3} \mathrm{O}$ 250.0605; Found 250.0609 for the compounds: $3 \mathbf{b}$.

\section{1-([1,1'-biphenyl]-2-yl)-2,2,2-trifluoroethan-1-one (3c)}<smiles>O=C(c1ccccc1-c1ccccc1)C(F)(F)F</smiles>

The representative general procedure mentioned above was followed. Purification by preparative thin-layer chromatography (PTLC) on silica gel (petroleum ether) yielded the title compound 3c in $68 \%$ $(68.0 \mathrm{mg})$ as a white liquid; $\mathrm{R}_{\mathrm{f}}=0.49$ (petroleum ether); ${ }^{1} \mathrm{H} \mathrm{NMR}\left(400 \mathrm{MHz}, \mathrm{CDCl}_{3}\right) \delta 7.73(\mathrm{~d}, J=8.1$ $\mathrm{Hz}, 1 \mathrm{H}), 7.66-7.62(\mathrm{~m}, 1 \mathrm{H}), 7.51-7.46(\mathrm{~m}, 2 \mathrm{H}), 7.43-7.35(\mathrm{~m}, 3 \mathrm{H}), 7.27-7.24(\mathrm{~m}, 2 \mathrm{H}) .{ }^{13} \mathrm{C} \mathrm{NMR}(100$ $\left.\mathrm{MHz}, \mathrm{CDCl}_{3}\right) \delta 185.7(\mathrm{q}, J=35.4 \mathrm{~Hz}), 143.4,139.7,132.9,131.9,131.4,128.9(\mathrm{q}, J=2.2 \mathrm{~Hz}), 128.7$, 128.6, 128.0, 127.4, 115.9 (q, $J=290.6 \mathrm{~Hz}) .{ }^{19} \mathrm{~F} \mathrm{NMR}\left(376 \mathrm{MHz}, \mathrm{CDCl}_{3}\right) \delta-73.01$. This compound is known. $^{2}$ 
<smiles>O=C(c1ccc(C2CCCCC2)cc1)C(F)(F)F</smiles>

The representative general procedure mentioned above was followed. Purification by preparative thin-layer chromatography (PTLC) on silica gel (petroleum ether) yielded the title compound 3d in 79\% $\left(80.9 \mathrm{mg}\right.$ ) as a yellow liquid; $\mathrm{R}_{\mathrm{f}}=0.32$ (petroleum ether); ${ }^{1} \mathrm{H} \mathrm{NMR}\left(400 \mathrm{MHz}, \mathrm{CDCl}_{3}\right) \delta 8.02-8.00(\mathrm{~m}$, 2H), 7.39-7.37 (m, 2H), 2.64-2.57 (m, 1H), 1.90-1.86 (m, 4H), 1.80-1.76 (m, 1H), 1.47-1.25 (m, 5H). ${ }^{13} \mathrm{C}$ NMR $\left(100 \mathrm{MHz}, \mathrm{CDCl}_{3}\right) \delta 180.1(\mathrm{q}, J=34.6 \mathrm{~Hz}), 156.7,130.4(\mathrm{q}, J=2.0 \mathrm{~Hz}), 127.7,127.7,116.8$ $(\mathrm{q}, J=289.7 \mathrm{~Hz}), 44.9,33.9,26.6,25.9 .{ }^{19} \mathrm{~F} \mathrm{NMR}\left(376 \mathrm{MHz}, \mathrm{CDCl}_{3}\right) \delta-71.28$. This compound is known. ${ }^{10}$

\section{1-(4-(tert-butyl)phenyl)-2,2,2-trifluoroethan-1-one (3e)}<smiles>CC(C)(C)c1ccc(C(=O)C(F)(F)F)cc1</smiles>

The representative general procedure mentioned above was followed. Purification by preparative thin-layer chromatography (PTLC) on silica gel (petroleum ether) yielded the title compound $\mathbf{3 e}$ in $80 \%$ (73.6 mg) as a white solid; $\mathrm{R}_{\mathrm{f}}=0.26$ (petroleum ether); mp: $38.0-39.8{ }^{\circ} \mathrm{C} ;{ }^{1} \mathrm{H} \mathrm{NMR}\left(400 \mathrm{MHz}, \mathrm{CDCl}_{3}\right)$ $\delta$ 8.04-8.01 (m, 2H), 7.60-7.55 (m, 2H), $1.36(\mathrm{~s}, 9 \mathrm{H}) .{ }^{13} \mathrm{C} \mathrm{NMR}\left(100 \mathrm{MHz}, \mathrm{CDCl}_{3}\right) \delta 180.1$ (q, $J=34.5$ $\mathrm{Hz}), 159.9,130.2(\mathrm{q}, J=2.0 \mathrm{~Hz}), 127.4,126.2,116.8(\mathrm{q}, J=289.7 \mathrm{~Hz}), 35.5,30.9 .{ }^{19} \mathrm{~F}$ NMR $(376 \mathrm{MHz}$, $\left.\mathrm{CDCl}_{3}\right) \delta-71.35$. This compound is known. ${ }^{4}$

\section{2,2,2-trifluoro-1-(4-methoxyphenyl)ethan-1-one (3f)}<smiles>COc1ccc(C(=O)C(F)(F)F)cc1</smiles>

The representative general procedure mentioned above was followed. Purification by preparative thin-layer chromatography (PTLC) on silica gel (petroleum ether/ethyl acetate $=100 / 1$ ) yielded the title compound $3 \mathbf{f}$ in $76 \%(62.0 \mathrm{mg})$ as a yellow liquid; $\mathrm{R}_{\mathrm{f}}=0.28$ (petroleum ether/ethyl acetate $\left.=100 / 1\right)$; ${ }^{1} \mathrm{H}$ NMR (400 MHz, $\left.\mathrm{CDCl}_{3}\right) \delta$ 8.06-8.04 (m, 2H), 7.02-6.98 (m, 2H), $3.91(\mathrm{~s}, 3 \mathrm{H}) .{ }^{13} \mathrm{C} \mathrm{NMR}(100 \mathrm{MHz}$, $\left.\mathrm{CDCl}_{3}\right) \delta 179.0(\mathrm{q}, J=34.2 \mathrm{~Hz}), 165.4,132.8(\mathrm{q}, J=2.0 \mathrm{~Hz}), 122.8,116.9(\mathrm{q}, J=289.7 \mathrm{~Hz}), 114.5$, 55.7. ${ }^{19} \mathrm{~F} \mathrm{NMR}\left(376 \mathrm{MHz}, \mathrm{CDCl}_{3}\right) \delta-71.02$. This compound is known. ${ }^{1}$

\section{2,2,2-trifluoro-1-(4-phenoxyphenyl)ethan-1-one (3g)}<smiles>O=C(c1ccc(Oc2ccccc2)cc1)C(F)(F)F</smiles>

The representative general procedure mentioned above was followed. Purification by preparative thin-layer chromatography (PTLC) on silica gel (petroleum ether/ethyl acetate $=5 / 1$ ) yielded the title 
compound $3 \mathrm{~g}$ in $74 \%(78.7 \mathrm{mg})$ as a yellow liquid; $\mathrm{R}_{\mathrm{f}}=0.68$ (petroleum ether/ethyl acetate $\left.=5 / 1\right) .{ }^{1} \mathrm{H}$ NMR (400 MHz, $\left.\mathrm{CDCl}_{3}\right) \delta$ 8.00-7.98 (m, 2H), 7.37-7.29 (m, 2H), 7.19-7.14 (m, 1H), 7.03-6.94 (m, $4 \mathrm{H}) .{ }^{13} \mathrm{C}$ NMR $\left(100 \mathrm{MHz}, \mathrm{CDCl}_{3}\right) \delta 179.0(\mathrm{q}, J=34.6 \mathrm{~Hz}), 164.3,154.5,132.8(\mathrm{q}, J=2.0 \mathrm{~Hz}), 130.3$, 125.4, 124.1, 120.7, 117.3, $116.8(\mathrm{q}, J=289.5 \mathrm{~Hz}) .{ }^{19} \mathrm{~F}$ NMR $\left(376 \mathrm{MHz}, \mathrm{CDCl}_{3}\right) \delta-71.13$. This compound is known. ${ }^{3}$

1-(4-(dimethylamino)phenyl)-2,2,2-trifluoroethan-1-one (3h)<smiles>CN(C)c1ccc(C(=O)C(F)(F)F)cc1</smiles>

The representative general procedure mentioned above was followed. Purification by preparative thin-layer chromatography (PTLC) on silica gel (petroleum ether/ethyl acetate $=40 / 1$ ) yielded the title compound $3 \mathbf{h}$ in $68 \%\left(59.0 \mathrm{mg}\right.$ ) as a yellow solid; $\mathrm{R}_{\mathrm{f}}=0.23$ (petroleum ether/ethyl acetate $\left.=40 / 1\right)$; $\mathrm{mp}$ : 75.3-76.6 ${ }^{\circ} \mathrm{C} ;{ }^{1} \mathrm{H}$ NMR $\left(400 \mathrm{MHz}, \mathrm{CDCl}_{3}\right) \delta$ 7.96-7.94 (m, 2H), 6.68-6.66 (m, 2H), $3.11(\mathrm{~s}, 6 \mathrm{H}) .{ }^{13} \mathrm{C}$ NMR $\left(100 \mathrm{MHz}, \mathrm{CDCl}_{3}\right) \delta 177.9(\mathrm{q}, J=33.4 \mathrm{~Hz}), 154.7,132.6(\mathrm{q}, J=2.1 \mathrm{~Hz}), 117.5(\mathrm{q}, J=289.8 \mathrm{~Hz})$, 117.4, 110.9, 40.0. ${ }^{19} \mathrm{~F}$ NMR $\left(376 \mathrm{MHz}, \mathrm{CDCl}_{3}\right) \delta-70.30$. This compound is known. ${ }^{9}$

\section{2,2,2-trifluoro-1-(4-iodophenyl)ethan-1-one (3i)}<smiles>O=C(c1ccc(I)cc1)C(F)(F)F</smiles>

The representative general procedure mentioned above was followed. Purification by preparative thin-layer chromatography (PTLC) on silica gel (petroleum ether/ethyl acetate $=5 / 1$ ) yielded the title compound 3i in 89\% (106.8 mg) as a yellow liquid; $\mathrm{R}_{\mathrm{f}}=0.39$ (petroleum ether/ethyl acetate $\left.=5 / 1\right) ;{ }^{1} \mathrm{H}$ $\operatorname{NMR}\left(400 \mathrm{MHz}, \mathrm{CDCl}_{3}\right) \delta$ 7.93-7.90 (m, 2H), 7.76-7.73 (m, 2H). ${ }^{13} \mathrm{C} \mathrm{NMR}\left(100 \mathrm{MHz}, \mathrm{CDCl}_{3}\right) \delta 180.0$ $(\mathrm{q}, J=35.3 \mathrm{~Hz}), 138.6,131.1(\mathrm{q}, J=2.1 \mathrm{~Hz}), 129.1,116.5(\mathrm{q}, J=289.4 \mathrm{~Hz}), 104.7 .{ }^{19} \mathrm{~F}$ NMR $(376$ $\left.\mathrm{MHz}, \mathrm{CDCl}_{3}\right) \delta-71.57$. This compound is known. ${ }^{1}$

\section{2,2,2-trifluoro-1-(2-iodophenyl)ethan-1-one (3j)}<smiles>O=C(c1ccccc1I)C(F)(F)F</smiles>

The representative general procedure mentioned above was followed. Purification by preparative thin-layer chromatography (PTLC) on silica gel (petroleum ether) yielded the title compound $\mathbf{3 j}$ in $72 \%$ $(86.4 \mathrm{mg})$ as a yellow liquid; $\mathrm{R}_{\mathrm{f}}=0.41$ (petroleum ether); ${ }^{1} \mathrm{H} \mathrm{NMR}\left(400 \mathrm{MHz}, \mathrm{CDCl}_{3}\right) \delta 8.10(\mathrm{dd}, J=$ 8.0, $1.0 \mathrm{~Hz}, 1 \mathrm{H}), 7.75-7.71(\mathrm{~m}, 1 \mathrm{H}), 7.53-7.49(\mathrm{~m}, 1 \mathrm{H}), 7.30-7.27(\mathrm{~m}, 1 \mathrm{H}) .{ }^{13} \mathrm{C} \mathrm{NMR}(100 \mathrm{MHz}$, $\left.\mathrm{CDCl}_{3}\right) \delta 182.5(\mathrm{q}, J=36.0 \mathrm{~Hz}), 142.4,134.5,134.2,130.1(\mathrm{q}, J=3.2 \mathrm{~Hz}), 128.1,115.5(\mathrm{q}, J=290.3$ $\mathrm{Hz}$ ), 94.0. ${ }^{19} \mathrm{~F}$ NMR $\left(376 \mathrm{MHz}, \mathrm{CDCl}_{3}\right) \delta$-72.31. HRMS (ESI-TOF) $\mathrm{m} / \mathrm{z}:[\mathrm{M}+\mathrm{H}]^{+} \mathrm{Calcd}$ for $\mathrm{C}_{8} \mathrm{H}_{4} \mathrm{~F}_{3} \mathrm{IO}+\mathrm{H} 300.9332$; Found 300.9343 for the compounds: $\mathbf{3 j}$. 
<smiles>O=C(c1ccc([N+](=O)[O-])cc1)C(F)(F)F</smiles>

The representative general procedure mentioned above was followed. Purification by preparative thin-layer chromatography (PTLC) on silica gel (petroleum ether/ethyl acetate $=3 / 1$ ) yielded the title compound 3k in $75 \%\left(65.7 \mathrm{mg}\right.$ ) as a light yellow solid; $\mathrm{R}_{\mathrm{f}}=0.37$ (petroleum ether/ethyl acetate $\left.=3 / 1\right)$; mp: 94.6-95.3 ${ }^{\circ} \mathrm{C} ;{ }^{1} \mathrm{H}$ NMR $\left(400 \mathrm{MHz}, \mathrm{CDCl}_{3}\right) \delta 8.42-8.39(\mathrm{~m}, 2 \mathrm{H}), 8.28-8.25(\mathrm{~m}, 2 \mathrm{H}) .{ }^{13} \mathrm{C} \mathrm{NMR}(100$ $\left.\mathrm{MHz}, \mathrm{CDCl}_{3}\right) \delta 179.3(\mathrm{q}, J=36.5 \mathrm{~Hz}), 151.5,134.2,131.3(\mathrm{q}, J=2.0 \mathrm{~Hz}), 124.2,116.2(\mathrm{q}, J=289.3$ $\mathrm{Hz}) .{ }^{19} \mathrm{~F}$ NMR $\left(376 \mathrm{MHz}, \mathrm{CDCl}_{3}\right) \delta-71.87$. This compound is known. ${ }^{7}$

\section{2,2,2-trifluoro-1-(4-isocyanophenyl)ethan-1-one (3I)}<smiles>N#Cc1ccc(C(=O)C(F)(F)F)cc1</smiles>

The representative general procedure mentioned above was followed. Purification by preparative thin-layer chromatography (PTLC) on silica gel (petroleum ether/ethyl acetate $=3 / 1$ ) yielded the title compound 31 in $81 \%\left(64.5 \mathrm{mg}\right.$ ) as a yellow solid; $R_{\mathrm{f}}=0.35$ (petroleum ether/ethyl acetate $\left.=3 / 1\right)$; $\mathrm{mp}$ : 76.6-77.8 ${ }^{\circ} \mathrm{C} ;{ }^{1} \mathrm{H}$ NMR $\left(400 \mathrm{MHz}, \mathrm{CDCl}_{3}\right) \delta 8.19-8.16(\mathrm{~m}, 2 \mathrm{H}), 7.88-7.85(\mathrm{~m}, 2 \mathrm{H}) .{ }^{13} \mathrm{C} \mathrm{NMR}(100$ $\left.\mathrm{MHz}, \mathrm{CDCl}_{3}\right) \delta 179.5(\mathrm{q}, J=36.2 \mathrm{~Hz}), 132.8,132.1,130.4(\mathrm{q}, J=2.1 \mathrm{~Hz}), 118.7,117.2,116.2(\mathrm{q}, J=$ $289.0 \mathrm{~Hz}) .{ }^{19} \mathrm{~F} \mathrm{NMR}\left(376 \mathrm{MHz}, \mathrm{CDCl}_{3}\right) \delta-71.79$. This compound is known. ${ }^{1}$

\section{1-(4-benzoylphenyl)-2,2,2-trifluoroethan-1-one (3m)}<smiles>O=C(c1ccccc1)c1ccc(C(=O)C(F)(F)F)cc1</smiles>

The representative general procedure mentioned above was followed. Purification by preparative thin-layer chromatography (PTLC) on silica gel (petroleum ether/ethyl acetate $=3 / 1$ ) yielded the title compound $3 \mathrm{~m}$ in $72 \%\left(80.1 \mathrm{mg}\right.$ ) as a white solid; $\mathrm{R}_{\mathrm{f}}=0.34$ (petroleum ether/ethyl acetate $\left.=3 / 1\right)$; $\mathrm{mp}$ : 81.6-81.9 ${ }^{\circ} \mathrm{C} ;{ }^{1} \mathrm{H}$ NMR $\left(400 \mathrm{MHz}, \mathrm{CDCl}_{3}\right) \delta 8.19(\mathrm{~d}, J=7.9 \mathrm{~Hz}, 2 \mathrm{H}), 7.93-7.91(\mathrm{~m}, 2 \mathrm{H}), 7.82-7.80(\mathrm{~m}$, 2H), 7.67-.65 (m, 1H), $7.51(\mathrm{dd}, J=10.6,4.7 \mathrm{~Hz}, 2 \mathrm{H}) .{ }^{13} \mathrm{C} \mathrm{NMR}\left(100 \mathrm{MHz}, \mathrm{CDCl}_{3}\right) \delta 195.4,180.1$ (q, $J=35.5 \mathrm{~Hz}), 143.4,136.4,133.4,132.2,130.2,130.0(\mathrm{q}, J=1.9 \mathrm{~Hz}), 128.7,116.5(\mathrm{q}, J=289.5 \mathrm{~Hz}) .{ }^{19} \mathrm{~F}$ NMR (376 MHz, $\left.\mathrm{CDCl}_{3}\right) \delta$-71.63. HRMS (ESI-TOF) m/z: [M] Calcd for $\mathrm{C}_{15} \mathrm{H}_{9} \mathrm{~F}_{3} \mathrm{O}_{2}$ 278.0555; Found 278.0555 for the compounds: $\mathbf{3 m}$.

\section{2,2,2-trifluoro-1-(4-methoxy-2-methylphenyl)ethan-1-one (3n)}<smiles>COc1ccc(C(=O)C(F)(F)F)c(C)c1</smiles>

The representative general procedure mentioned above was followed. Purification by preparative thin-layer chromatography (PTLC) on silica gel (petroleum ether) yielded the title compound 3n in 71\% 
$(61.9 \mathrm{mg})$ as a yellow liquid; $\mathrm{R}_{\mathrm{f}}=0.61$ (petroleum ether); ${ }^{1} \mathrm{H} \mathrm{NMR}\left(400 \mathrm{MHz}, \mathrm{CDCl}_{3}\right) \delta 7.92-7.89(\mathrm{~m}$, $1 \mathrm{H}), 6.84-6.81(\mathrm{~m}, 2 \mathrm{H}), 3.89(\mathrm{~s}, 3 \mathrm{H}), 2.61(\mathrm{~s}, 3 \mathrm{H}) .{ }^{13} \mathrm{C} \mathrm{NMR}\left(100 \mathrm{MHz}, \mathrm{CDCl}_{3}\right) \delta 179.1(\mathrm{q}, J=33.0$ $\mathrm{Hz}), 162.9,145.5,132.8$ (q, $J=4.0 \mathrm{~Hz}), 120.6,117.3,115.8(\mathrm{q}, J=291.4 \mathrm{~Hz}), 110.1,54.5,21.9 .{ }^{19} \mathrm{~F}$ NMR (376 MHz, $\left.\mathrm{CDCl}_{3}\right) \delta-70.31$. HRMS (ESI-TOF) m/z: $[\mathrm{M}+\mathrm{H}]^{+}$Calcd for $\mathrm{C}_{10} \mathrm{H}_{9} \mathrm{~F}_{3} \mathrm{O}_{2}+\mathrm{H} 219.0627$; Found 219.0642 for the compounds: $3 \mathbf{n}$.

\section{1-(3-chloro-5-methoxyphenyl)-2,2,2-trifluoroethan-1-one (3o)}<smiles>COc1cc(Cl)cc(C(=O)C(F)(F)F)c1</smiles>

The representative general procedure mentioned above was followed. Purification by preparative thin-layer chromatography (PTLC) on silica gel (petroleum ether/ethyl acetate $=5 / 1$ ) yielded the title compound 3o in 99\% (94.2 mg) as a yellow liquid; $\mathrm{R}_{\mathrm{f}}=0.43$ (petroleum ether/ethyl acetate $\left.=5 / 1\right) ;{ }^{1} \mathrm{H}$ $\operatorname{NMR}\left(400 \mathrm{MHz}, \mathrm{CDCl}_{3}\right) \delta 7.61(\mathrm{q}, J=1.1 \mathrm{~Hz}, 1 \mathrm{H}), 7.45(\mathrm{~s}, 1 \mathrm{H}), 7.23-7.22(\mathrm{~m}, 1 \mathrm{H}), 3.87(\mathrm{~s}, 3 \mathrm{H}) .{ }^{13} \mathrm{C}$ $\operatorname{NMR}\left(100 \mathrm{MHz}, \mathrm{CDCl}_{3}\right) \delta 178.4(\mathrm{q}, J=35.7 \mathrm{~Hz}), 159.6,135.0,130.8,121.4(\mathrm{q}, J=2.7 \mathrm{~Hz}), 120.6$, 115.4 (q, $J=289.2 \mathrm{~Hz}$ ), 112.4 (q, $J=1.8 \mathrm{~Hz}$ ), 54.9. HRMS (ESI-TOF) m/z: [M] Calcd for $\mathrm{C}_{9} \mathrm{H}_{6} \mathrm{ClF}_{3} \mathrm{O}_{2}$ 238.0008; Found 238.0007 for the compounds: 30.

\section{1-(benzo[d][1,3]dioxol-5-yl)-2,2,2-trifluoroethan-1-one (3p)}<smiles>O=C(c1ccc2c(c1)OCO2)C(F)(F)F</smiles>

The representative general procedure mentioned above was followed. Purification by preparative thin-layer chromatography (PTLC) on silica gel (petroleum ether/ethyl acetate $=20 / 1$ ) yielded the title compound $3 p$ in $63 \%(55.0 \mathrm{mg})$ as a yellow liquid; $\mathrm{R}_{\mathrm{f}}=0.46$ (petroleum ether/ethyl acetate $\left.=20 / 1\right) ;{ }^{1} \mathrm{H}$ NMR (400 MHz, $\left.\mathrm{CDCl}_{3}\right) \delta 7.72-7.70(\mathrm{~m}, 1 \mathrm{H}), 7.50(\mathrm{~b}, 1 \mathrm{H}), 6.93(\mathrm{~d}, J=8.3 \mathrm{~Hz}, 1 \mathrm{H}), 6.11(\mathrm{~s}, 2 \mathrm{H}) .{ }^{13} \mathrm{C}$ $\operatorname{NMR}\left(100 \mathrm{MHz}, \mathrm{CDCl}_{3}\right) \delta 178.6(\mathrm{q}, J=34.6 \mathrm{~Hz}), 154.0,148.6,127.7(\mathrm{q}, J=2.8 \mathrm{~Hz}), 124.4,116.8(\mathrm{q}$, $J=288.5 \mathrm{~Hz}), 109.3(\mathrm{q}, J=1.8 \mathrm{~Hz}), 108.5,102.5 .{ }^{19} \mathrm{~F} \mathrm{NMR}\left(376 \mathrm{MHz}, \mathrm{CDCl}_{3}\right) \delta-70.69$. This compound is known. ${ }^{8}$

\section{2,2,2-trifluoro-1-(3,4,5-trimethoxyphenyl)ethan-1-one (3q)}<smiles>COc1cc(C(=O)C(F)(F)F)cc(OC)c1OC</smiles>

The representative general procedure mentioned above was followed. Purification by preparative thin-layer chromatography (PTLC) on silica gel (petroleum ether/ethyl acetate $=5 / 1$ ) yielded the title compound 3q in $70 \%\left(73.9 \mathrm{mg}\right.$ ) as a white solid; $\mathrm{R}_{\mathrm{f}}=0.50$ (petroleum ether/ethyl acetate $\left.=5 / 1\right)$; $\mathrm{mp}$ : 80.5-81.5 ${ }^{\circ} \mathrm{C} ;{ }^{1} \mathrm{H}$ NMR (400 MHz, $\left.\mathrm{CDCl}_{3}\right) \delta 7.31$ (b, 2H), $3.97(\mathrm{~s}, 3 \mathrm{H}), 3.92(\mathrm{~s}, 6 \mathrm{H}) .{ }^{13} \mathrm{C}$ NMR $(100$ $\left.\mathrm{MHz}, \mathrm{CDCl}_{3}\right) \delta 179.2(\mathrm{q}, J=34.5 \mathrm{~Hz}), 153.2,144.9,124.6,116.8(\mathrm{q}, J=289.5 \mathrm{~Hz}), 107.6(\mathrm{q}, J=2.2$ $\mathrm{Hz}), 61.2,56.4 .{ }^{19} \mathrm{~F} \mathrm{NMR}\left(376 \mathrm{MHz}, \mathrm{CDCl}_{3}\right) \delta-70.49$. This compound is known. ${ }^{1}$ 


\section{2,2,2-trifluoro-1-(naphthalen-1-yl)ethan-1-one (3r)}<smiles>O=C(c1cccc2ccccc12)C(F)(F)C(F)(F)F</smiles>

The representative general procedure mentioned above was followed. Purification by preparative thin-layer chromatography (PTLC) on silica gel (petroleum ether/ethyl acetate $=20 / 1$ ) yielded the title compound $3 \mathbf{r}$ in $64 \%\left(57.3 \mathrm{mg}\right.$ ) as a white oil; $\mathrm{R}_{\mathrm{f}}=0.72$ (petroleum ether/ethyl acetate $\left.=20 / 1\right) ;{ }^{1} \mathrm{H}$ NMR (400 MHz, $\left.\mathrm{CDCl}_{3}\right) \delta 8.85(\mathrm{db}, J=8.8 \mathrm{~Hz}, 1 \mathrm{H}), 8.22-8.19(\mathrm{~m}, 1 \mathrm{H}), 8.16(\mathrm{~d}, J=8.2 \mathrm{~Hz}, 1 \mathrm{H}), 7.94$ $(\mathrm{d}, J=8.1 \mathrm{~Hz}, 1 \mathrm{H}), 7.73-7.69(\mathrm{~m}, 1 \mathrm{H}), 7.64-7.56(\mathrm{~m}, 2 \mathrm{H}) .{ }^{13} \mathrm{C} \mathrm{NMR}\left(100 \mathrm{MHz}, \mathrm{CDCl}_{3}\right) \delta 182.4(\mathrm{q}, J=$ $33.5 \mathrm{~Hz}), 136.3,134.0,131.7$ (q, $J=3.8 \mathrm{~Hz}), 131.2,129.6,129.0,127.2,126.4,125.2,124.2,116.7$ (q, $J=291.3 \mathrm{~Hz}) .{ }^{19} \mathrm{~F}$ NMR $\left(376 \mathrm{MHz}, \mathrm{CDCl}_{3}\right) \delta-70.14$. This compound is known. ${ }^{4}$

\section{2,2,2-trifluoro-1-(naphthalen-2-yl)ethan-1-one（3s）}<smiles>O=C(c1ccc2ccccc2c1)C(F)(F)F</smiles>

The representative general procedure mentioned above was followed. Purification by preparative thin-layer chromatography (PTLC) on silica gel (petroleum ether/dichloromethane $=20 / 1$ ) yielded the title compound $3 \mathrm{~s}$ in $73 \%(65.4 \mathrm{mg})$ as a light yellow solid; $\mathrm{R}_{\mathrm{f}}=0.44$ (petroleum ether/dichloromethane = 20/1); mp: 36.8-38.2 ${ }^{\circ} \mathrm{C} ;{ }^{1} \mathrm{H}$ NMR (400 MHz, $\left.\mathrm{CDCl}_{3}\right) \delta 8.63(\mathrm{~s}, 1 \mathrm{H}), 8.07(\mathrm{db}, J=8.7 \mathrm{~Hz}, 1 \mathrm{H}), 8.02$ $(\mathrm{d}, J=8.2 \mathrm{~Hz}, 1 \mathrm{H}), 7.96(\mathrm{~d}, J=8.7 \mathrm{~Hz}, 1 \mathrm{H}), 7.91(\mathrm{~d}, J=8.2 \mathrm{~Hz}, 1 \mathrm{H}), 7.72-7.68(\mathrm{~m}, 1 \mathrm{H}), 7.64-7.60(\mathrm{~m}$, $1 \mathrm{H}) .{ }^{13} \mathrm{C}$ NMR $\left(100 \mathrm{MHz}, \mathrm{CDCl}_{3}\right) \delta 180.5$ (q, $\left.J=35.0 \mathrm{~Hz}\right), 136.5,133.2(\mathrm{q}, J=3.0 \mathrm{~Hz}), 132.2,130.2$, 130.1, 129.1, 127.9, 127.5, 127.2, 124.2, 116.9 (q, $J=289.6 \mathrm{~Hz}) .{ }^{19} \mathrm{~F} \mathrm{NMR}\left(376 \mathrm{MHz}, \mathrm{CDCl}_{3}\right) \delta-70.72$. This compound is known. ${ }^{1}$

\section{2,2,2-trifluoro-1-(6-methoxynaphthalen-2-yl)ethan-1-one (3t)}<smiles>COc1ccc2cc(C(=O)C(F)(F)F)ccc2c1</smiles>

The representative general procedure mentioned above was followed. Purification by preparative thin-layer chromatography (PTLC) on silica gel (petroleum ether/ethyl acetate $=20 / 1$ ) yielded the title compound $3 t$ in $71 \%\left(72.1 \mathrm{mg}\right.$ ) as a white solid; $\mathrm{R}_{\mathrm{f}}=0.43$ (petroleum ether/ethyl acetate $\left.=20 / 1\right)$; $\mathrm{mp}$ : 68.7-69.1 ${ }^{\circ} \mathrm{C} ;{ }^{1} \mathrm{H}$ NMR (400 MHz, $\left.\mathrm{CDCl}_{3}\right) \delta 8.53(\mathrm{~s}, 1 \mathrm{H}), 8.03(\mathrm{db}, J=8.7 \mathrm{~Hz}, 1 \mathrm{H}), 7.88(\mathrm{~d}, J=9.0 \mathrm{~Hz}$, $1 \mathrm{H}), 7.81(\mathrm{~d}, J=8.8 \mathrm{~Hz}, 1 \mathrm{H}), 7.24(\mathrm{dd}, J=9.0,2.5 \mathrm{~Hz}, 1 \mathrm{H}), 7.16(\mathrm{~d}, J=2.4 \mathrm{~Hz}, 1 \mathrm{H}), 3.97(\mathrm{~s}, 3 \mathrm{H}) .{ }^{13} \mathrm{C}$ $\operatorname{NMR}\left(100 \mathrm{MHz}, \mathrm{CDCl}_{3}\right) \delta 180.1(\mathrm{q}, J=34.5 \mathrm{~Hz}), 161.1,138.5,133.0(\mathrm{q}, J=2.6 \mathrm{~Hz}), 131.9,127.7$, $127.5,125.1(\mathrm{q}, J=1.6 \mathrm{~Hz}), 120.4,117.0(\mathrm{q}, J=289.8 \mathrm{~Hz}), 105.9,55.6 .{ }^{19} \mathrm{~F} \mathrm{NMR}\left(376 \mathrm{MHz}, \mathrm{CDCl}_{3}\right) \delta$ -70.56. This compound is known. ${ }^{3}$ 
<smiles>O=C(c1ccc2cc(Br)ccc2c1)C(F)(F)F</smiles>

The representative general procedure mentioned above was followed. Purification by preparative thin-layer chromatography (PTLC) on silica gel (petroleum ether/dichloromethane $=5 / 1$ ) yielded the title compound $3 \mathbf{u}$ in $84 \%$ (101.8 $\mathrm{mg}$ ) as a white solid; $\mathrm{R}_{\mathrm{f}}=0.32$ (petroleum ether/dichloromethane $=$ 5/1); mp: 97.0-97.1 ${ }^{\circ} \mathrm{C} ;{ }^{1} \mathrm{H}$ NMR $\left(400 \mathrm{MHz}, \mathrm{CDCl}_{3}\right) \delta 8.58(\mathrm{~s}, 1 \mathrm{H}), 8.11-8.09(\mathrm{~m}, 2 \mathrm{H})$, 7.90-7.86 (m, 2H), $7.70(\mathrm{dd}, J=8.8,1.9 \mathrm{~Hz}, 1 \mathrm{H}) .{ }^{13} \mathrm{C} \mathrm{NMR}\left(100 \mathrm{MHz}, \mathrm{CDCl}_{3}\right) \delta 180.2(\mathrm{q}, J=35.2 \mathrm{~Hz}), 137.3,133.0$ $(\mathrm{q}, J=2.8 \mathrm{~Hz}), 131.6,131.1,130.6,130.2,128.2,127.5,125.4,124.8,116.8(\mathrm{q}, J=289.5 \mathrm{~Hz}) .{ }^{19} \mathrm{~F}$ NMR $\left(376 \mathrm{MHz}, \mathrm{CDCl}_{3}\right) \delta$-70.82. HRMS (ESI-TOF) m/z: [M] Calcd for $\mathrm{C}_{12} \mathrm{H}_{6} \mathrm{~F}_{3} \mathrm{BrO} 301.9554$; Found 301.9570 for the compounds: $3 \mathbf{u}$.

\section{2,2,2-trifluoro-1-(quinolin-6-yl)ethan-1-one（3v）}<smiles>O=C(c1ccc2ncccc2c1)C(F)(F)F</smiles>

The representative general procedure mentioned above was followed. Purification by preparative thin-layer chromatography (PTLC) on silica gel (petroleum ether/ethyl acetate $=1 / 2$ ) yielded the title compound $3 \mathrm{v}$ in $78 \%$ (70.2 $\mathrm{mg}$ ) as a light yellow solid; $\mathrm{R}_{\mathrm{f}}=0.56$ (petroleum ether/ethyl acetate $=1 / 2$ ); mp: 105.0-106.1 ${ }^{\circ} \mathrm{C} ;{ }^{1} \mathrm{H}$ NMR $\left(400 \mathrm{MHz}, \mathrm{CDCl}_{3}\right) \delta 9.08(\mathrm{dd}, J=4.2,1.7 \mathrm{~Hz}, 1 \mathrm{H}), 8.61(\mathrm{~s}, 1 \mathrm{H})$, $8.40-8.28(\mathrm{~m}, 2 \mathrm{H}), 8.23(\mathrm{~d}, J=9.0 \mathrm{~Hz}, 1 \mathrm{H}), 7.55(\mathrm{dd}, J=8.3,4.3 \mathrm{~Hz}, 1 \mathrm{H}) .{ }^{13} \mathrm{C} \mathrm{NMR}\left(100 \mathrm{MHz}, \mathrm{CDCl}_{3}\right)$ $\delta 180.0(\mathrm{q}, J=35.1 \mathrm{~Hz}), 154.0,150.8,138.0,130.0(\mathrm{q}, J=2.7 \mathrm{~Hz}), 130.9,128.0(\mathrm{q}, J=1.2 \mathrm{~Hz}), 127.7$, 127.3, 122.6, 116.7 (q, $J=289.5 \mathrm{~Hz}$ ). ${ }^{19} \mathrm{~F}$ NMR (376 MHz, $\left.\mathrm{CDCl}_{3}\right) \delta-70.96$. HRMS (ESI-TOF) m/z: [M] Calcd for $\mathrm{C}_{11} \mathrm{H}_{6} \mathrm{~F}_{3} \mathrm{NO} 225.0401$; Found 225.0413 for the compounds: $3 \mathbf{v}$.

\section{2,2,2-trifluoro-1-(quinolin-2-yl)ethan-1-one (3w)}<smiles>O=C(c1ccc2ccccc2n1)C(F)(F)F</smiles>

$3 w$

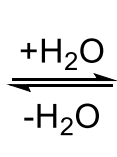<smiles>OC(O)(c1ccc2ccccc2n1)C(F)(F)F</smiles>

$3 w^{\prime}$

The representative general procedure mentioned above was followed. Purification by preparative thin-layer chromatography (PTLC) on silica gel (petroleum ether/ethyl acetate $=5 / 1$ ) yielded the title compound $3 \mathbf{w}$ in $70 \%\left(63.0 \mathrm{mg}\right.$ ) as a white solid; $\mathrm{R}_{\mathrm{f}}=0.32$ (petroleum ether/ethyl acetate $\left.=5 / 1\right)$; mp: 105.7-106.9 ${ }^{\circ} \mathrm{C}$; It is a mixture of $\mathbf{3 w}$ and its hydration 3w'. ${ }^{1} \mathrm{H}$ NMR $\left(400 \mathrm{MHz}, \mathrm{CDCl}_{3}\right) \delta 8.36-8.32$ $(\mathrm{m}, 1 \mathrm{H}), 8.13-8.11(\mathrm{~m}, 1 \mathrm{H}), 7.92-7.90(\mathrm{~m}, 1 \mathrm{H}), 7.87-7.84(\mathrm{~m}, 1 \mathrm{H}), 7.82-7.78(\mathrm{~m}, 1 \mathrm{H}), 7.67-7.63(\mathrm{~m}$, $1 \mathrm{H}), 5.71(\mathrm{~s}, 2 \mathrm{H}) .{ }^{13} \mathrm{C} \mathrm{NMR}\left(100 \mathrm{MHz}, \mathrm{CDCl}_{3}\right) \delta 152.4,145.3,138.6,130.6,129.0,128.7,128.0,127.7$, $122.8(\mathrm{q}, J=285.7 \mathrm{~Hz}) 118.8(\mathrm{q}, J=1.9 \mathrm{~Hz}), 92.1(\mathrm{q}, J=33.2 \mathrm{~Hz}) .{ }^{19} \mathrm{~F}$ NMR $\left(376 \mathrm{MHz}, \mathrm{CDCl}_{3}\right)$ $\delta-71,14,-83.47$. This compound is known. ${ }^{5}$

\section{2,2,2-trifluoro-1-(pyridin-3-yl)ethan-1-one (3x)}


$\overbrace{\mathrm{N}}^{\mathrm{O}} \mathrm{CF}_{3}$

The product $3 \mathbf{x}$ was not able to be isolated due to its volatility. As such ${ }^{1} \mathrm{H}$ NMR, ${ }^{13} \mathrm{C} \mathrm{NMR}$, and ${ }^{19} \mathrm{~F}$ NMR were not obtained. (97\% ${ }^{19} \mathrm{~F}$ NMR yield using 4-fluorobenzoic acid as an internal standard.)

\section{1-(benzofuran-2-yl)-2,2,2-trifluoroethan-1-one (3y)}<smiles>O=C(c1cc2ccccc2o1)C(F)(F)F</smiles>

The representative general procedure mentioned above was followed. Purification by preparative thin-layer chromatography (PTLC) on silica gel (petroleum ether/ethyl acetate $=5 / 1$ ) yielded the title compound $3 \mathbf{y}$ in $64 \%\left(54.8 \mathrm{mg}\right.$ ) as a yellow solid; $\mathrm{R}_{\mathrm{f}}=0.58$ (petroleum ether/ethyl acetate $\left.=5 / 1\right)$; mp: 46.0-47.4 ${ }^{\circ} \mathrm{C} ;{ }^{1} \mathrm{H}$ NMR (400 MHz, $\left.\mathrm{CDCl}_{3}\right) \delta 7.86-7.79(\mathrm{~m}, 1 \mathrm{H}), 7.80(\mathrm{~d}, J=8.0 \mathrm{~Hz}, 1 \mathrm{H}), 7.66-7.58(\mathrm{~m}$, 2H), 7.42-7.38 (m, 1H). $\left.{ }^{13} \mathrm{C} \mathrm{NMR} \mathrm{(100} \mathrm{MHz,} \mathrm{CDCl}_{3}\right) \delta 170.7$ (q, $\left.J=37.6 \mathrm{~Hz}\right), 156.8,146.5,130.6$, 126.4, 125.0, 124.2, 120.2 (q, $J=2.9 \mathrm{~Hz}), 116.2$ (q, $J=287.5 \mathrm{~Hz}), 112.8 .{ }^{19} \mathrm{~F} \mathrm{NMR}\left(376 \mathrm{MHz}, \mathrm{CDCl}_{3}\right)$ $\delta$-73.11. This compound is known. ${ }^{7}$

\section{1-(benzo[b]thiophen-2-yl)-2,2,2-trifluoroethan-1-one (3z)}<smiles>O=C(c1cc2ccccc2s1)C(F)(F)F</smiles>

The representative general procedure mentioned above was followed. Purification by preparative thin-layer chromatography (PTLC) on silica gel (petroleum ether/ethyl acetate $=100 / 1$ ) yielded the title compound $3 \mathbf{z}$ in $68 \%(62.6 \mathrm{mg})$ as a light yellow solid; $\mathrm{R}_{\mathrm{f}}=0.28$ (petroleum ether/ethyl acetate = 100/1); mp: 47.3-48.5 ${ }^{\circ} \mathrm{C}$; ${ }^{1} \mathrm{H}$ NMR (400 MHz, $\left.\mathrm{CDCl}_{3}\right) \delta 8.24$ (s, $\left.1 \mathrm{H}\right), 7.98(\mathrm{~d}, J=8.1 \mathrm{~Hz}, 1 \mathrm{H}), 7.92$ $(\mathrm{dd}, J=8.2,0.6 \mathrm{~Hz}, 1 \mathrm{H}), 7.59-7.55(\mathrm{~m}, 1 \mathrm{H}), 7.50-7.46(\mathrm{~m}, 1 \mathrm{H}) .{ }^{13} \mathrm{C} \mathrm{NMR}\left(100 \mathrm{MHz}, \mathrm{CDCl}_{3}\right) \delta 175.3$ $(\mathrm{q}, J=37.2 \mathrm{~Hz}), 143.6,138.8,135.6,134.4(\mathrm{q}, J=3.3 \mathrm{~Hz}), 129.3,127.1,125.8,123.0,116.4(\mathrm{q}, J=$ $290.4 \mathrm{~Hz}) .{ }^{19} \mathrm{~F} \mathrm{NMR}\left(376 \mathrm{MHz}, \mathrm{CDCl}_{3}\right) \delta-71.79$. This compound is known. ${ }^{6}$

\section{C(Z)-1,1,1-trifluorononadec-10-en-2-one (3aa)}<smiles>CCCCCCCCCCCC(=O)C(F)(F)F</smiles>

The representative general procedure mentioned above was followed. Purification by preparative thin-layer chromatography (PTLC) on silica gel (petroleum ether/ethyl acetate $=20 / 1$ ) yielded the title compound 3aa in $44 \%\left(58.9 \mathrm{mg}\right.$ ) as a yellow oil; $\mathrm{R}_{\mathrm{f}}=0.53$ (petroleum ether/ethyl acetate $\left.=20 / 1\right) ;{ }^{1} \mathrm{H}$ NMR (400 MHz, $\left.\mathrm{CDCl}_{3}\right) \delta 5.39-5.30(\mathrm{~m}, 2 \mathrm{H}), 2.70(\mathrm{t}, J=7.2 \mathrm{~Hz}, 1 \mathrm{H}), 2.38(\mathrm{t}, J=7.5 \mathrm{~Hz}, 1 \mathrm{H})$, 2.05-1.98 (m, 4H), 1.69-1.64 (m, 1H), 1.57-1.54 (m, 1H), 1.31-1.27 (m, 20H), $0.88(\mathrm{t}, J=6.8 \mathrm{~Hz}, 3 \mathrm{H})$. ${ }^{13} \mathrm{C}$ NMR $\left(100 \mathrm{MHz}, \mathrm{CDCl}_{3}\right) \delta 190.6(\mathrm{q}, J=3.5 \mathrm{~Hz}), 129.1,128.6,114.6(\mathrm{q}, J=290.5 \mathrm{~Hz}), 35.3,30.9$, 28.7, 28.6, 28.5, 28.3, 28.1, 28.0, 27.7, 26.2, 26.1, 21.7, 21.3, 13.1. ${ }^{19} \mathrm{~F} \mathrm{NMR}\left(376 \mathrm{MHz}, \mathrm{CDCl}_{3}\right) \delta$ -79.35. HRMS (ESI-TOF) m/z: [M-H] $]^{-}$Calcd for $\mathrm{C}_{19} \mathrm{H}_{33} \mathrm{~F}_{3} \mathrm{O}-\mathrm{H}$ 333.2411; Found 333.2411 for the compounds: 3aa. 


\section{1,1,1-trifluorononadecan-2-one (3ab)}<smiles>CCCCCCCCCCCCC(=O)C(F)(F)F</smiles>

The representative general procedure mentioned above was followed. Purification by preparative thin-layer chromatography (PTLC) on silica gel (petroleum ether/ethyl acetate $=20 / 1$ ) yielded the title compound 3ab in $43 \%\left(57.8 \mathrm{mg}\right.$ ) as a white solid; $\mathrm{R}_{\mathrm{f}}=0.57$ (petroleum ether/ethyl acetate $\left.=20 / 1\right)$. $\mathrm{mp}$ : $79.5-80.6{ }^{\circ} \mathrm{C} ;{ }^{1} \mathrm{H}$ NMR $\left(400 \mathrm{MHz}, \mathrm{CDCl}_{3}\right) \delta 2.70(\mathrm{t}, J=7.2 \mathrm{~Hz}, 2 \mathrm{H}), 1.71-1.63(\mathrm{~m}, 2 \mathrm{H}), 1.33-1.25(\mathrm{~m}$, $28 \mathrm{H}), 0.88(\mathrm{t}, J=6.8 \mathrm{~Hz}, 3 \mathrm{H}) .{ }^{13} \mathrm{C} \mathrm{NMR}\left(100 \mathrm{MHz}, \mathrm{CDCl}_{3}\right) \delta 191.7(\mathrm{q}, J=34.5 \mathrm{~Hz}), 115.6(\mathrm{q}, J=$ $290.4 \mathrm{~Hz}), 36.4,31.9,29.7,29.8,29.7,29.6,29.5,29.4,29.4,29.2,28.8,22.7,22.4,14.1 .{ }^{19} \mathrm{~F} \mathrm{NMR}(376$ $\left.\mathrm{MHz}, \mathrm{CDCl}_{3}\right) \delta-79.35$. HRMS (ESI-TOF) m/z: [M-H] $]^{-}$Calcd for $\mathrm{C}_{19} \mathrm{H}_{35} \mathrm{~F}_{3} \mathrm{O}-\mathrm{H}$ 335.2567; Found 335.2562 for the compounds: 3ab.

1-(6-(3-((3r,5r,7r)-adamantan-1-yl)-4-methoxyphenyl)naphthalen-2-yl)-2,2,2-trifluoroethan-1-one (3ac)<smiles>COc1ccc(-c2ccc3cc(C(=O)C(F)(F)F)ccc3c2)cc1C12CC3CC(CC(C3)C1)C2</smiles>

The representative general procedure mentioned above was followed. Purification by preparative thin-layer chromatography (PTLC) on silica gel (petroleum ether/ethyl acetate $=40 / 1$ ) yielded the title compound 3ac in $80 \%(148.5 \mathrm{mg})$ as a yellow solid; $\mathrm{R}_{\mathrm{f}}=0.31$ (petroleum ether/ethyl acetate $\left.=40 / 1\right)$; mp:191.5-193.4 ${ }^{\circ} \mathrm{C} ;{ }^{1} \mathrm{H}$ NMR (400 MHz, $\left.\mathrm{CDCl}_{3}\right) \delta 8.63(\mathrm{~s}, 1 \mathrm{H}), 8.10-7.98(\mathrm{~m}, 4 \mathrm{H}), 7.87$ (dd, $J=8.6$, $1.6 \mathrm{~Hz}, 1 \mathrm{H}), 7.63(\mathrm{~d}, J=2.3 \mathrm{~Hz}, 1 \mathrm{H}), 7.57(\mathrm{dd}, J=8.4,2.3 \mathrm{~Hz}, 1 \mathrm{H}), 7.02(\mathrm{~d}, J=8.5 \mathrm{~Hz}, 1 \mathrm{H}), 3.92(\mathrm{~s}$, $3 \mathrm{H}), 2.20(\mathrm{~b}, 3 \mathrm{H}), 2.13(\mathrm{~b}, 3 \mathrm{H}), 1.82(\mathrm{~b}, 6 \mathrm{H}) .{ }^{13} \mathrm{C} \mathrm{NMR}\left(100 \mathrm{MHz}, \mathrm{CDCl}_{3}\right) \delta 180.3$ (q, J = 34.8 Hz), 159.3, 143.3, 139.2, 137.0, 133.0 (q, $J=2.5$ Hz), 132.0, 130.9, 130.6, 129.1, 127.2, 126.7, 126.0, 126.9, $124.7,124.6,117.0(\mathrm{q}, J=289.7 \mathrm{~Hz}), 112.2,55.2,40.6,37.3,37.1,29.1 .{ }^{19} \mathrm{~F} \mathrm{NMR}\left(376 \mathrm{MHz}, \mathrm{CDCl}_{3}\right) \delta$ -70.59. HRMS (ESI-TOF) m/z: [M] Calcd for $\mathrm{C}_{29} \mathrm{H}_{27} \mathrm{~F}_{3} \mathrm{O}_{2}$ 464.1963; Found 464.1963 for the compounds: 3ac.

\section{N,N-dipropyl-4-(2,2,2-trifluoroacetyl)benzenesulfonamide (3ad)}<smiles>CCCN(CCC)S(=O)(=O)c1ccc(C(=O)C(F)(F)F)cc1</smiles>

The representative general procedure mentioned above was followed. Purification by preparative 
thin-layer chromatography (PTLC) on silica gel (petroleum ether/ethyl acetate $=3 / 1$ ) yielded the title compound 3ad in $90 \%(121.3 \mathrm{mg})$ as a light yellow solid; $\mathrm{R}_{\mathrm{f}}=0.28$ (petroleum ether/ethyl acetate $=$ 3/1); mp: 68.9-70.2 ${ }^{\circ} \mathrm{C} ;{ }^{1} \mathrm{H}$ NMR $\left(400 \mathrm{MHz}, \mathrm{CDCl}_{3}\right) \delta 8.18(\mathrm{~d}, J=8.0 \mathrm{~Hz}, 2 \mathrm{H}), 7.99-7.96(\mathrm{~m}, 2 \mathrm{H})$, 3.14-3.10 (m, 4H), 1.61-1.51 (m, 4H), 0.89-0.84 (m, 6H). ${ }^{13} \mathrm{C} \mathrm{NMR}\left(100 \mathrm{MHz}, \mathrm{CDCl}_{3}\right) \delta 179.6(\mathrm{q}, J=$ $35.9 \mathrm{~Hz}), 146.6,132.4,130.7(\mathrm{q}, J=2.0 \mathrm{~Hz}), 127.6,116.4(\mathrm{q}, J=289.0 \mathrm{~Hz}), 50.0,22.0,11.1 .{ }^{19} \mathrm{~F} \mathrm{NMR}$ $\left(376 \mathrm{MHz}, \mathrm{CDCl}_{3}\right) \delta$-71.72. HRMS (ESI-TOF) $\mathrm{m} / \mathrm{z}$ : [M] Calcd for $\mathrm{C}_{14} \mathrm{H}_{18} \mathrm{~F}_{3} \mathrm{NO}_{3} \mathrm{~S}$ 337.0959; Found 337.0957 for the compounds: 3ad.

\section{3-methyl-2-phenyl-8-(2,2,2-trifluoroacetyl)-4H-chromen-4-one (3ae)}<smiles>Cc1c(-c2ccccc2)oc2c(C(=O)C(F)(F)F)cccc2c1=O</smiles>

The representative general procedure mentioned above was followed. Purification by preparative thin-layer chromatography (PTLC) on silica gel (petroleum ether/ethyl acetate $=1 / 1$ ) yielded the title compound 3ae in $80 \%\left(106.2 \mathrm{mg}\right.$ ) as a yellow solid; $R_{\mathrm{f}}=0.57$ (petroleum ether/ethyl acetate $\left.=1 / 1\right)$; $\mathrm{mp}$ : 128.7-130.3 ${ }^{\circ} \mathrm{C} ;{ }^{1} \mathrm{H}$ NMR $\left(400 \mathrm{MHz}, \mathrm{CDCl}_{3}\right) \delta 8.59(\mathrm{dd}, J=7.9,1.7 \mathrm{~Hz}, 1 \mathrm{H}), 8.16(\mathrm{~d}, J=7.6 \mathrm{~Hz}, 1 \mathrm{H})$, 7.75-7.73 (m, 2H), 7.56-7.52 (m, 4H), $2.23(\mathrm{~s}, 3 \mathrm{H}) .{ }^{13} \mathrm{C}$ NMR (100 MHz, $\left.\mathrm{CDCl}_{3}\right) \delta 179.7(\mathrm{q}, J=36.6$ $\mathrm{Hz}), 177.6,161.3,154.7,135.5$ (q, $J=2.4 \mathrm{~Hz}), 133.2,132.3,130.8,129.3,128.6,124.3,123.5,121.1$, 118.5, $116.0(\mathrm{q}, J=289.7 \mathrm{~Hz}), 11.8 .{ }^{19} \mathrm{~F}$ NMR $\left(376 \mathrm{MHz}, \mathrm{CDCl}_{3}\right) \delta-72.75$. HRMS (ESI-TOF) m/z: [M] Calcd for $\mathrm{C}_{18} \mathrm{H}_{11} \mathrm{~F}_{3} \mathrm{O}_{3}$ 333.0660; Found 333.0660 for the compounds: 3ae.

1-(4' -((1,7'-dimethyl-2'-propyl-1H,3'H-[2,5'-bibenzo[d]imidazol]-3'-yl)methyl)-[1,1'-biphenyl]-2-yl )-2,2,2-trifluoroethan-1-one (3af)

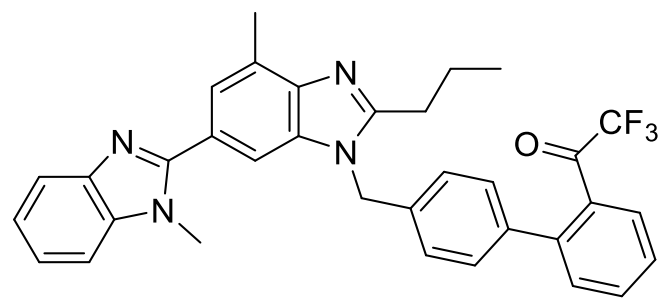

The representative general procedure mentioned above was followed. Purification by preparative thin-layer chromatography (PTLC) on silica gel (ethyl acetate) yielded the title compound 3af in 54\% $(122.3 \mathrm{mg})$ as a white solid; $\mathrm{R}_{\mathrm{f}}=0.53$ (ethyl acetate); $\mathrm{mp}: 100.5-102.2{ }^{\circ} \mathrm{C} ;{ }^{1} \mathrm{H} \mathrm{NMR}\left(400 \mathrm{MHz}, \mathrm{CDCl}_{3}\right)$ $\delta 7.82-7.78(\mathrm{~m}, 1 \mathrm{H}), 7.74(\mathrm{~d}, J=7.8 \mathrm{~Hz}, 1 \mathrm{H}), 7.62(\mathrm{td}, J=7.6,1.2 \mathrm{~Hz}, 1 \mathrm{H}), 7.50-7.45(\mathrm{~m}, 3 \mathrm{H})$, $7.41-7.33(\mathrm{~m}, 4 \mathrm{H}), 7.19(\mathrm{~d}, J=8.2 \mathrm{~Hz}, 2 \mathrm{H}), 7.10(\mathrm{~d}, J=8.2 \mathrm{~Hz}, 2 \mathrm{H}), 5.43(\mathrm{~s}, 2 \mathrm{H}), 3.77(\mathrm{~s}, 3 \mathrm{H})$, 2.94-2.90 (m, 2H), $2.77(\mathrm{~s}, 3 \mathrm{H}), 1.90-1.81(\mathrm{~m}, 2 \mathrm{H}), 1.05(\mathrm{t}, J=7.4 \mathrm{~Hz}, 3 \mathrm{H}) .{ }^{13} \mathrm{C}$ NMR (100 MHz, $\left.\mathrm{CDCl}_{3}\right) \delta 184.9(\mathrm{q}, J=35.3 \mathrm{~Hz}), 156.5,154.7,143.2,143.0,142.8,139.5,136.7,135.7,135.0,133.1$, $131.5,131.4,129.6,129.4,129.0(\mathrm{q}, J=2.2 \mathrm{~Hz}), 127.6,126.4,124.0,122.5,122.3,119.6,115.9(\mathrm{~d}, J=$ $290.7 \mathrm{~Hz}), 109.5,108.8,47.0,31.8,29.8,21.8,16.9,14.1 .{ }^{19} \mathrm{~F} \mathrm{NMR}\left(376 \mathrm{MHz}, \mathrm{CDCl}_{3}\right) \delta-72.85$. HRMS (ESI-TOF) m/z: $[\mathrm{M}+\mathrm{H}]^{+}$Calcd for $\mathrm{C}_{34} \mathrm{H}_{29} \mathrm{~F}_{3} \mathrm{~N}_{4} \mathrm{O}+\mathrm{H}$ 567.2366; Found 567.2364 for the compounds:3af. 


\section{References}

[1] Wu, W.; Tian, Q.; Chen, T.; Weng, Z. Chem. Eur. J. 2016, 22, 16455-16458.

[2] Liu, Z.; Tan, H.; Wang, L.; Fu, T.; Xia, Y.; Zhang, Y.; Wang, J. Angew. Chem. Int. Ed. 2015, 54, 3056-3060.

[3] Johansen, M. B.; Gedde, O. R.; Mayer, T. S.; Skrydstrup, T. Org. Lett. 2020, 22, 4068-4072.

[4] Rudzinski, D. M.; Kelly, C. B.; Leadbeater, N. E. Chem. Commun. 2012, 48, 9610-9612.

[5] O’Connor, M. J.; Boblak, K. N.; Topinka, M. J.; Kindelin, P. J.; Briski, J. M.; Zheng, C.; Klumpp, D. A. J. Am. Chem. Soc. 2010, 132, 3266-3267.

[6] Heinz, B.; Djukanovic, D.; Ganiek, M. A.; Martin, B.; Schenkel, B.; Knochel, P. Org. Lett. 2020, 22, 493-496.

[7] Yi, X.; Cao, Y.-F.; Wang, X.; Xu, H.; Ban, S.-R.; Dai. H.-X. Tetrahedron Lett. 2020, 61, 151780.

[8] Han, Z.; Chen, S.; Tu, Y.; Lian, X.; Li, G. Eur. J. Org. Chem. 2019, 29, 4658-4661.

[9] Zhu, F.; Yang, G.; Zhou, S.; Wu, X. -F. RSC Adv. 2016, 6, 57070-57074.

[10] Guo, X.; Li, C.-J. Org. Lett. 2011, 13, 4977-4979. 


\section{Copies of ${ }^{1} \mathrm{H},{ }^{13} \mathrm{C}$ and ${ }^{19} \mathrm{~F}$ NMR Spectra of the Products}

${ }^{1} \mathrm{H}$ NMR (400 MHz, $\mathrm{CDCl}_{3}$ ) spectrum for 3a

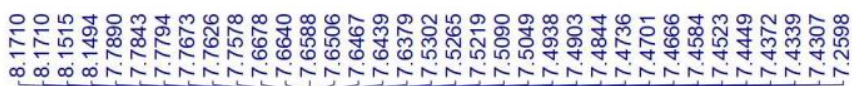
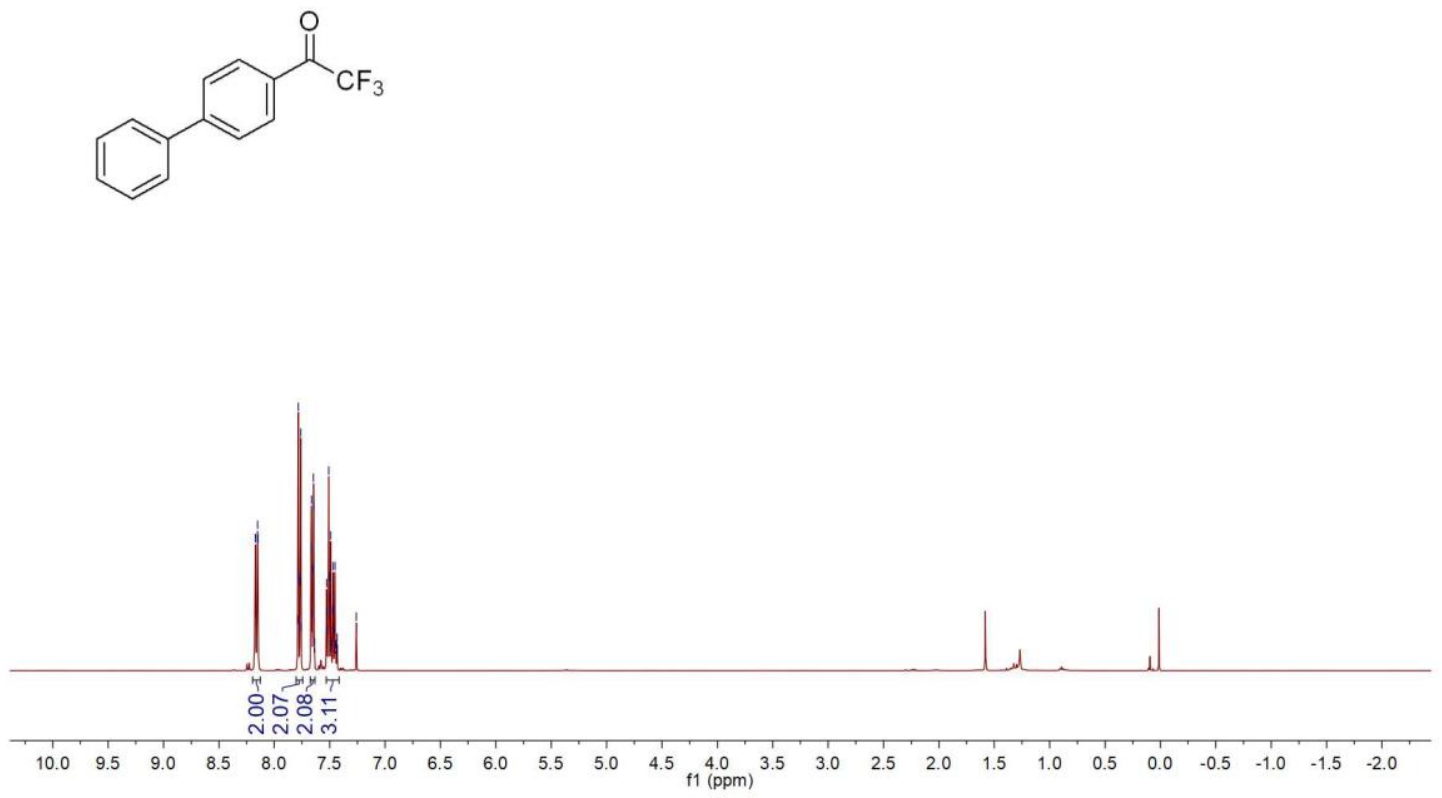

${ }^{13} \mathrm{C}$ NMR (100 MHz, $\left.\mathrm{CDCl}_{3}\right)$ spectrum for $3 \mathrm{a}$

둥.<smiles>O=C(c1ccc(-c2ccccc2)cc1)C(F)(F)F</smiles>

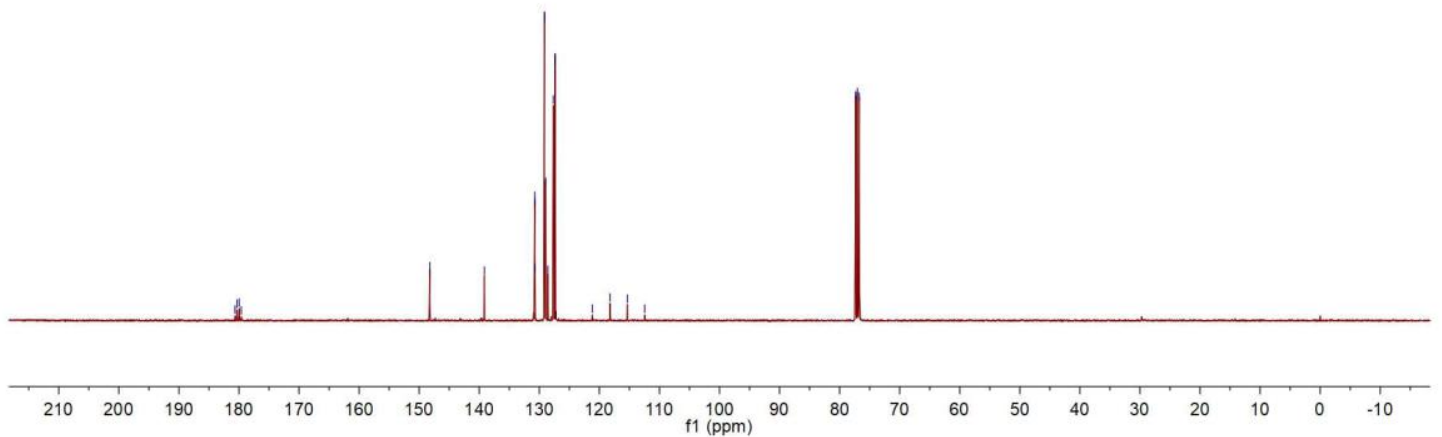


${ }^{19}$ F NMR (376 MHz, CDCl 3 ) spectrum for 3a

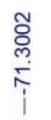<smiles>O=C(c1ccc(-c2ccccc2)cc1)C(F)(F)F</smiles>

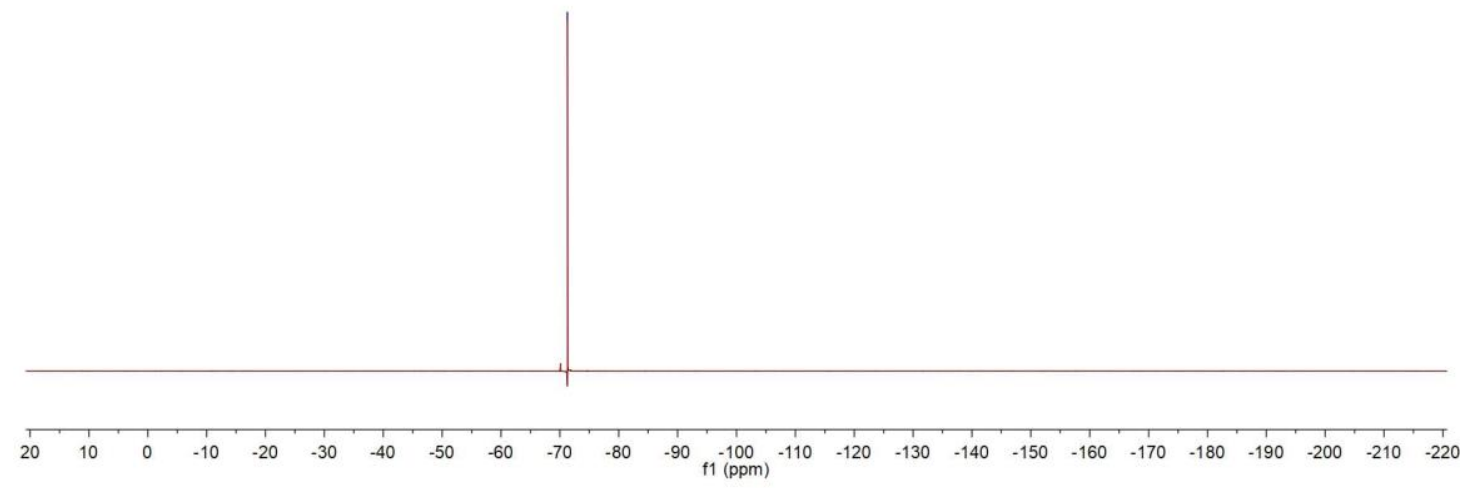

${ }^{1} \mathrm{H}$ NMR (400 MHz, $\mathrm{CDCl}_{3}$ ) spectrum for $3 \mathrm{~b}$

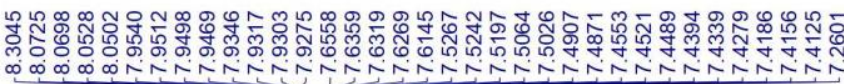<smiles>O=C(c1cccc(-c2ccccc2)c1)C(F)(F)F</smiles>

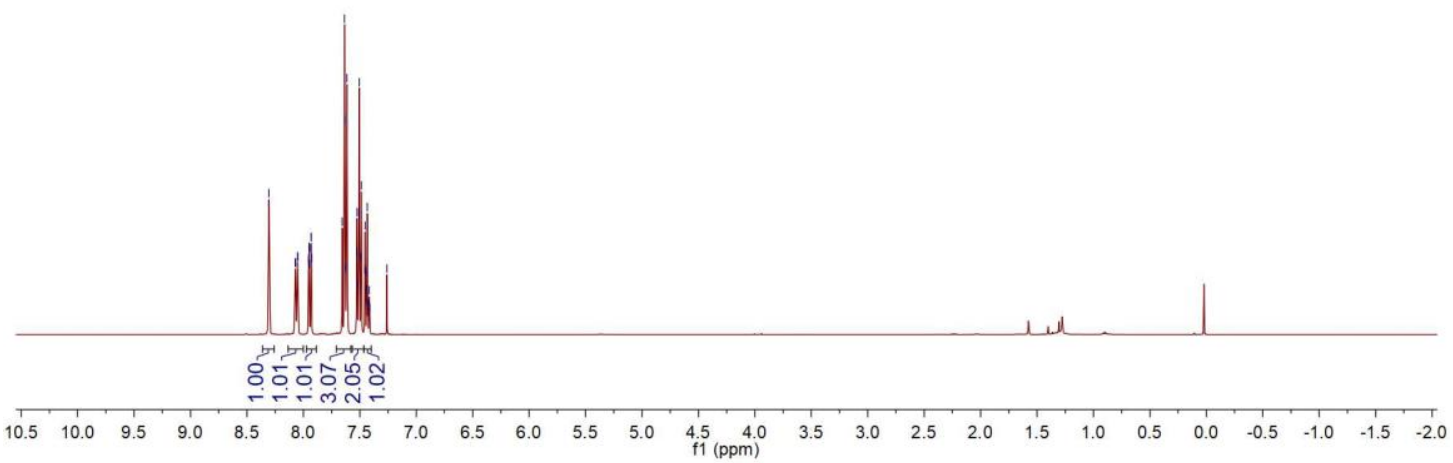


${ }^{13} \mathrm{C}$ NMR (100 MHz, $\mathrm{CDCl}_{3}$ ) spectrum for $3 \mathrm{~b}$

$\begin{array}{lll} & \\ 0 & \end{array}$
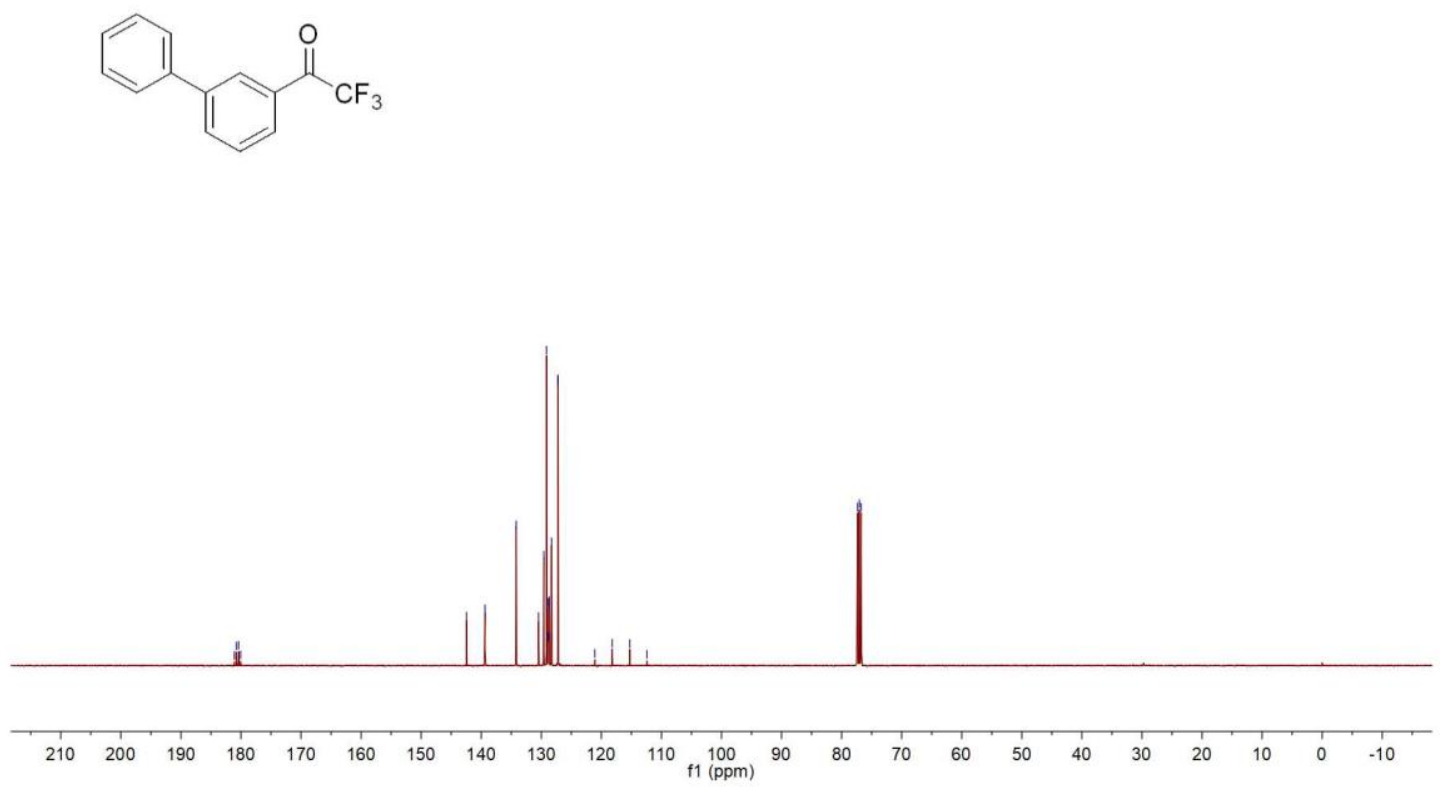

${ }^{19} \mathrm{~F}$ NMR (376 $\mathrm{MHz}, \mathrm{CDCl}_{3}$ ) spectrum for $3 \mathrm{~b}$

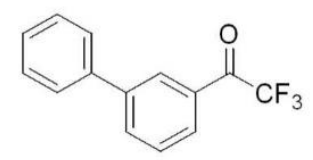

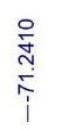

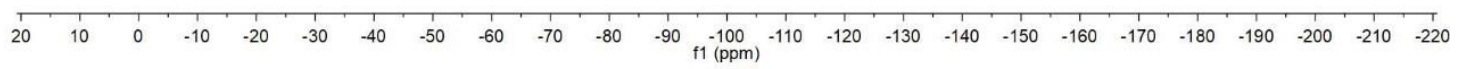

S20 
${ }^{1} \mathrm{H}$ NMR (400 MHz, $\mathrm{CDCl}_{3}$ ) spectrum for $3 \mathrm{c}$

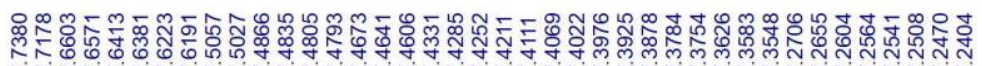

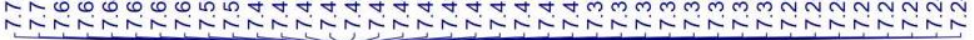<smiles>O=C(c1ccccc1-c1ccccc1)C(F)(F)F</smiles>

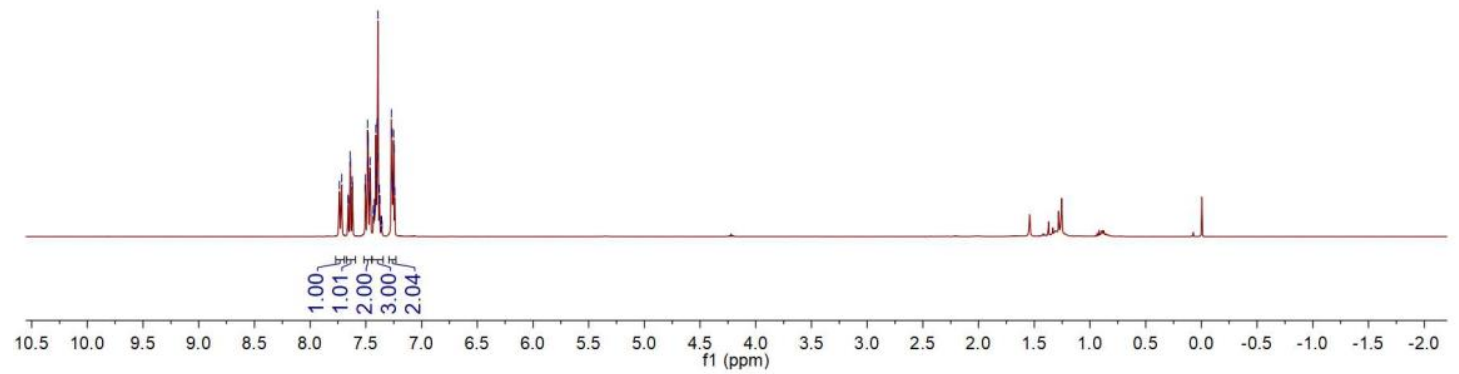

${ }^{13} \mathrm{C}$ NMR (100 MHz, $\mathrm{CDCl}_{3}$ ) spectrum for $3 \mathrm{c}$

츙앙유

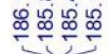

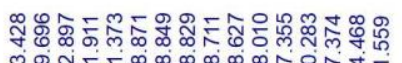

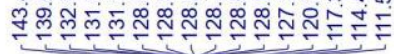

$\bar{d}$

Nîn<smiles>O=C(c1ccccc1-c1ccccc1)C(F)(F)F</smiles>

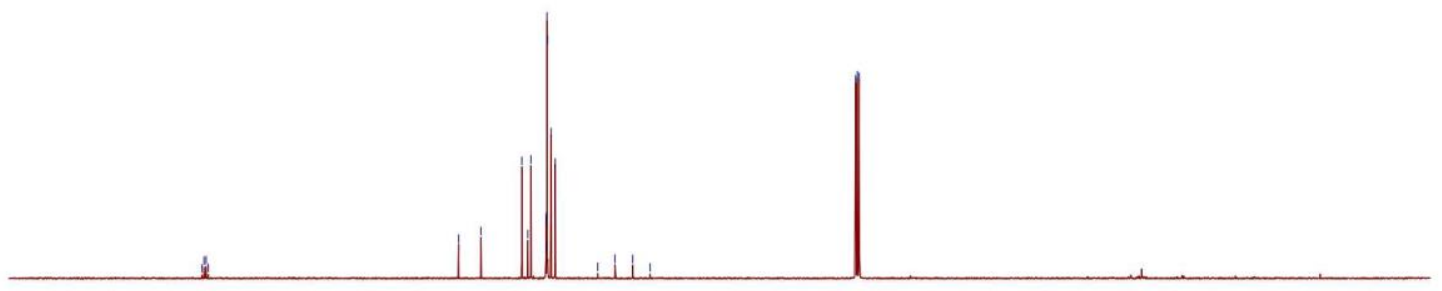

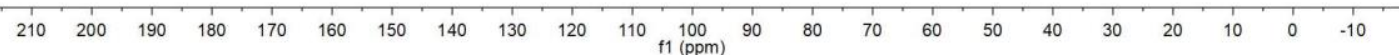


${ }^{19}$ F NMR (376 MHz, $\left.\mathrm{CDCl}_{3}\right)$ spectrum for $3 \mathrm{c}$

$$
\text { ơ }
$$<smiles>O=C(c1ccccc1-c1ccccc1)C(F)(F)F</smiles>

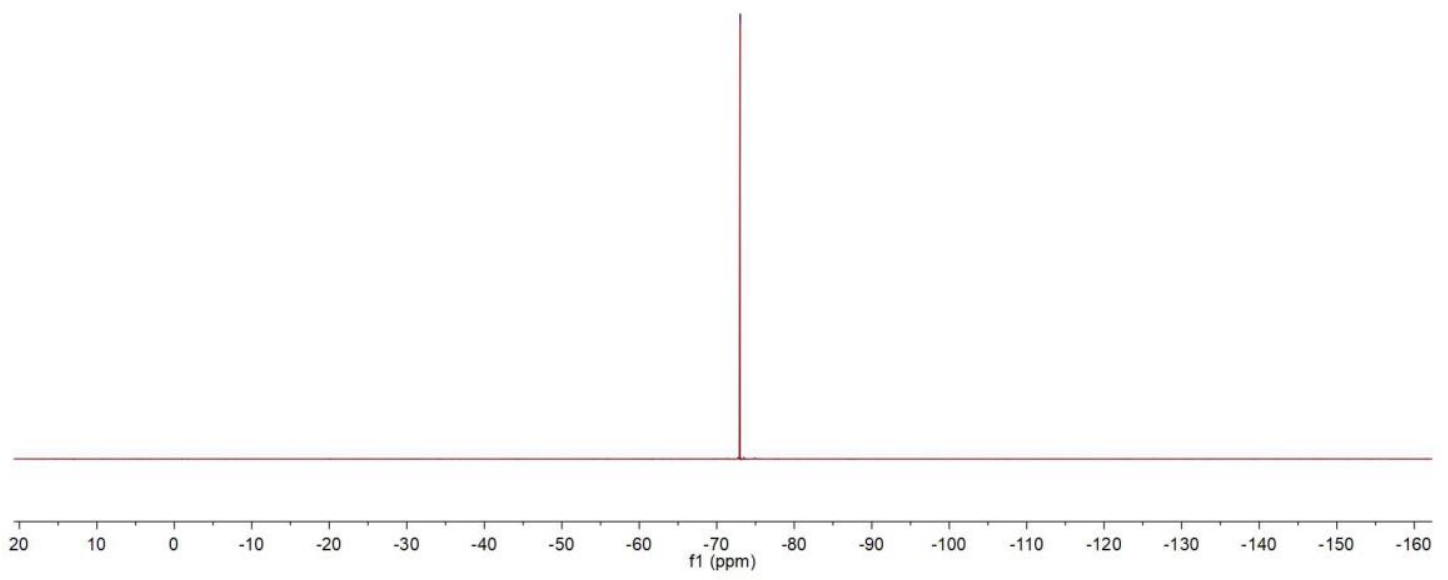

${ }^{1} \mathrm{H}$ NMR (400 MHz, $\mathrm{CDCl}_{3}$ ) spectrum for 3d 亭楊量<smiles>O=C(c1ccc(C2CCCCC2)cc1)C(F)(F)F</smiles>

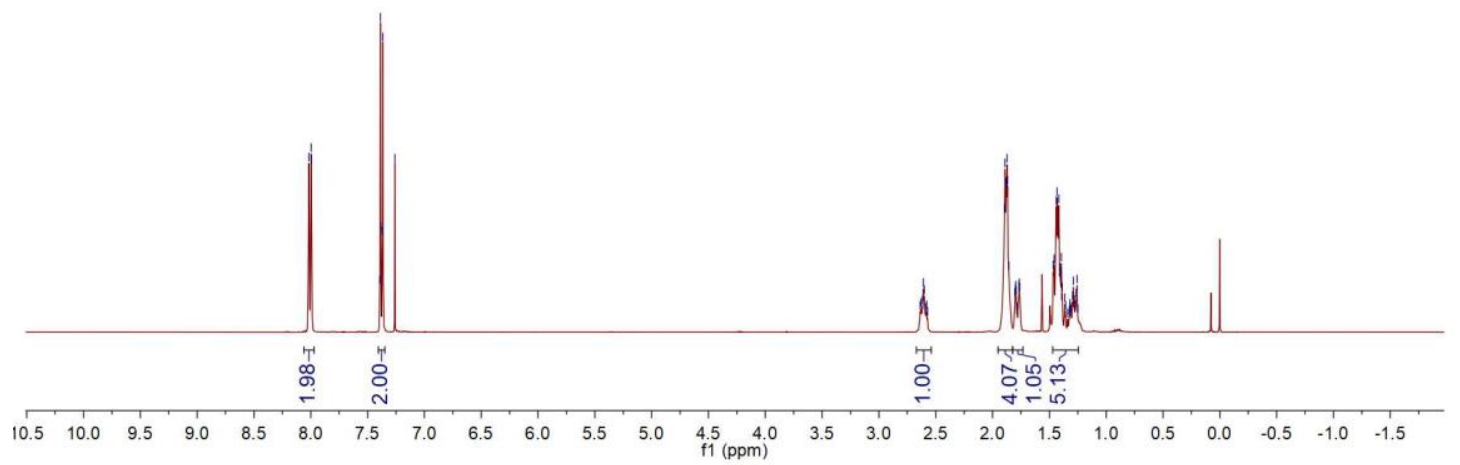


${ }^{13} \mathrm{C}$ NMR (100 MHz, $\left.\mathrm{CDCl}_{3}\right)$ spectrum for 3d

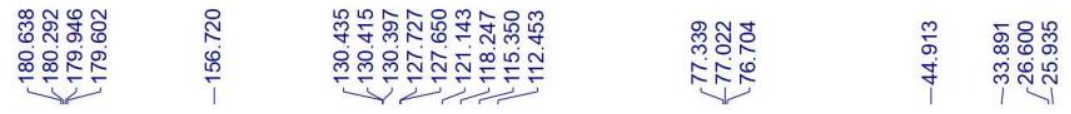<smiles>O=C(c1ccc(C2CCCCC2)cc1)C(F)(F)F</smiles>

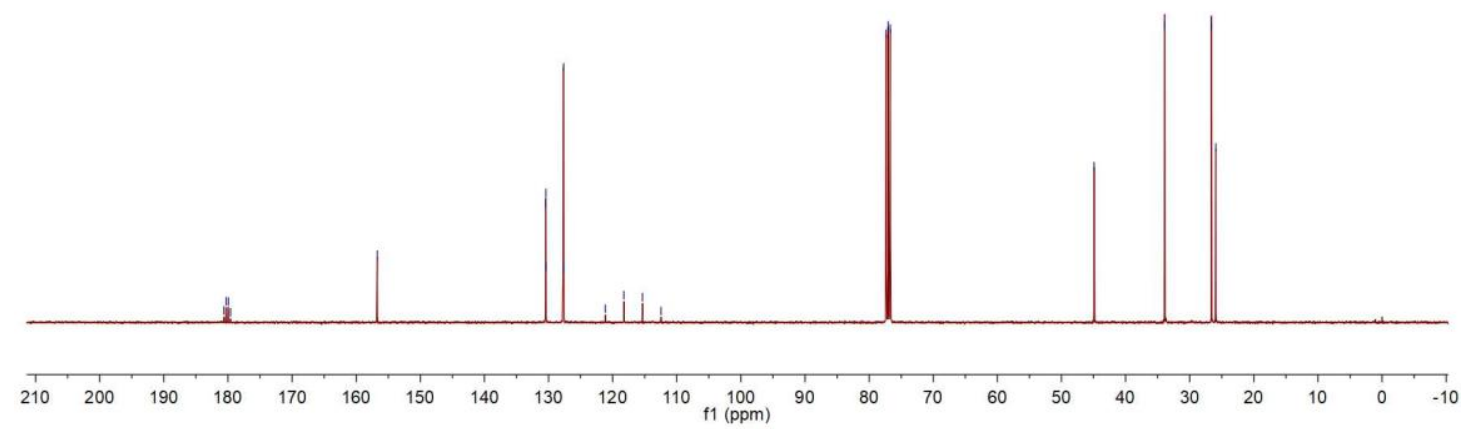

${ }^{19}$ F NMR (376 $\mathrm{MHz}, \mathrm{CDCl}_{3}$ ) spectrum for $3 \mathrm{~d}$

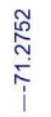<smiles>O=C(c1ccc(C2CCCCC2)cc1)C(F)(F)F</smiles>

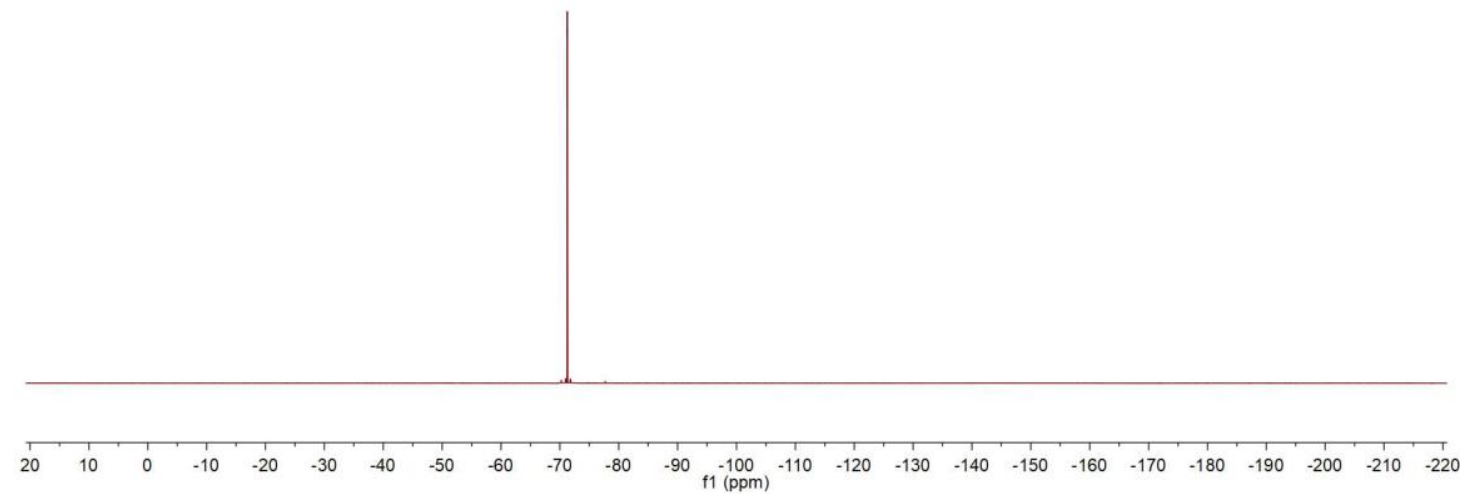


${ }^{1} \mathrm{H}$ NMR (400 MHz, $\mathrm{CDCl}_{3}$ ) spectrum for $3 \mathrm{e}$

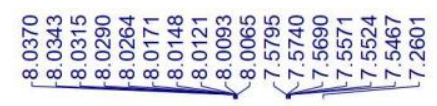<smiles>CC(C)(C)c1ccc(C(=O)C(F)(F)F)cc1</smiles>

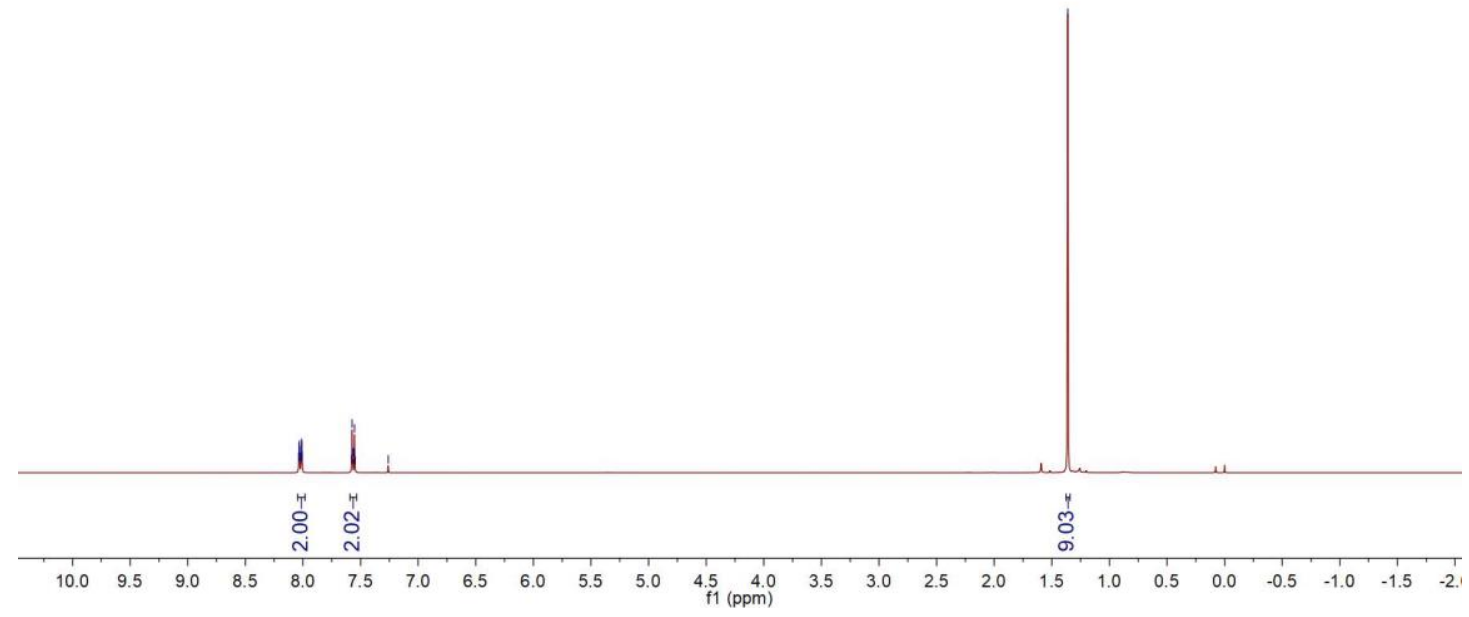

${ }^{13} \mathrm{C}$ NMR (100 MHz, $\left.\mathrm{CDCl}_{3}\right)$ spectrum for $3 \mathrm{e}$

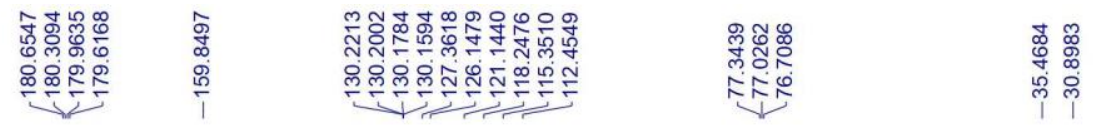<smiles>CC(C)(C)c1ccc(C(=O)C(F)(F)F)cc1</smiles>

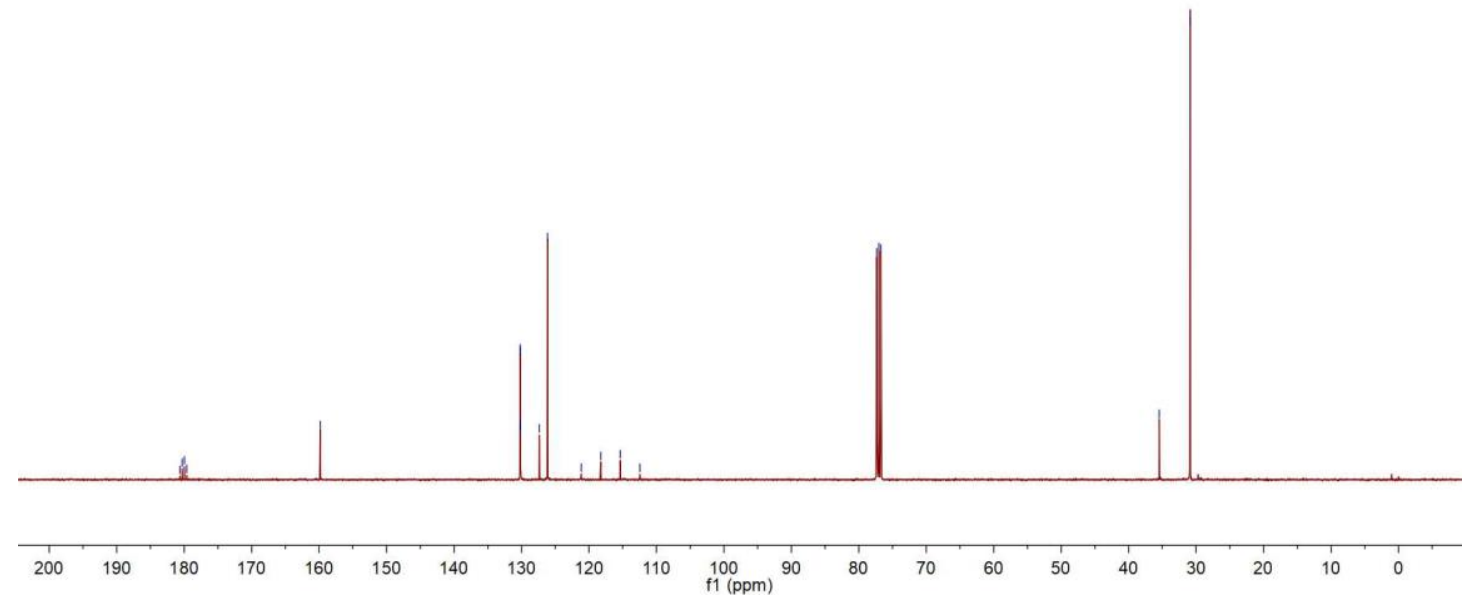


${ }^{19}$ F NMR (376 MHz, $\mathrm{CDCl}_{3}$ ) spectrum for $3 \mathrm{e}$

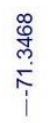<smiles>CC(C)(C)c1ccc(C(=O)C(F)(F)F)cc1</smiles>

$\begin{array}{lllllllllllllllllllllllllllllll}20 & 10 & 0 & -10 & -20 & -30 & -40 & -50 & -60 & -70 & -80 & -90 & -100 & -110 & -120 & -130 & -140 & -150 & -160 & -170 & -180 & -190 & -200 & -210 & -22\end{array}$

${ }^{1} \mathrm{H}$ NMR (400 MHz, $\mathrm{CDCl}_{3}$ ) spectrum for $3 f$

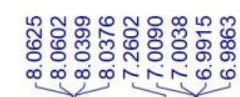

ồ<smiles>COc1ccc(C(=O)C(F)(F)F)cc1</smiles>

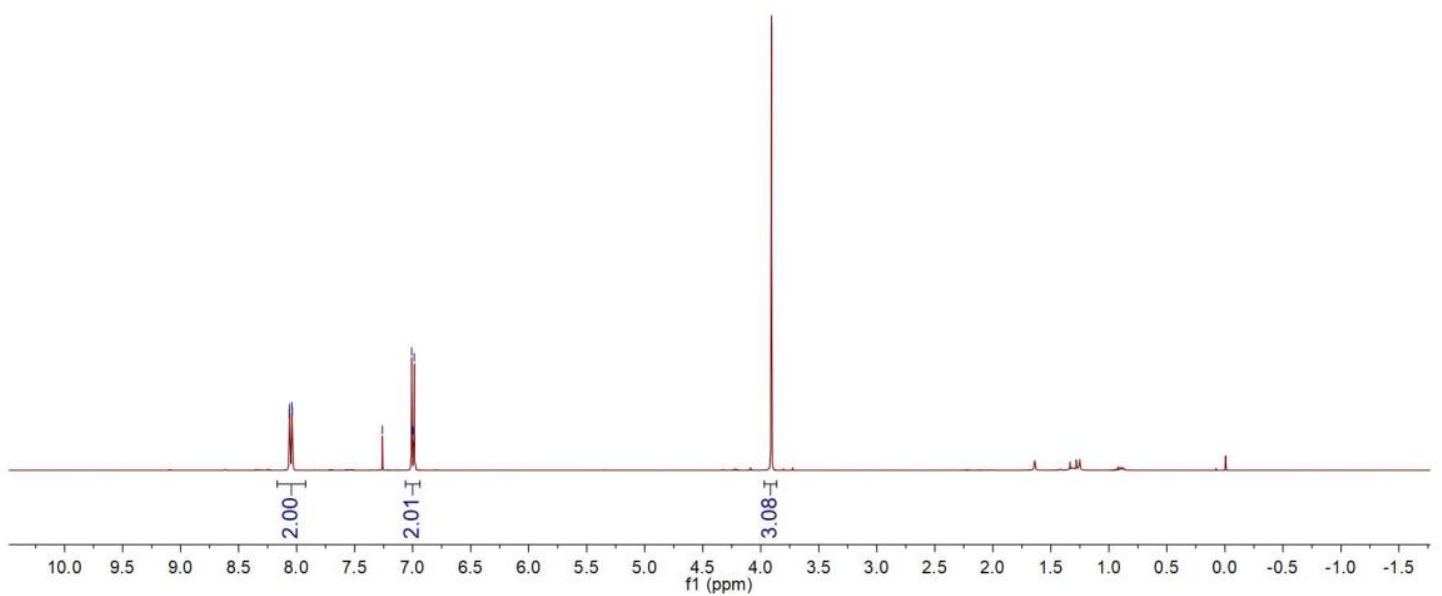


${ }^{13} \mathrm{C}$ NMR (100 MHz, $\left.\mathrm{CDCl}_{3}\right)$ spectrum for $3 f$

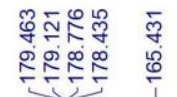

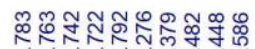

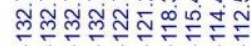

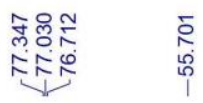<smiles>COc1ccc(C(=O)C(C)(F)F)cc1</smiles>

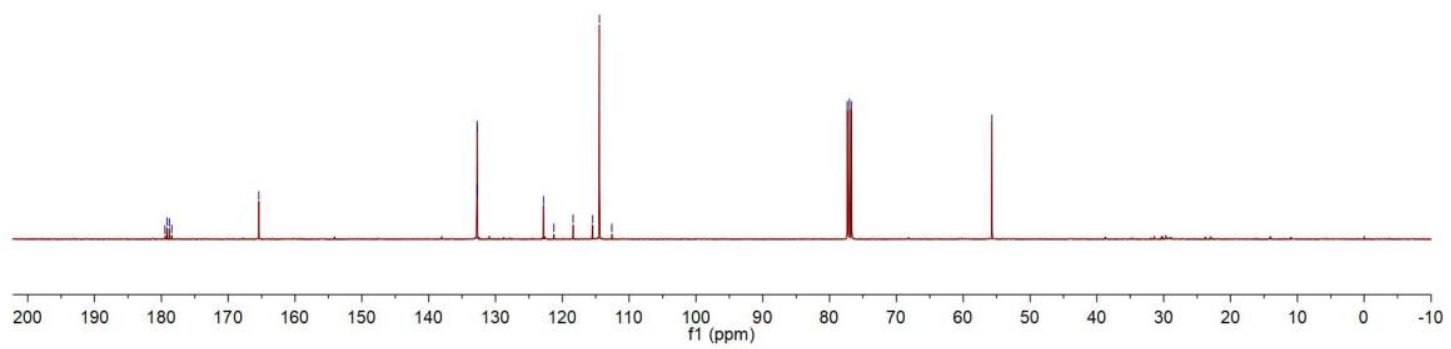

${ }^{19}$ F NMR (376 $\mathrm{MHz}, \mathrm{CDCl}_{3}$ ) spectrum for $3 \mathrm{f}$

$\stackrel{\substack{i \\ i}}{i}$<smiles>COc1ccc(C(=O)C(F)(F)F)cc1</smiles>

$\begin{array}{llllllllllllllllllllllllllllllllllllllllllll}20 & 10 & 0 & -10 & -20 & -30 & -40 & -50 & -60 & -70 & -80 & -90 & -100 & -110 & -120 & -130 & -140 & -150 & -160 & -170 & -180 & -190 & -200 & -210 & -220\end{array}$ 
${ }^{1} \mathrm{H}$ NMR (400 MHz, $\left.\mathrm{CDCl}_{3}\right)$ spectrum for 3g

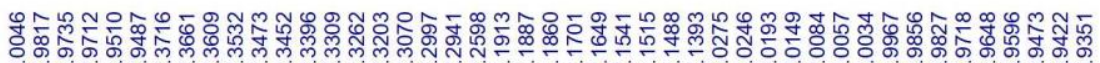

on<smiles>O=C(c1ccc(Oc2ccccc2)cc1)C(F)(F)F</smiles>

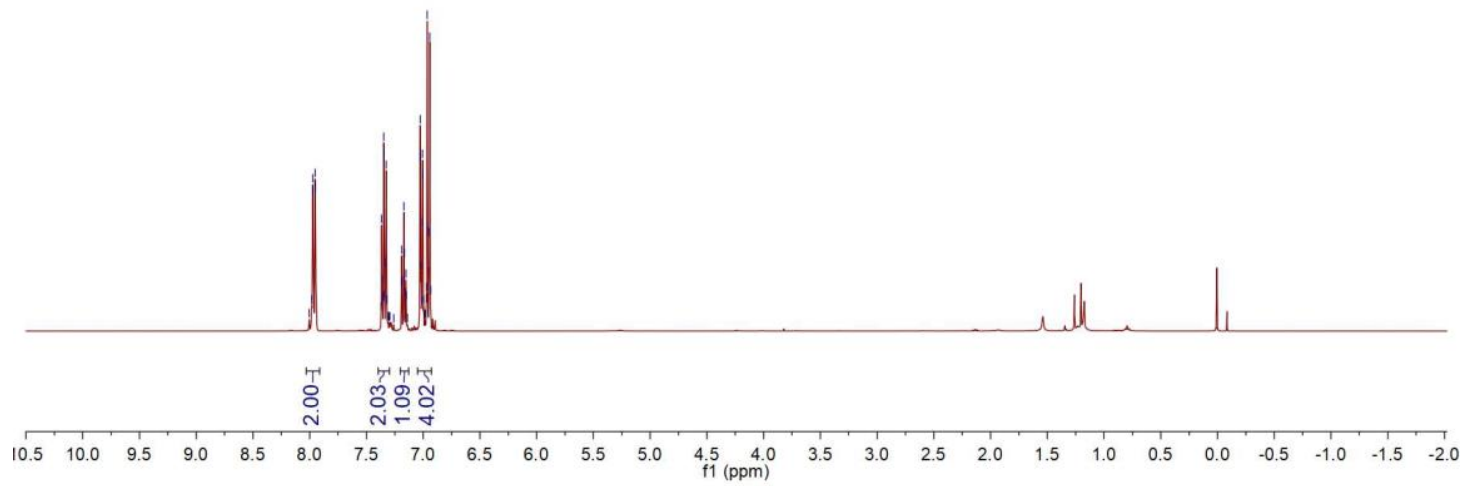

${ }^{13} \mathrm{C}$ NMR (100 MHz, $\left.\mathrm{CDCl}_{3}\right)$ spectrum for 3g

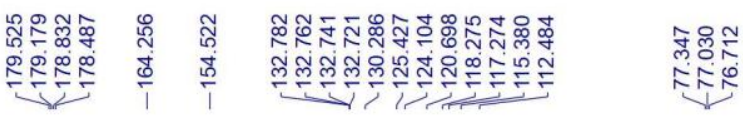<smiles>O=C(c1ccc(Oc2ccccc2)cc1)C(F)(F)F</smiles>

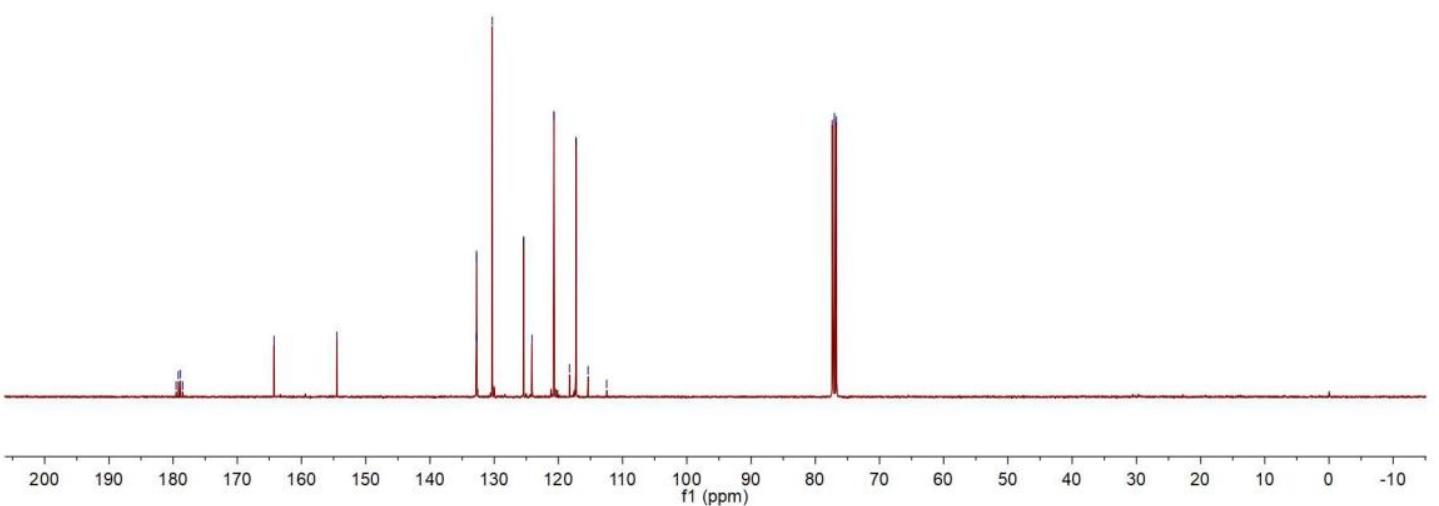


${ }^{19}$ F NMR (376 MHz, CDCl 3 ) spectrum for $3 \mathrm{~g}$

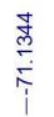<smiles>O=C(c1ccc(Oc2ccccc2)cc1)C(F)(F)F</smiles>

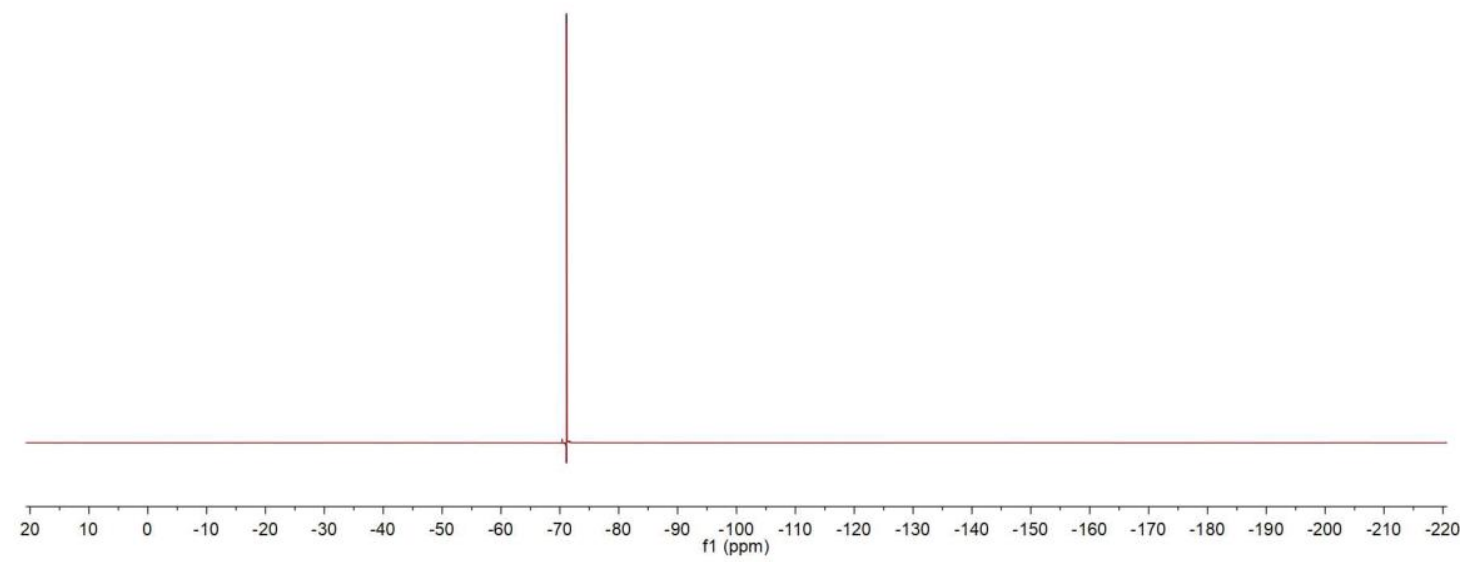

${ }^{1} \mathrm{H}$ NMR (400 MHz, $\mathrm{CDCl}_{3}$ ) spectrum for $3 \mathrm{~h}$

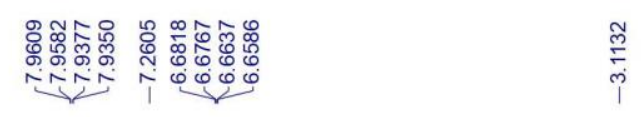<smiles>CN(C)c1ccc(C(=O)C(F)(F)F)cc1</smiles>

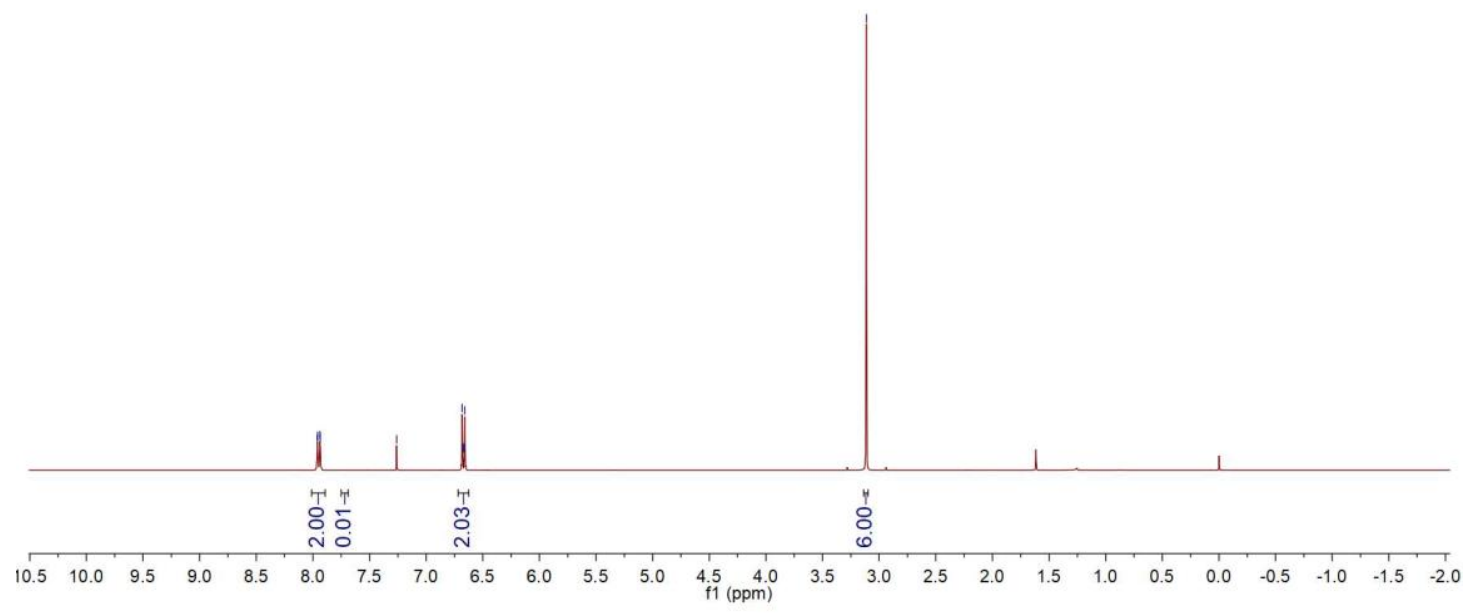


${ }^{13} \mathrm{C}$ NMR (100 MHz, $\left.\mathrm{CDCl}_{3}\right)$ spectrum for $3 \mathrm{~h}$

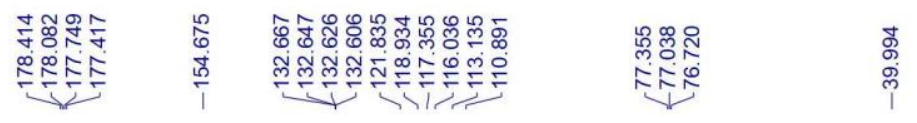<smiles>CN(C)c1ccc(C(=O)C(F)(F)F)cc1</smiles>

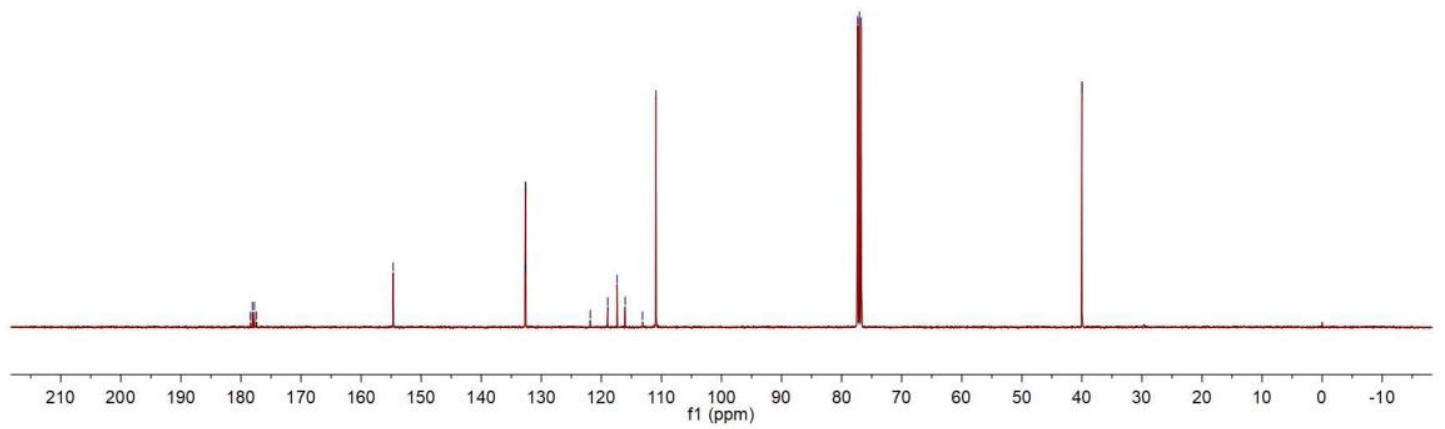

${ }^{19}$ F NMR (376 $\mathrm{MHz}, \mathrm{CDCl}_{3}$ ) spectrum for $3 \mathrm{~h}$<smiles>CN(C)c1ccc(C(=O)C(F)(F)F)cc1</smiles>

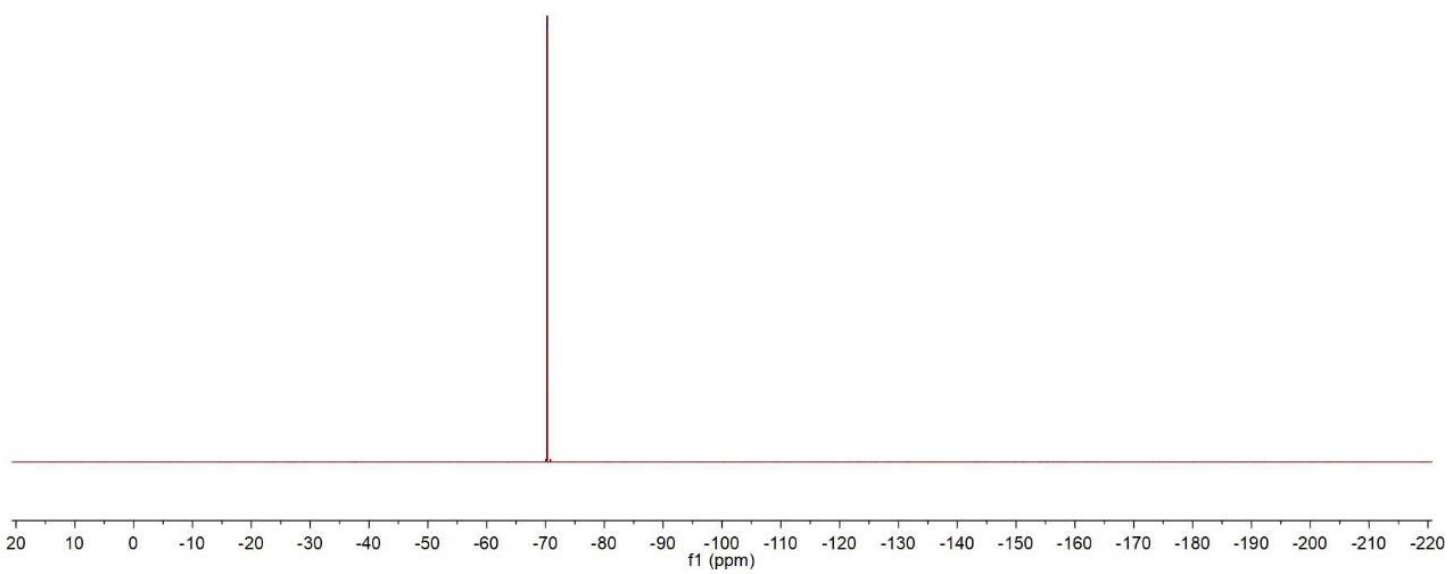


${ }^{1} \mathrm{H}$ NMR (400 MHz, $\mathrm{CDCl}_{3}$ ) spectrum for $3 \mathrm{i}$

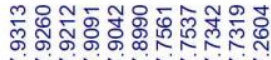

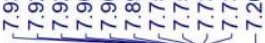<smiles>O=C(c1ccc(I)cc1)C(F)(F)F</smiles>

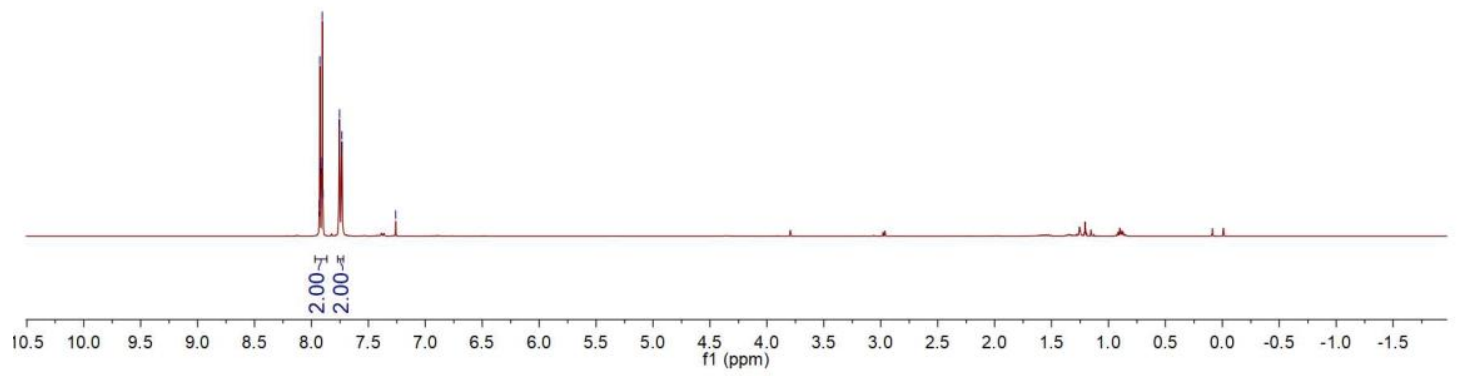

${ }^{13} \mathrm{C}$ NMR (100 MHz, $\mathrm{CDCl}_{3}$ ) spectrum for 3i

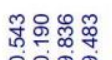

언욘?

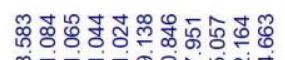

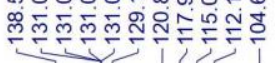<smiles>O=C(c1ccc(I)cc1)C(F)(F)F</smiles>

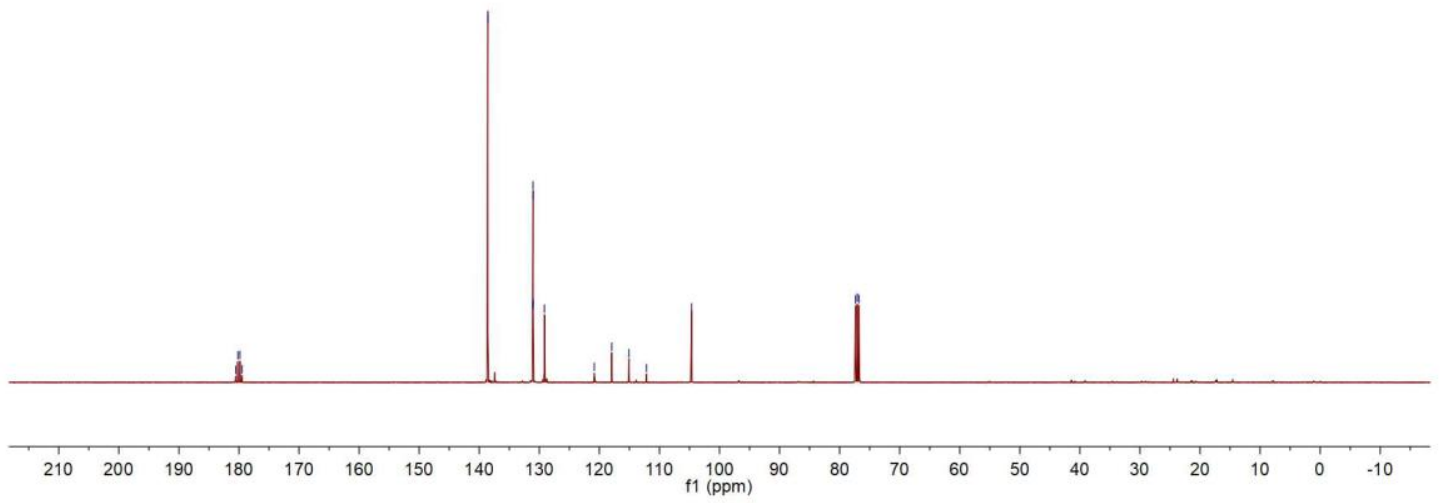


${ }^{19}$ F NMR (376 MHz, $\mathrm{CDCl}_{3}$ ) spectrum for $3 \mathrm{i}$

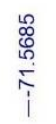<smiles>O=C(c1ccc(I)cc1)C(F)(F)F</smiles>

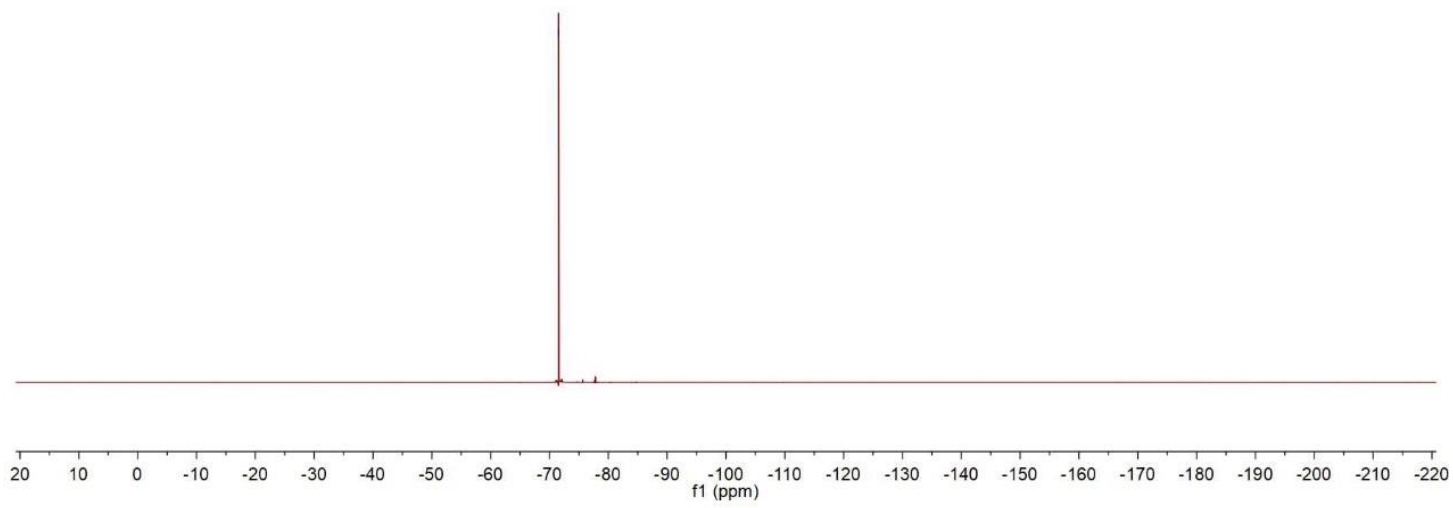

${ }^{1} \mathrm{H}$ NMR (400 MHz, $\mathrm{CDCl}_{3}$ ) spectrum for $3 \mathbf{j}$

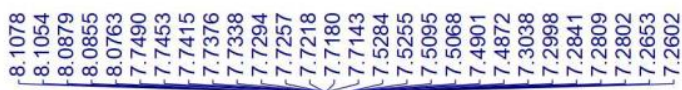<smiles>O=C(c1ccccc1I)C(F)(F)F</smiles>

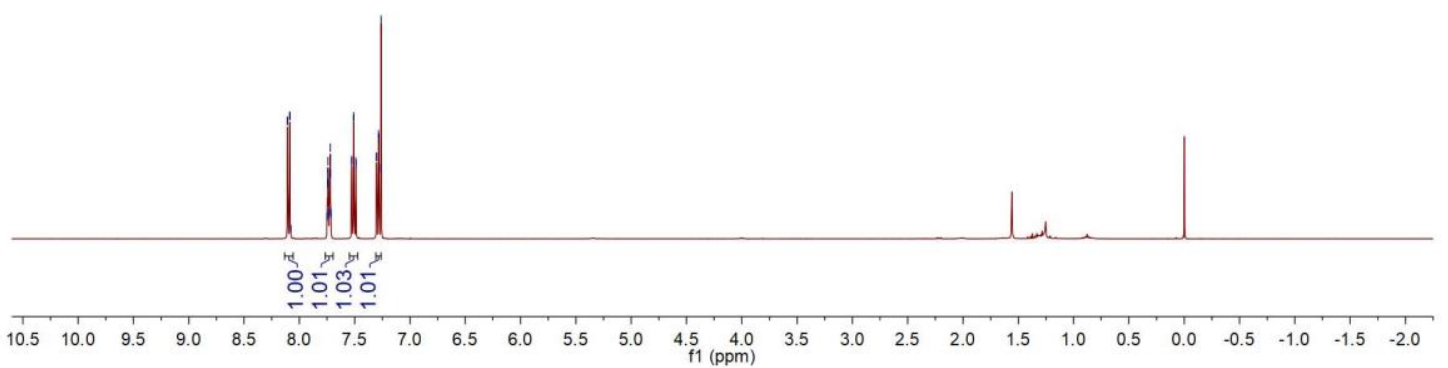


${ }^{13} \mathrm{C}$ NMR (100 MHz, $\left.\mathrm{CDCl}_{3}\right)$ spectrum for $3 \mathrm{j}$

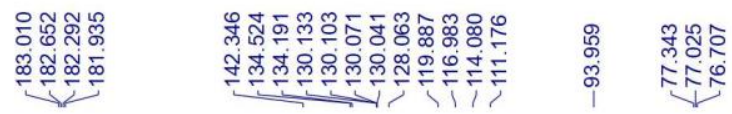<smiles>O=C(c1ccccc1I)C(F)(F)F</smiles>

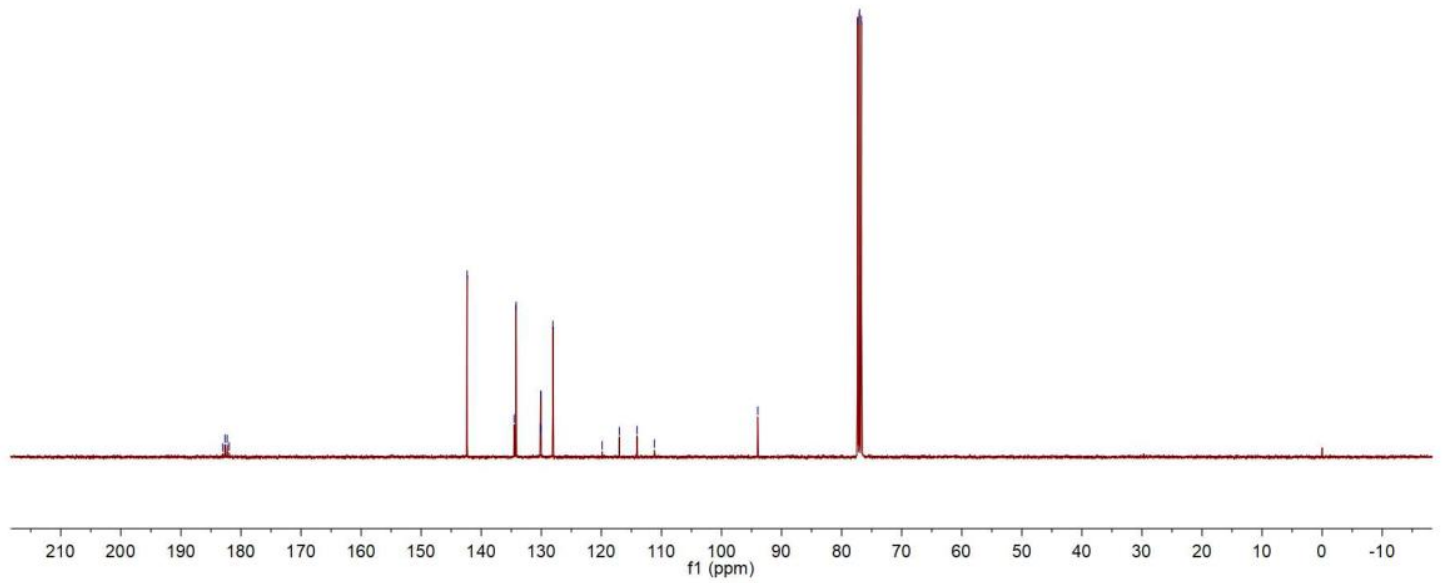

${ }^{19}$ F NMR (376 MHz, $\mathrm{CDCl}_{3}$ ) spectrum for $3 \mathrm{j}$<smiles>O=C(c1ccccc1I)C(F)(F)F</smiles>

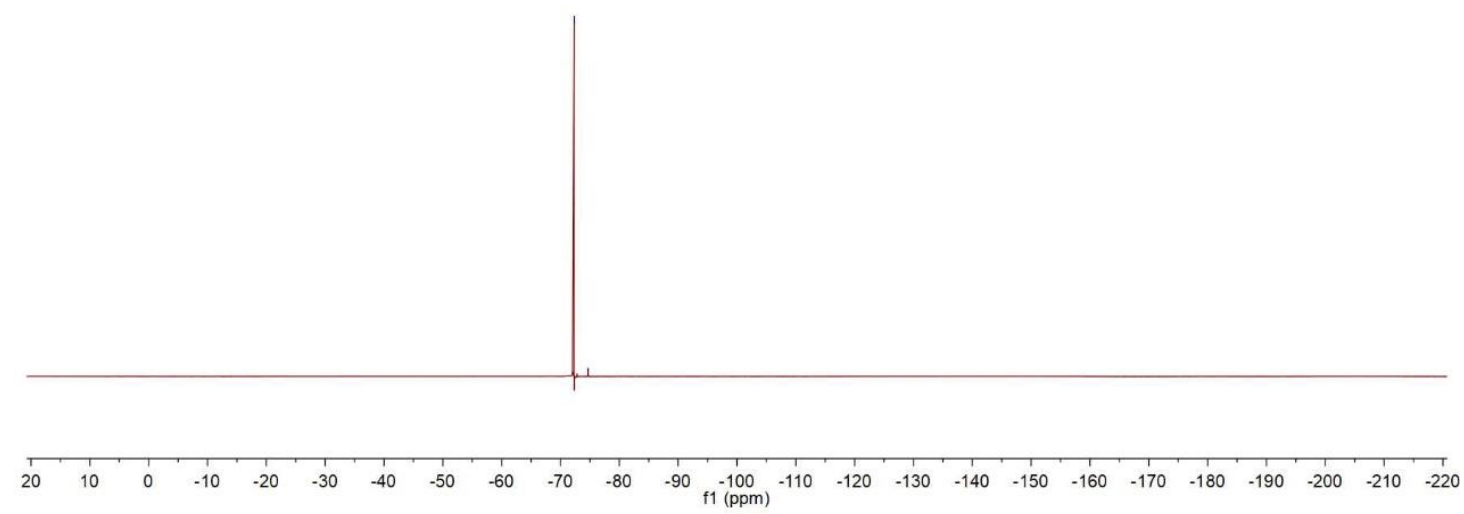


${ }^{1} \mathrm{H}$ NMR (400 MHz, $\mathrm{CDCl}_{3}$ ) spectrum for $3 \mathrm{k}$

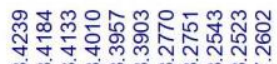

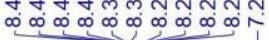<smiles>O=C(c1ccc([N+](=O)[O-])cc1)C(F)(F)F</smiles>

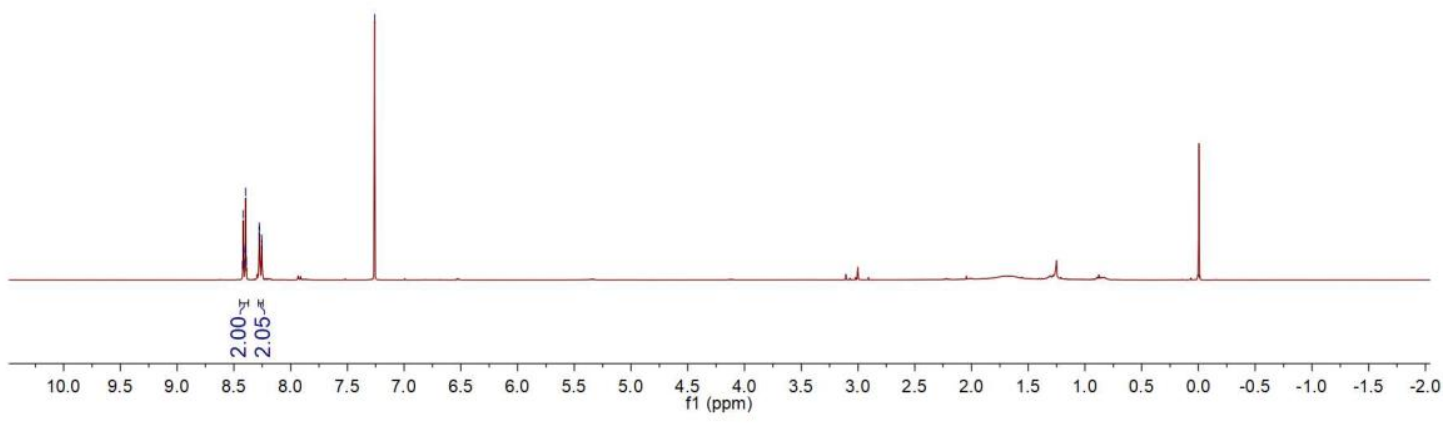

${ }^{13} \mathrm{C}$ NMR (100 MHz, $\left.\mathrm{CDCl}_{3}\right)$ spectrum for $3 \mathrm{k}$

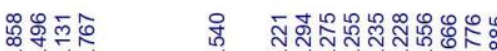

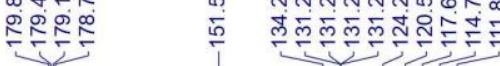

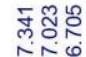

제유<smiles>O=C(c1ccc([N+](=O)[O-])cc1)C(F)(F)F</smiles>

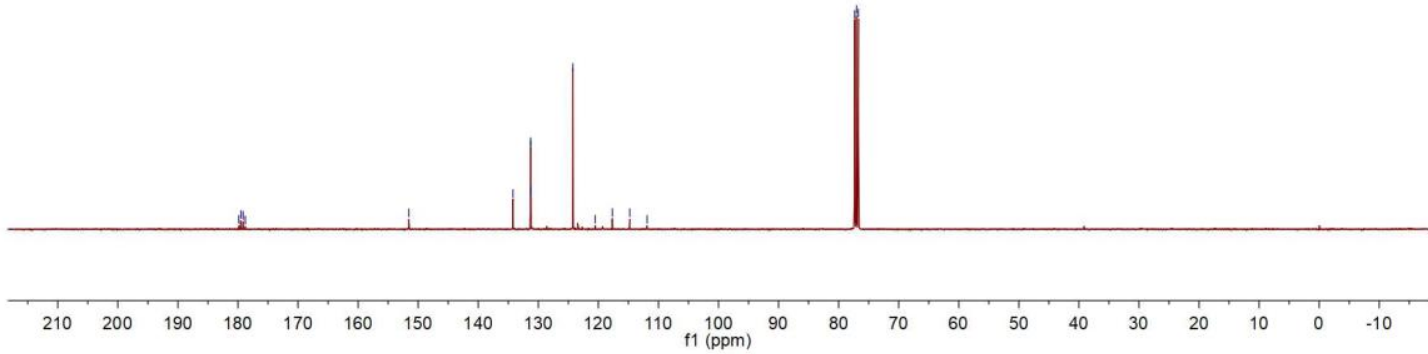


${ }^{19}$ F NMR (376 MHz, CDCl 3 ) spectrum for 3k

$$
\stackrel{\substack{\infty \\ \infty}}{\substack{i \\ \hdashline}}
$$<smiles>O=C(c1ccc([N+](=O)[O-])cc1)C(F)(F)F</smiles>

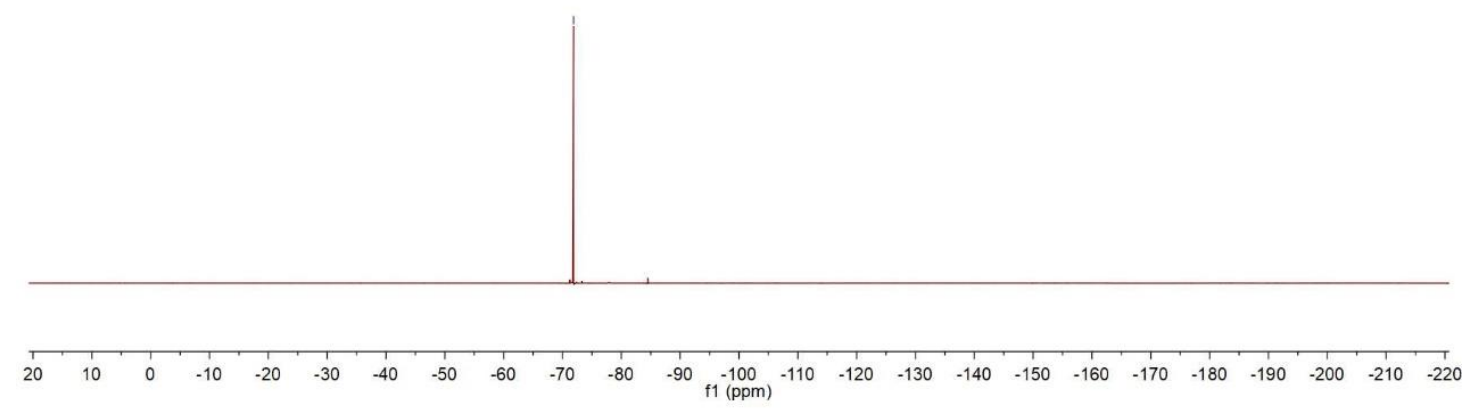

${ }^{1} \mathrm{H}$ NMR (400 MHz, $\mathrm{CDCl}_{3}$ ) spectrum for 31

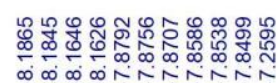

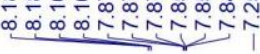<smiles>N#Cc1ccc(C(=O)C(F)(F)F)cc1</smiles>

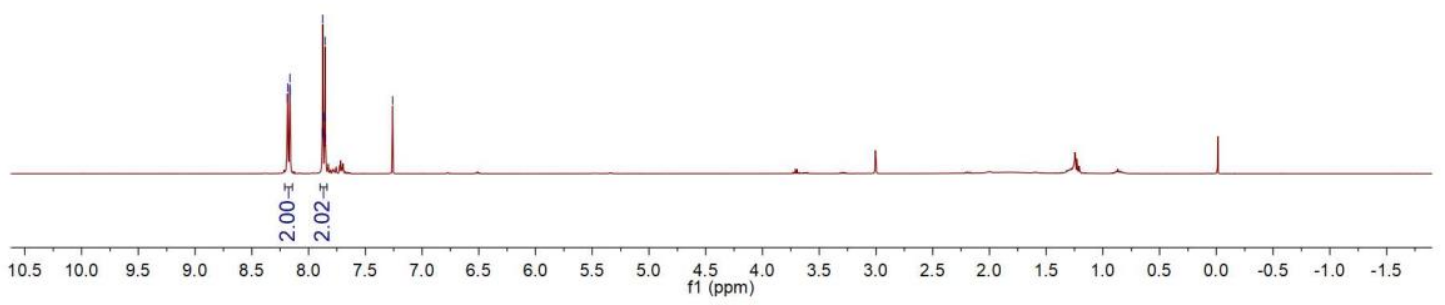


${ }^{13} \mathrm{C}$ NMR (100 MHz, $\mathrm{CDCl}_{3}$ ) spectrum for 31

\begin{tabular}{|c|c|}
\hline 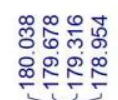 & 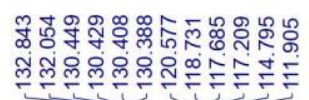 \\
\hline
\end{tabular}<smiles>N#Cc1ccc(C(=O)C(F)(F)F)cc1</smiles>

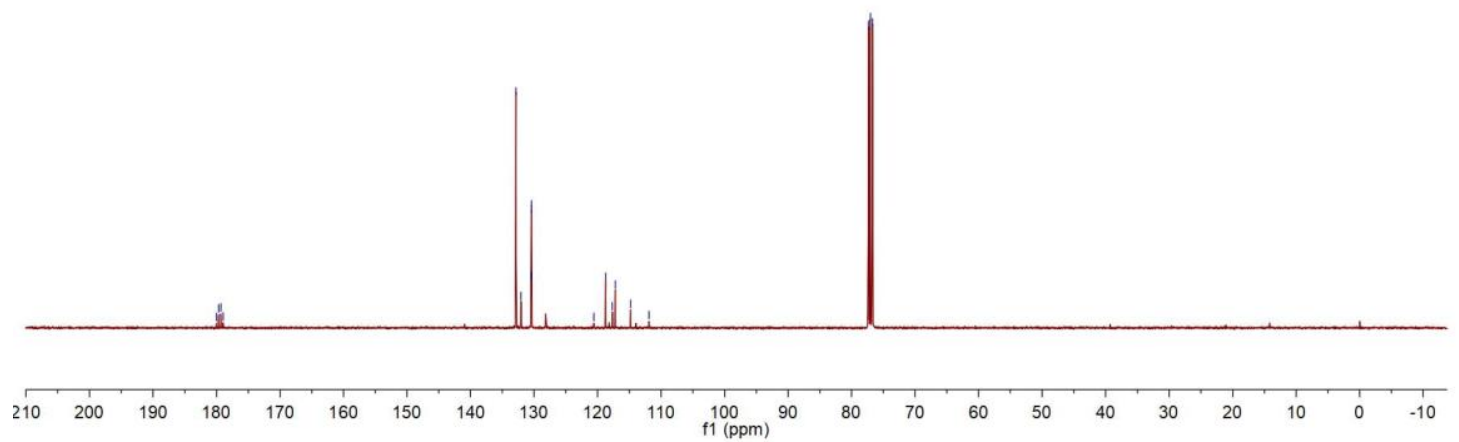

${ }^{19}$ F NMR (376 MHz, $\left.\mathrm{CDCl}_{3}\right)$ spectrum for 31<smiles>N#Cc1ccc(C(=O)C(F)(F)F)cc1</smiles>

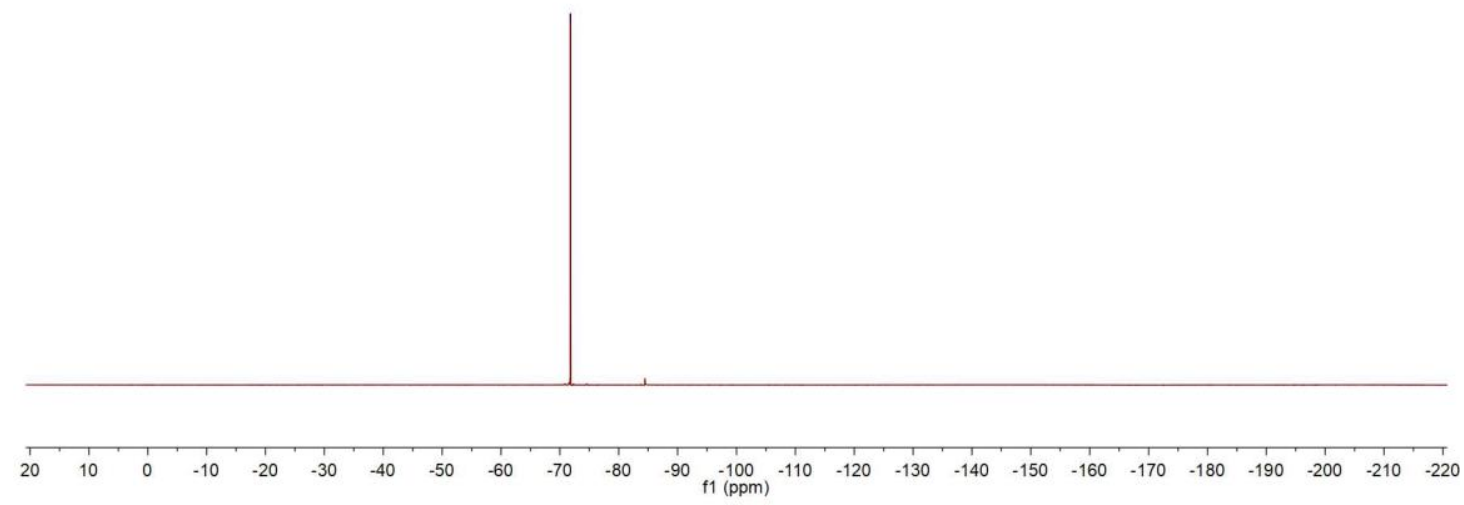


${ }^{1} \mathrm{H}$ NMR (400 MHz, $\mathrm{CDCl}_{3}$ ) spectrum for $3 \mathrm{~m}$

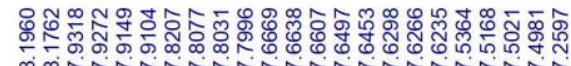
on<smiles>O=C(c1ccccc1)c1ccc(C(=O)C(F)(F)F)cc1</smiles>

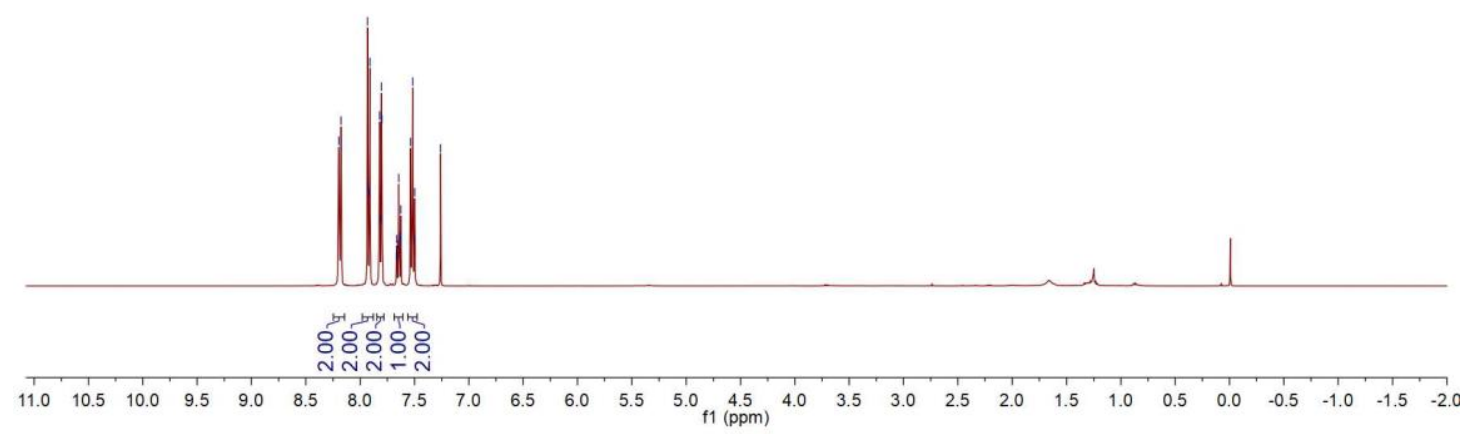

${ }^{13} \mathrm{C}$ NMR (100 $\left.\mathrm{MHz}, \mathrm{CDCl}_{3}\right)$ spectrum for $3 \mathrm{~m}$

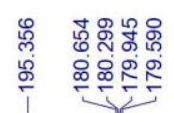

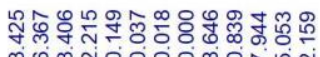
守过

휴용

N等<smiles>O=C(c1ccccc1)c1ccc(C(=O)C(F)(F)F)cc1</smiles>

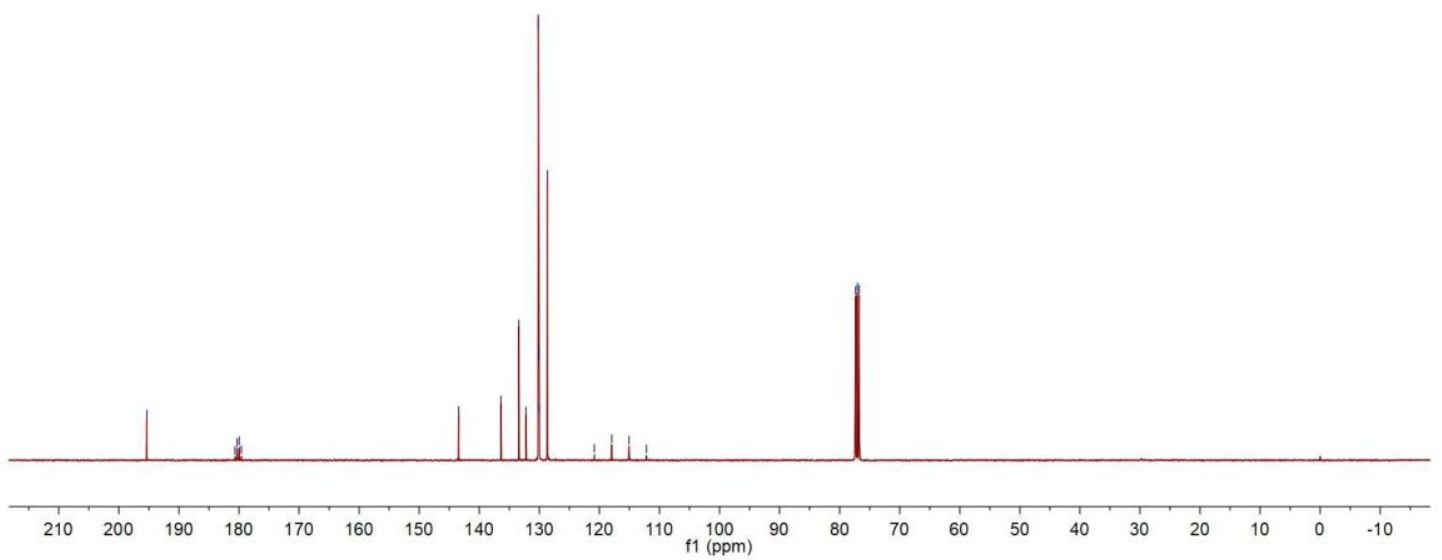


${ }^{19}$ F NMR (376 MHz, $\mathrm{CDCl}_{3}$ ) spectrum for $3 \mathrm{~m}$<smiles>O=C(c1ccccc1)c1ccc(C(=O)C(F)(F)F)cc1</smiles>

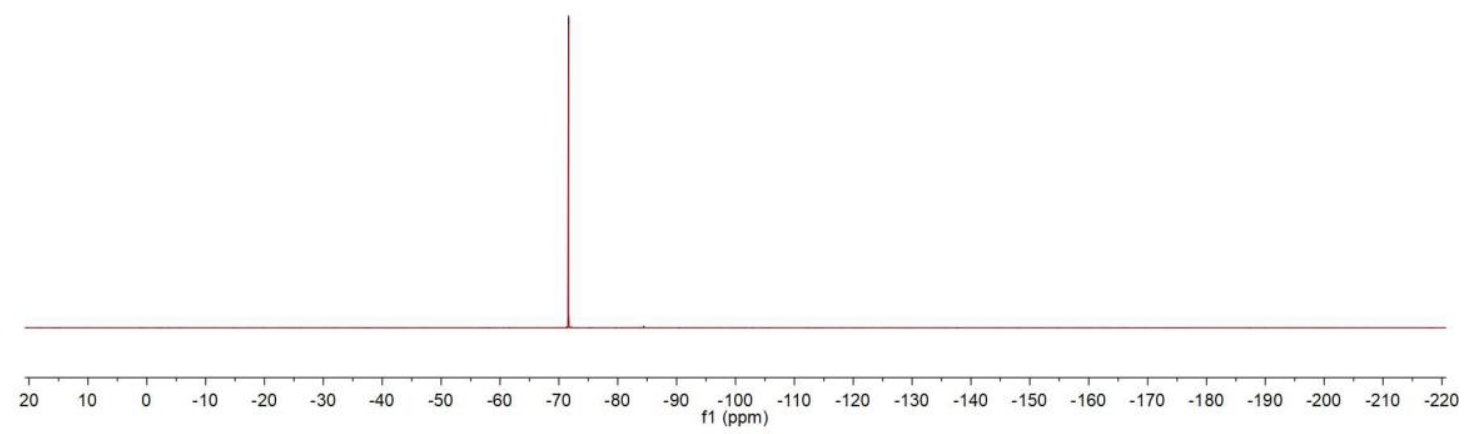

${ }^{1} \mathrm{H}$ NMR (400 MHz, $\mathrm{CDCl}_{3}$ ) spectrum for 3n

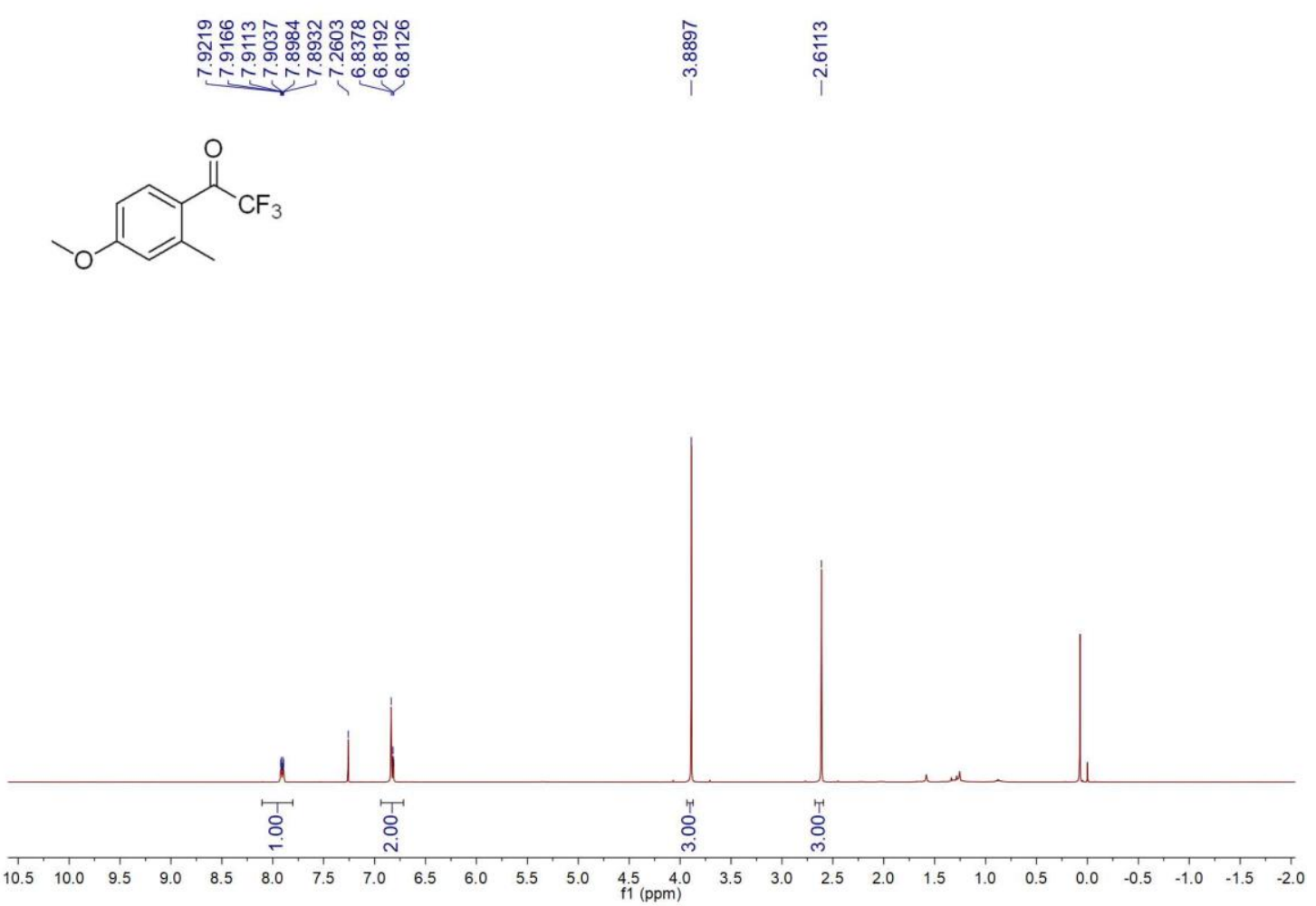


${ }^{13} \mathrm{C}$ NMR (100 MHz, CDCl 3$)$ spectrum for 3n

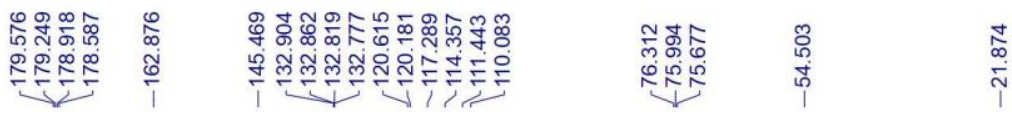<smiles>COc1ccc(C(=O)C(F)(F)F)c(C)c1</smiles>

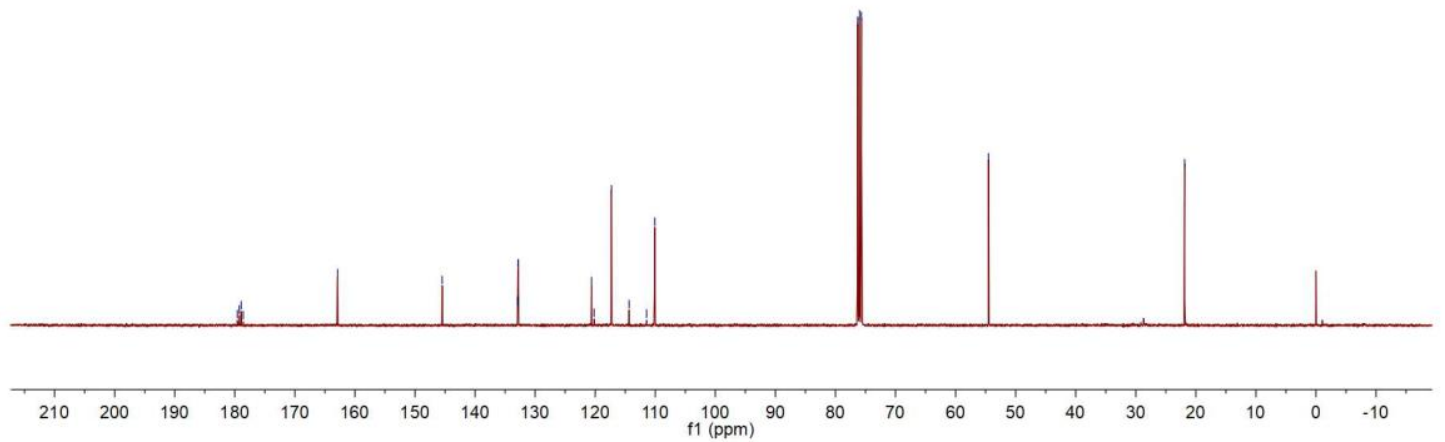

${ }^{19}$ F NMR (376 $\mathrm{MHz}, \mathrm{CDCl}_{3}$ ) spectrum for 3n<smiles>COc1ccc(C(=O)C(F)(F)F)c(C)c1</smiles>

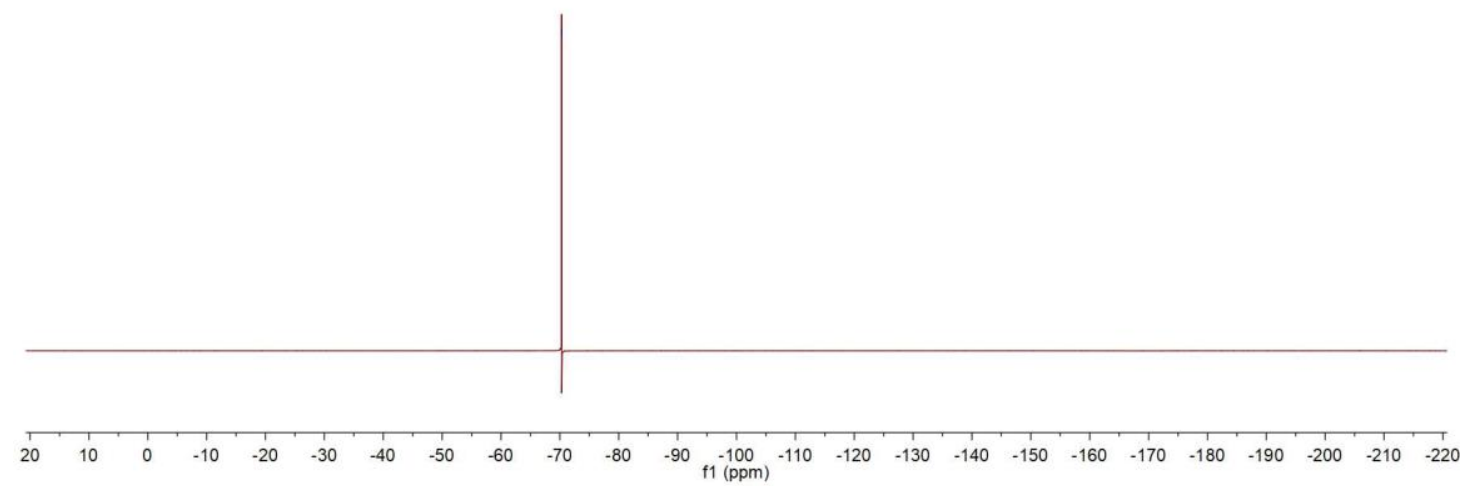


${ }^{1} \mathrm{H}$ NMR (400 MHz, $\mathrm{CDCl}_{3}$ ) spectrum for 30

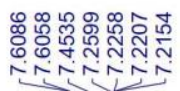<smiles>COc1cc(Cl)cc(C(=O)C(F)(F)F)c1</smiles>

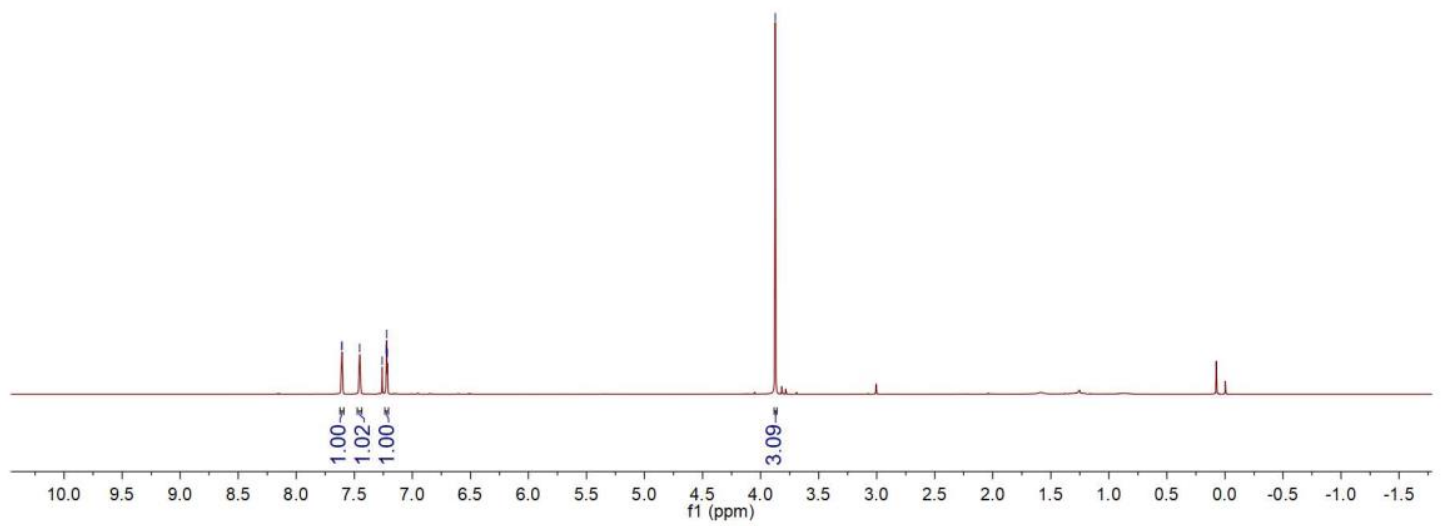

${ }^{13} \mathrm{C}$ NMR (100 MHz, $\mathrm{CDCl}_{3}$ ) spectrum for 30

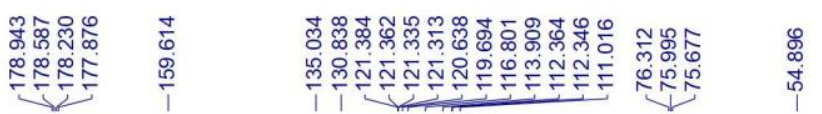<smiles>COc1cc(Cl)cc(C(=O)C(F)(F)F)c1</smiles>

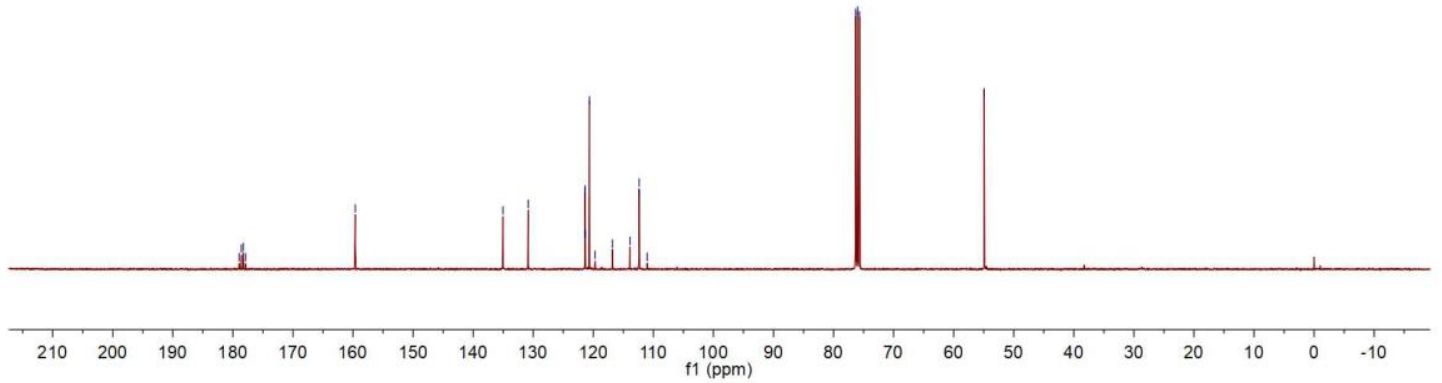


${ }^{19}$ F NMR (376 MHz, CDCl 3$)$ spectrum for 30

\&্ণ<smiles>COc1cc(Cl)cc(C(=O)C(F)(F)F)c1</smiles>

$\begin{array}{llllllllllllllllllllllllllllllll}20 & 10 & 0 & -10 & -20 & -30 & -40 & -50 & -60 & -70 & -80 & -90 & -10 & -110 & -120 & -130 & -140 & -150 & -160 & -170 & -180 & -190 & -200 & -210 & -220\end{array}$

${ }^{1} \mathrm{H}$ NMR (400 MHz, $\left.\mathrm{CDCl}_{3}\right)$ spectrum for 3p
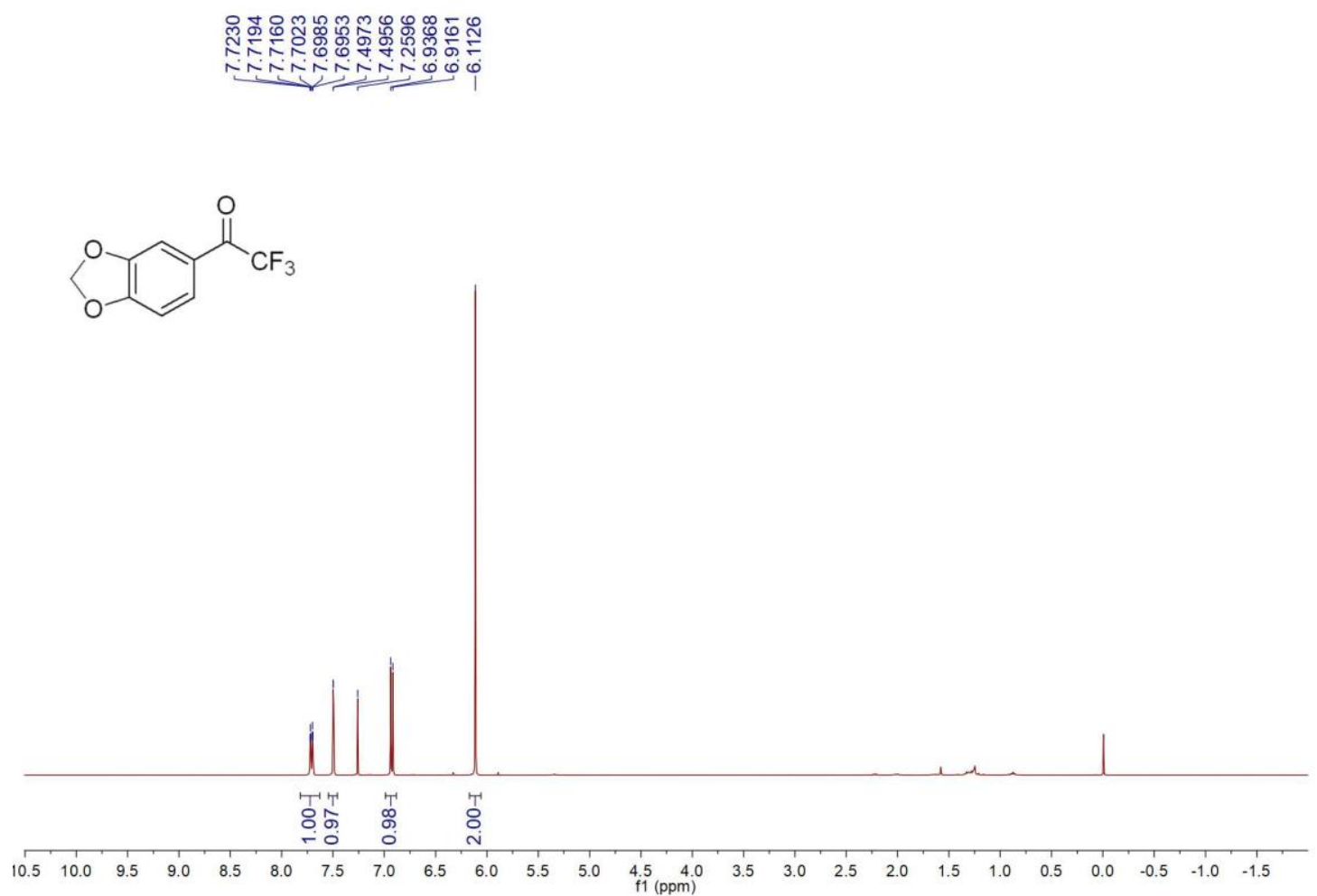
${ }^{13} \mathrm{C}$ NMR (100 MHz, $\left.\mathrm{CDCl}_{3}\right)$ spectrum for 3p

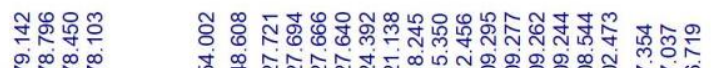

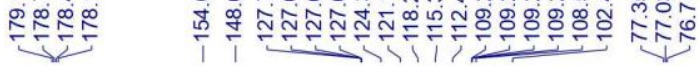
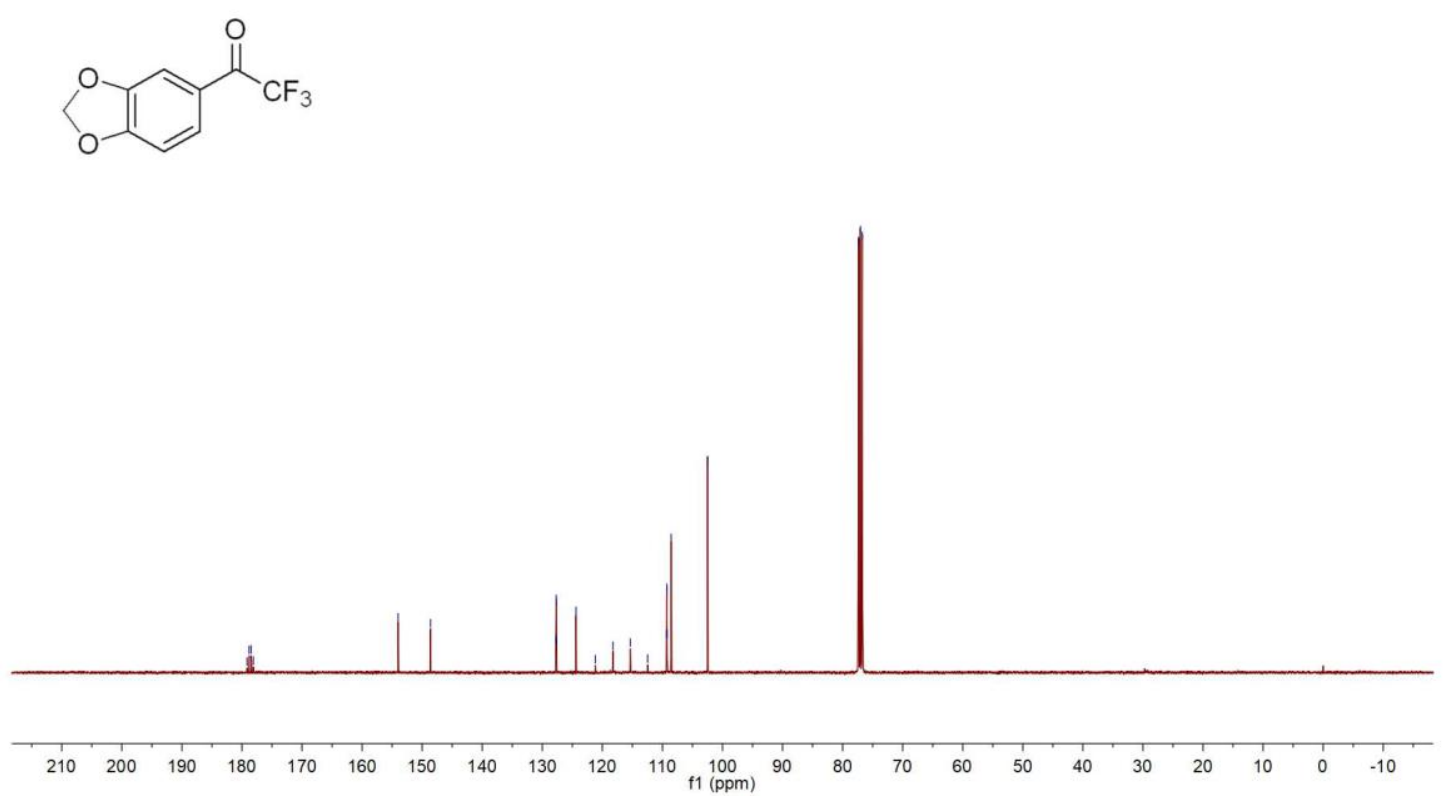

${ }^{19}$ F NMR (376 MHz, $\mathrm{CDCl}_{3}$ ) spectrum for $3 p$

$\hat{0}$
0
0
0
0<smiles>O=C([Te-])c1ccc2c(c1)OCO2</smiles>

$\begin{array}{lllllllllllllllllllllllllllllllll}20 & 10 & 0 & -10 & -20 & -30 & -40 & -50 & -60 & -70 & -80 & -90 & -100 & -110 & -120 & -130 & -140 & -150 & -160 & -170 & -180 & -190 & -200 & -210 & -220\end{array}$ 
${ }^{1} \mathrm{H}$ NMR (400 MHz, $\mathrm{CDCl}_{3}$ ) spectrum for $3 q$

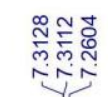<smiles>COc1cc(C(=O)C(F)(F)F)cc(OC)c1OC</smiles>

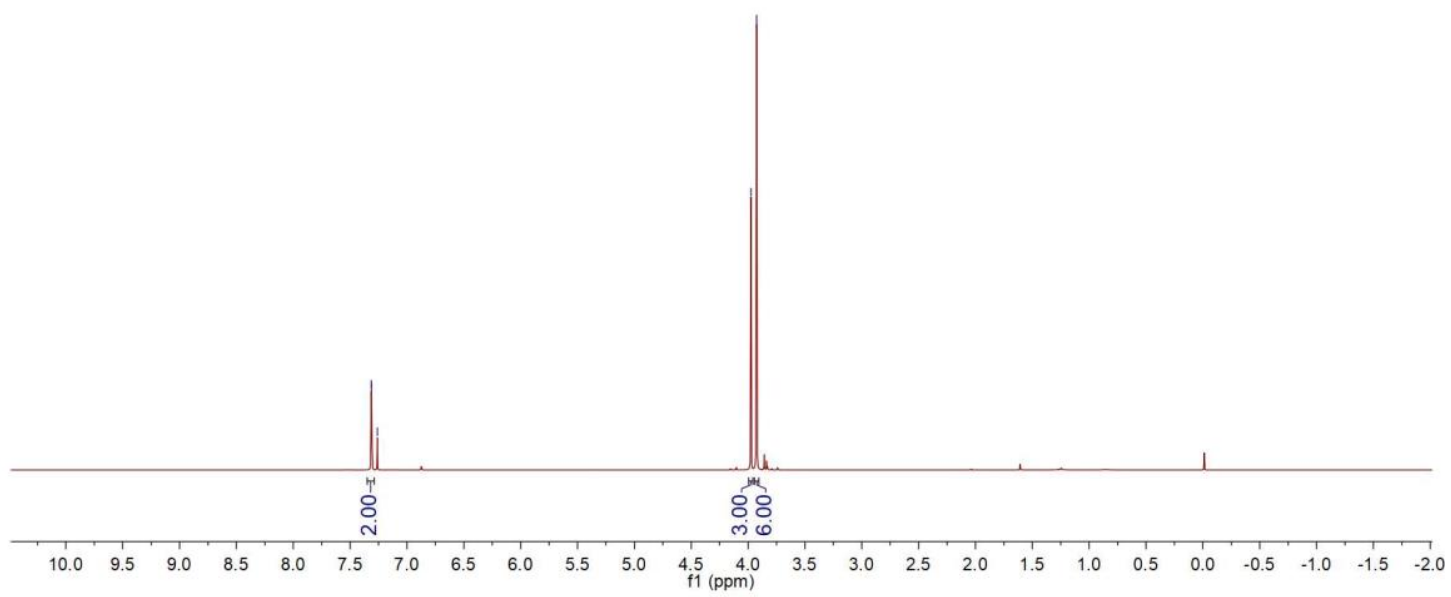

${ }^{13} \mathrm{C}$ NMR (100 MHz, $\left.\mathrm{CDCl}_{3}\right)$ spectrum for $3 q$

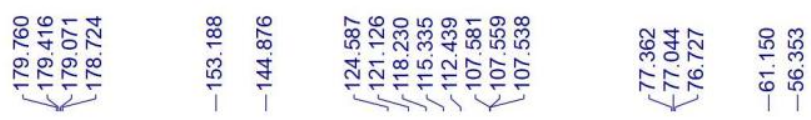<smiles>COc1cc(C(=O)C(F)(F)F)cc(OC)c1OC</smiles>

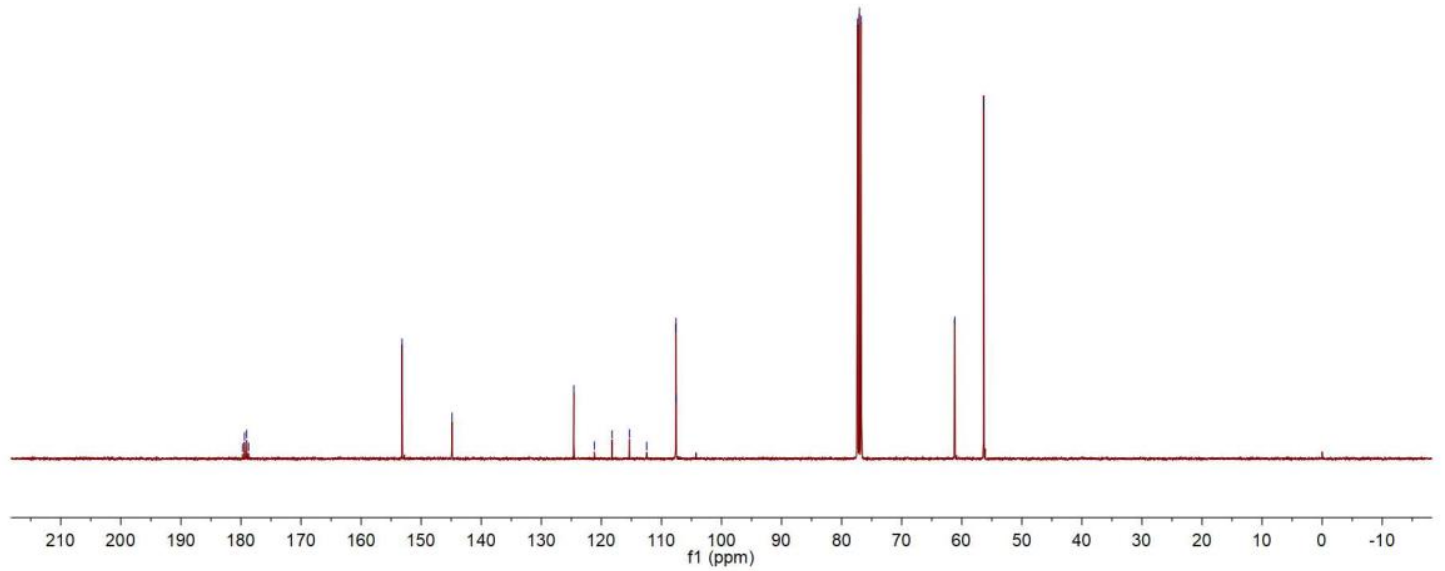


${ }^{19}$ F NMR (376 MHz, $\mathrm{CDCl}_{3}$ ) spectrum for 3q<smiles>COc1cc(C(=O)C(F)(F)F)cc(OC)c1OC</smiles>

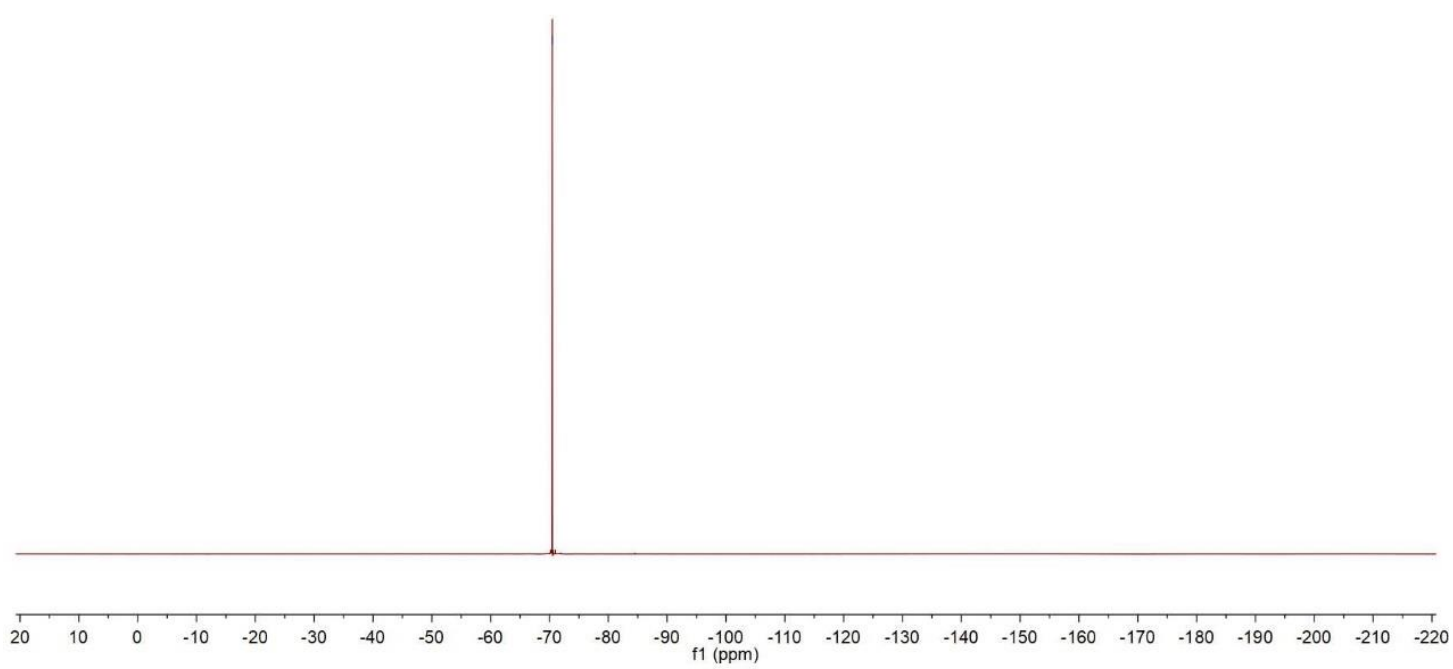

${ }^{1} \mathrm{H}$ NMR (400 MHz, $\mathrm{CDCl}_{3}$ ) spectrum for $3 \mathrm{r}$

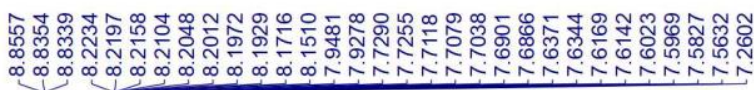<smiles>O=C(c1cccc2ccccc12)C(F)(F)C(F)(F)F</smiles>

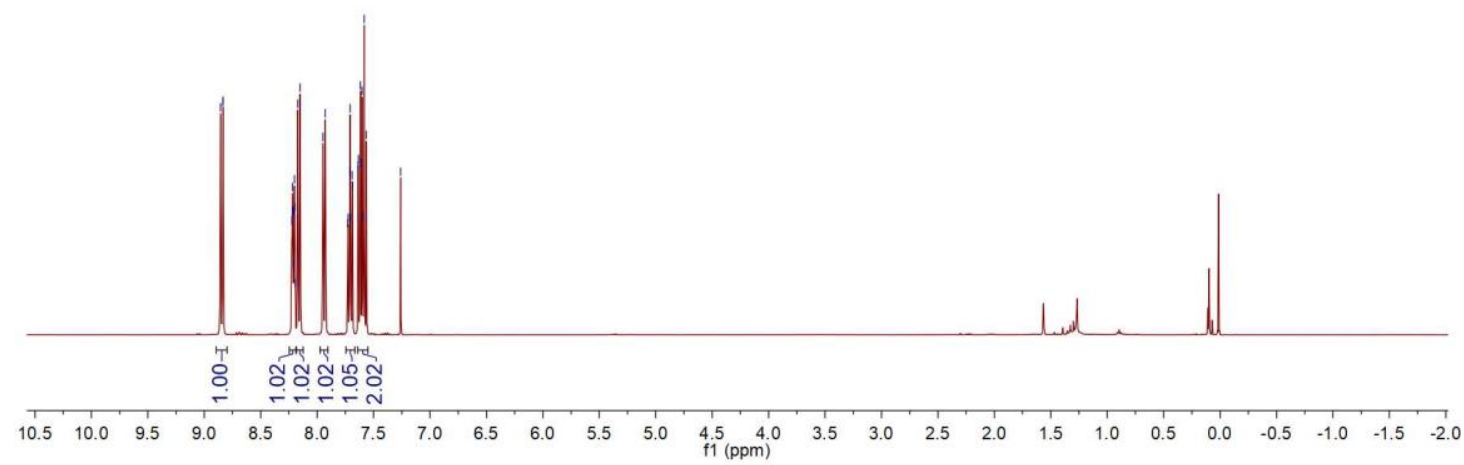


${ }^{13} \mathrm{C}$ NMR (100 MHz, $\mathrm{CDCl}_{3}$ ) spectrum for $3 \mathrm{r}$

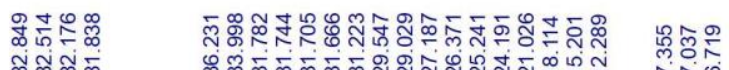

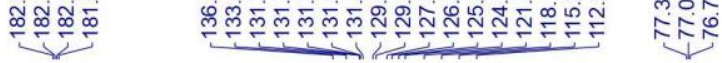<smiles>O=C(c1cccc2ccccc12)C(F)(F)F</smiles>

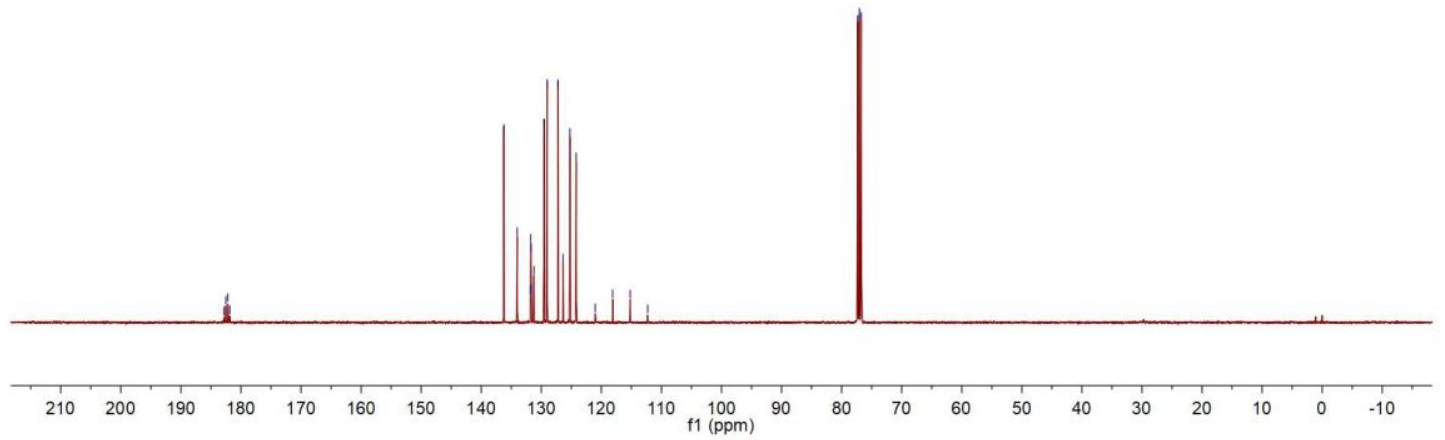

${ }^{19}$ F NMR (376 MHz, $\mathrm{CDCl}_{3}$ ) spectrum for $3 \mathrm{r}$<smiles></smiles><smiles>O=C(c1cccc2ccccc12)C(F)(F)F</smiles>

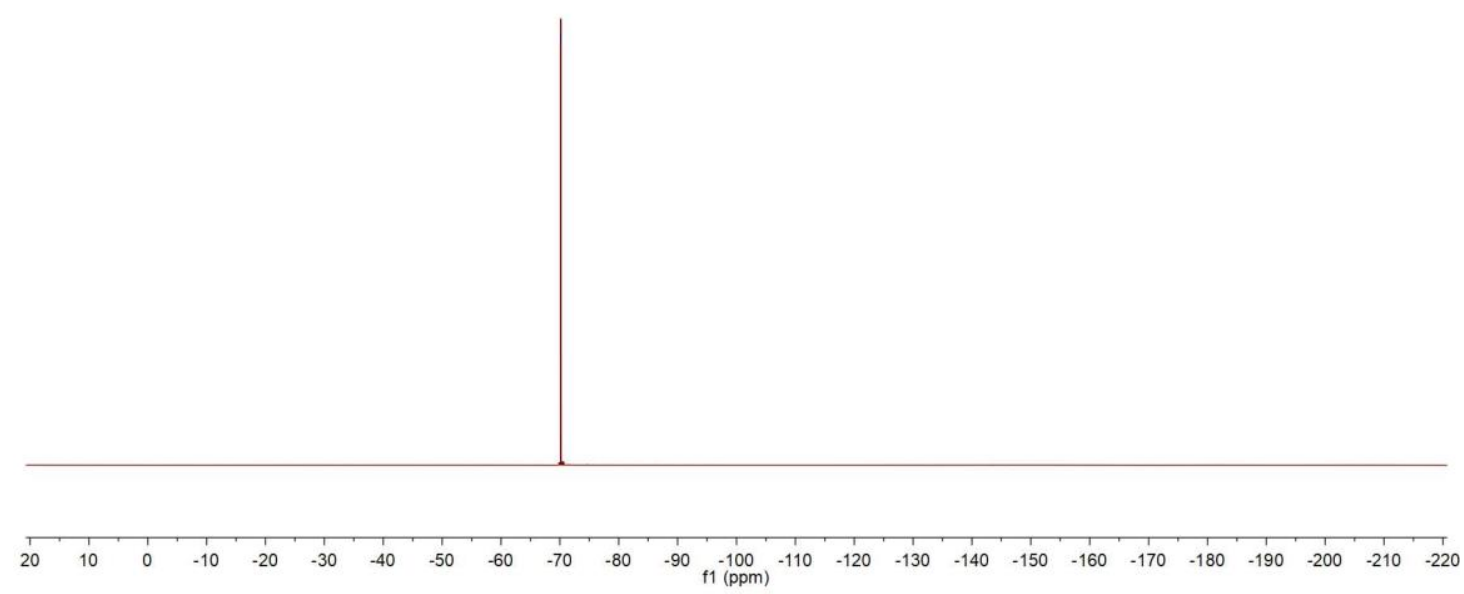


${ }^{1} \mathrm{H}$ NMR (400 MHz, $\mathrm{CDCl}_{3}$ ) spectrum for $3 \mathrm{~s}$

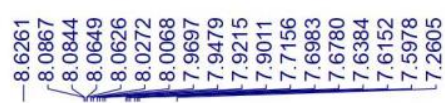<smiles>O=C(c1ccc2ccccc2c1)C(F)(F)F</smiles>

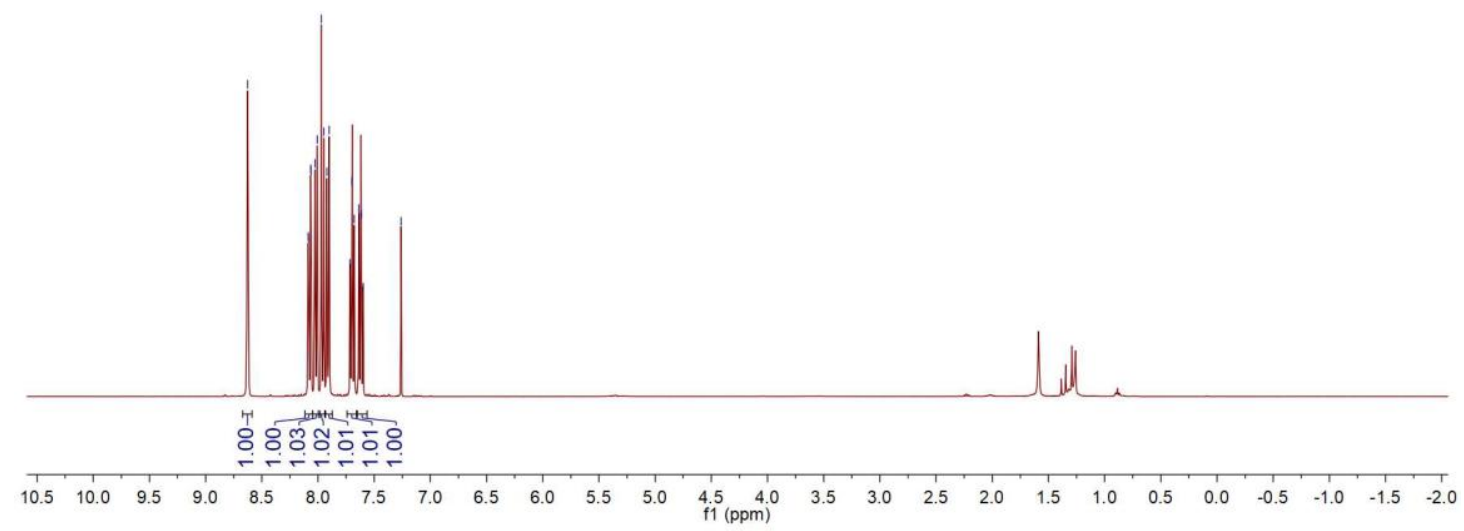

${ }^{13} \mathrm{C}$ NMR (100 MHz, $\left.\mathrm{CDCl}_{3}\right)$ spectrum for $3 \mathrm{~s}$

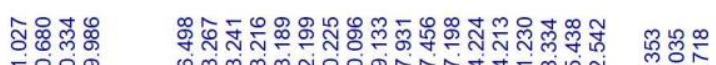

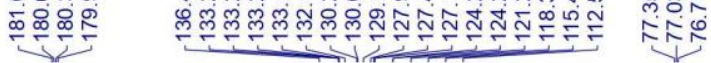<smiles>O=C(c1ccc2ccccc2c1)C(F)(F)F</smiles>

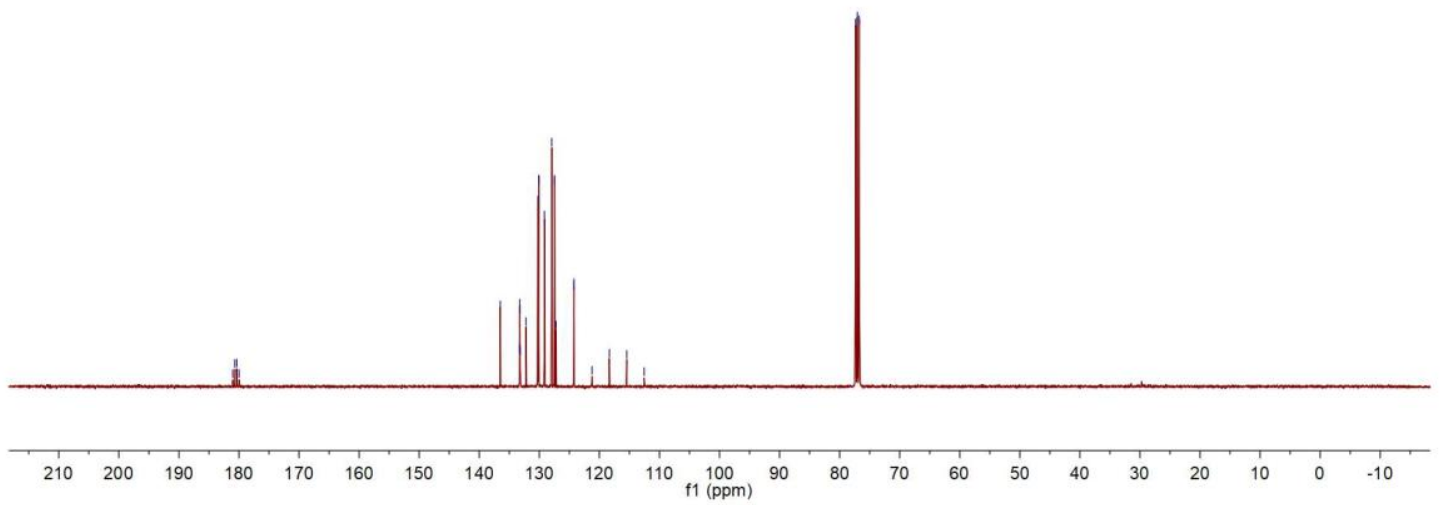


${ }^{19}$ F NMR (376 MHz, $\mathrm{CDCl}_{3}$ ) spectrum for $3 \mathrm{~s}$

离<smiles>O=C(c1ccc2ccccc2c1)C(F)(F)F</smiles>

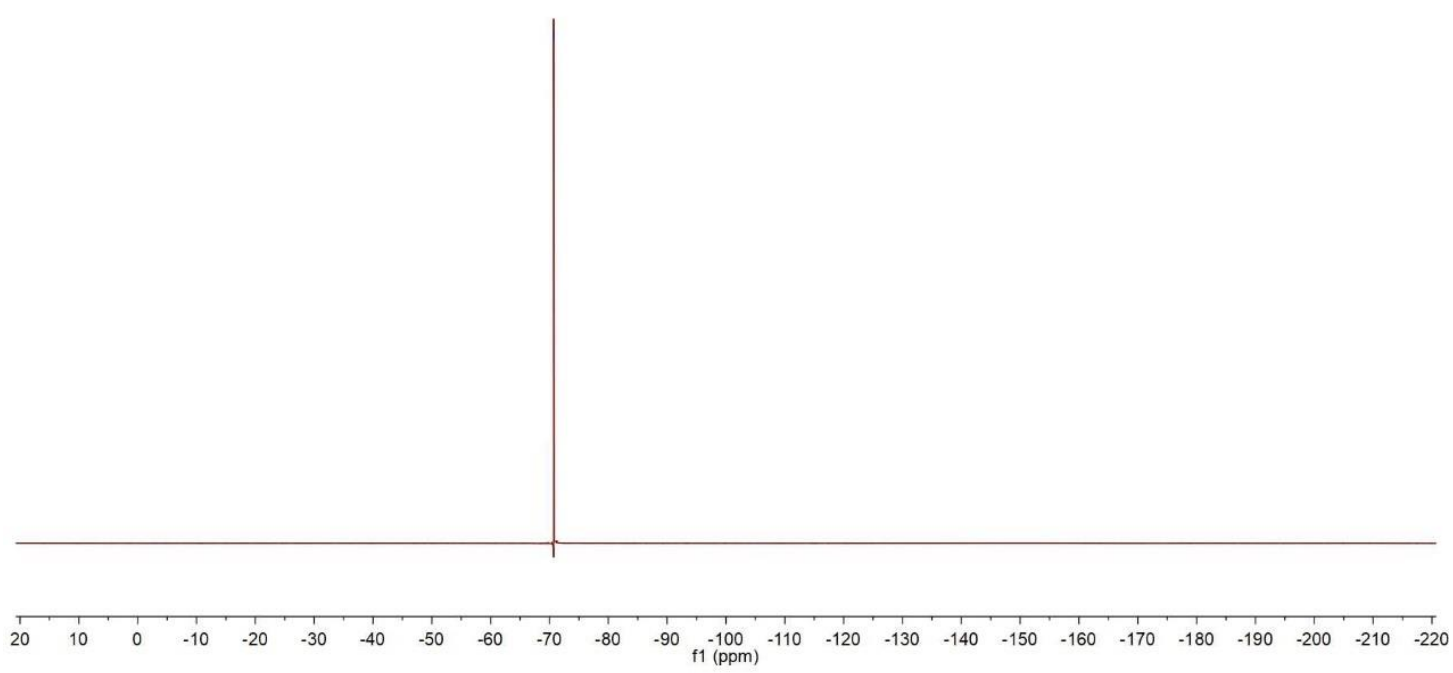

${ }^{1} \mathrm{H}$ NMR (400 MHz, $\mathrm{CDCl}_{3}$ ) spectrum for $3 \mathrm{t}$

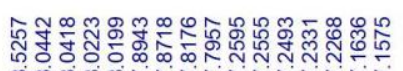

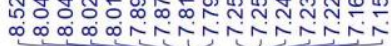<smiles>COc1ccc2cc(C(=O)C(F)(F)F)ccc2c1</smiles>

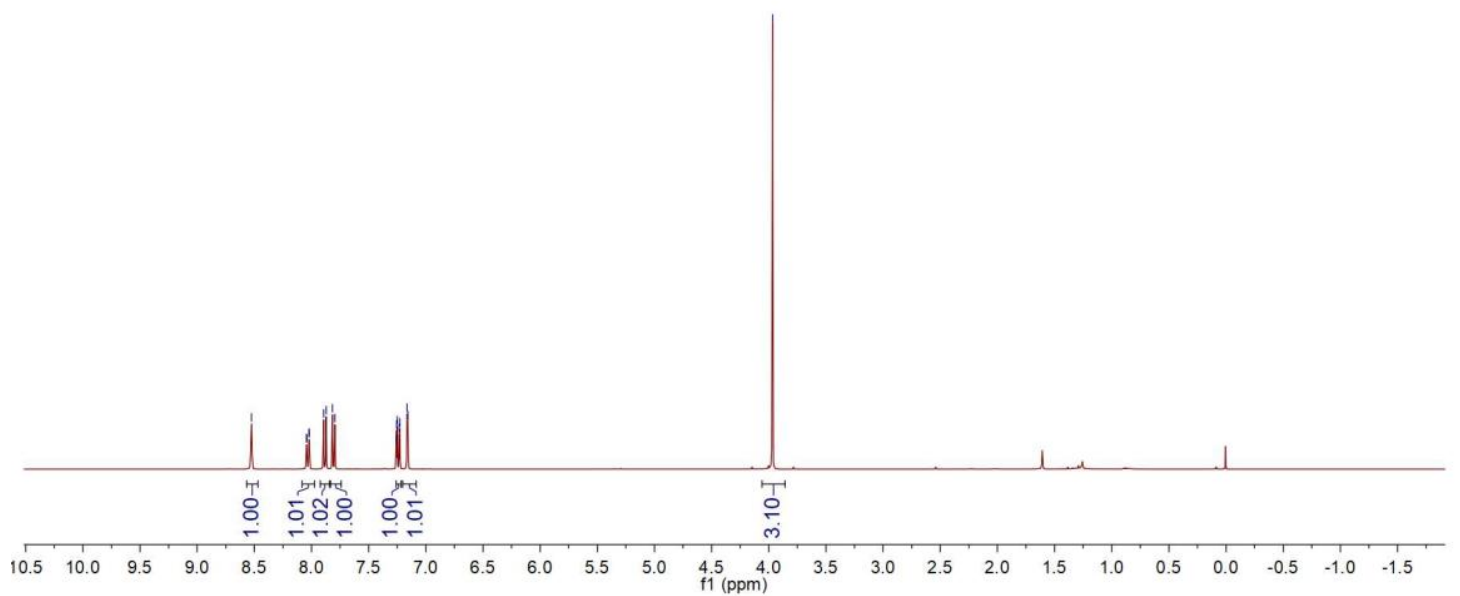


${ }^{13} \mathrm{C}$ NMR (100 MHz, $\mathrm{CDCl}_{3}$ ) spectrum for $3 \mathrm{t}$

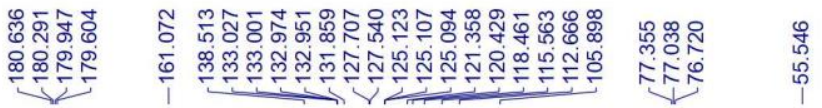<smiles>COc1ccc2cc(C(=O)C(F)(F)F)ccc2c1</smiles>

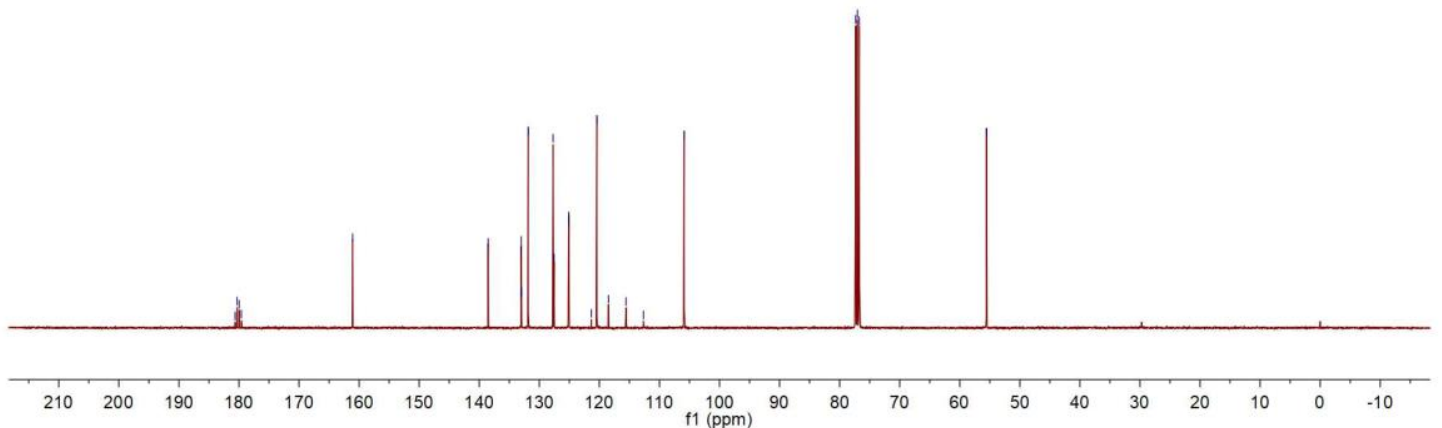

${ }^{19}$ F NMR (376 $\mathrm{MHz}, \mathrm{CDCl}_{3}$ ) spectrum for $3 \mathrm{t}$

总<smiles>COc1ccc2cc(C(=O)C(F)(F)F)ccc2c1</smiles>

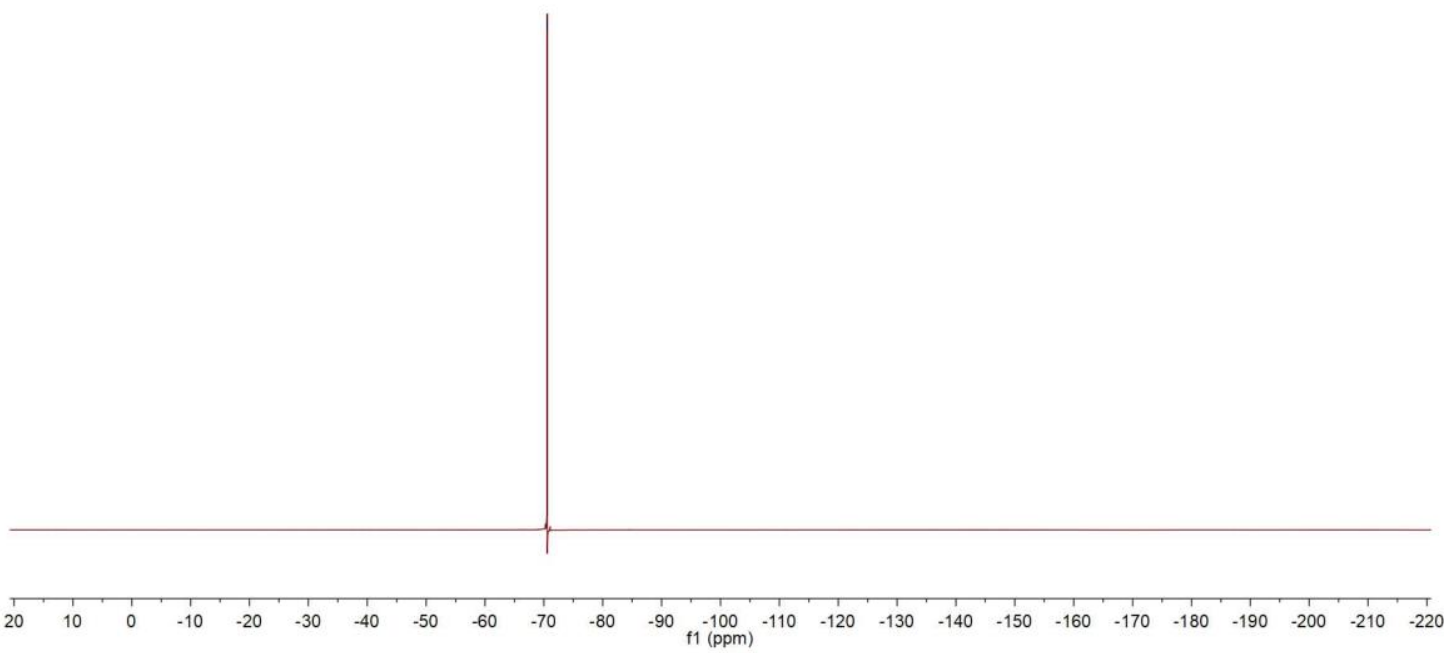


${ }^{1} \mathrm{H}$ NMR (400 MHz, $\mathrm{CDCl}_{3}$ ) spectrum for $3 \mathrm{u}$

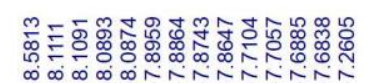

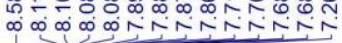<smiles>O=C(c1ccc2cc(Br)ccc2c1)C(F)(F)F</smiles>

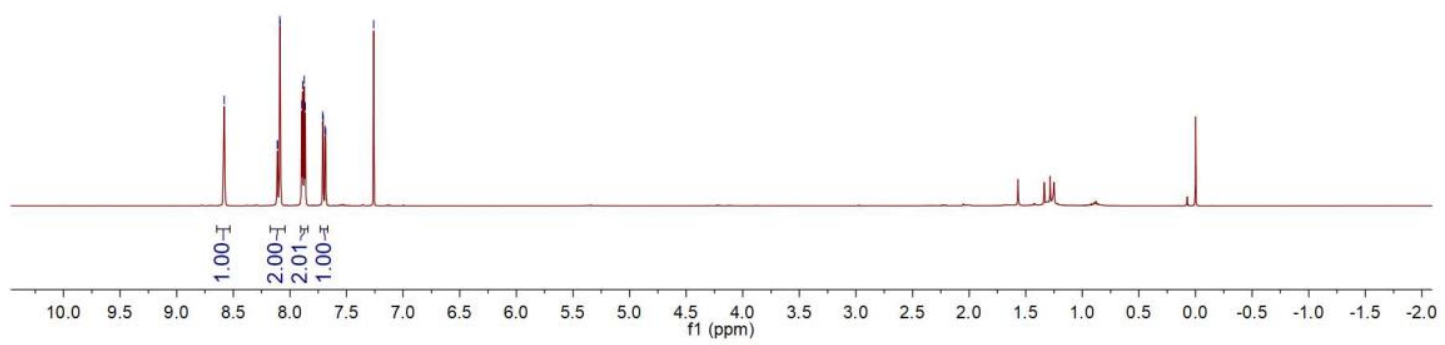

${ }^{13} \mathrm{C}$ NMR (100 MHz, $\left.\mathrm{CDCl}_{3}\right)$ spectrum for $3 \mathrm{u}$

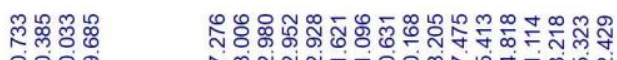

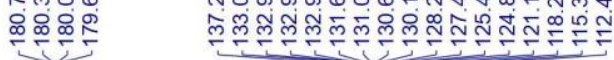<smiles>O=C(c1ccc2cc(Br)ccc2c1)C(F)(F)F</smiles>

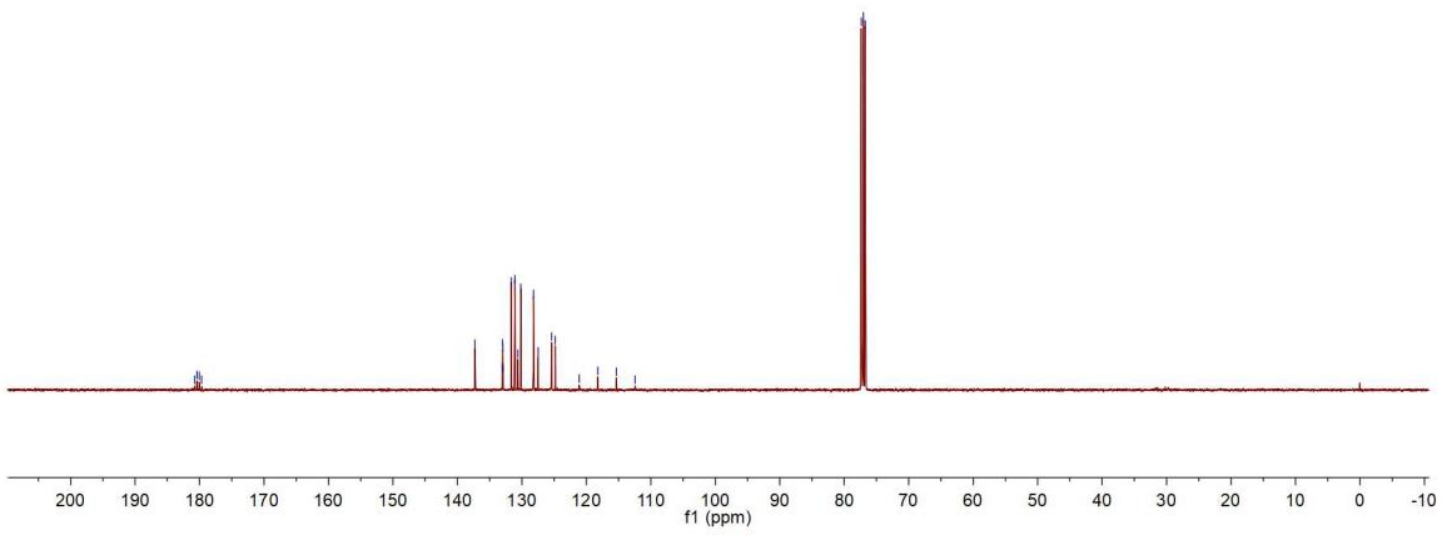


${ }^{19}$ F NMR (376 MHz, $\left.\mathrm{CDCl}_{3}\right)$ spectrum for $3 \mathrm{u}$<smiles>[C]1[C+]CC1</smiles><smiles>O=C(c1ccc2cc(Br)ccc2c1)C(F)(F)F</smiles>

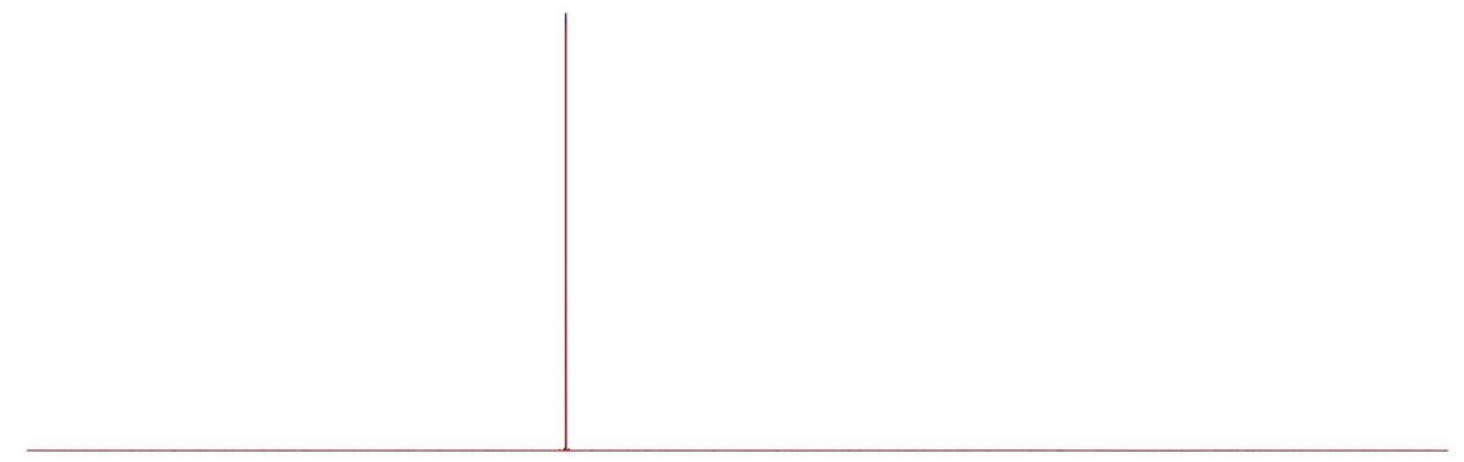

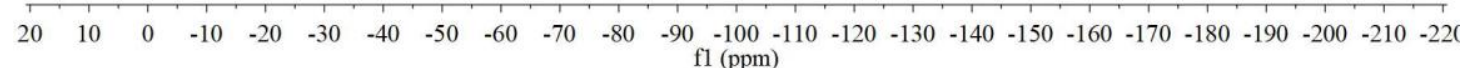

${ }^{1} \mathrm{H}$ NMR (400 MHz, $\mathrm{CDCl}_{3}$ ) spectrum for 3v

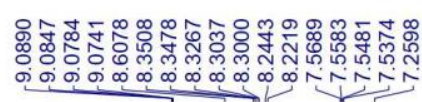<smiles>O=C(c1ccc2ncccc2c1)C(F)(F)F</smiles>

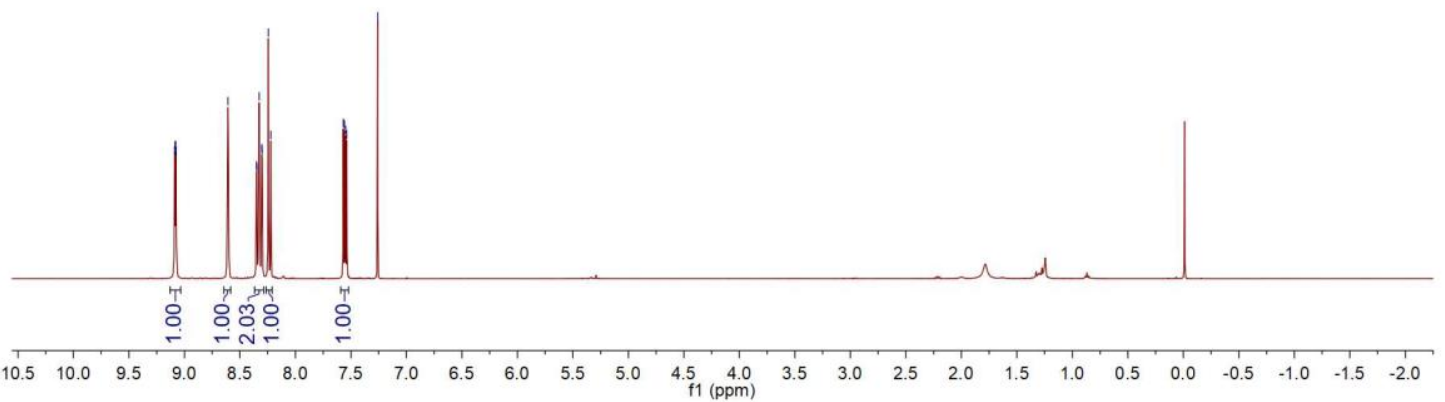


${ }^{13} \mathrm{C}$ NMR (100 MHz, $\mathrm{CDCl}_{3}$ ) spectrum for $3 \mathrm{v}$

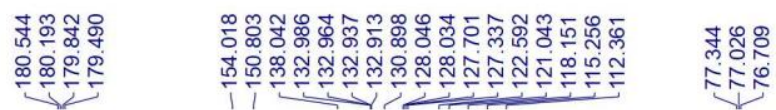
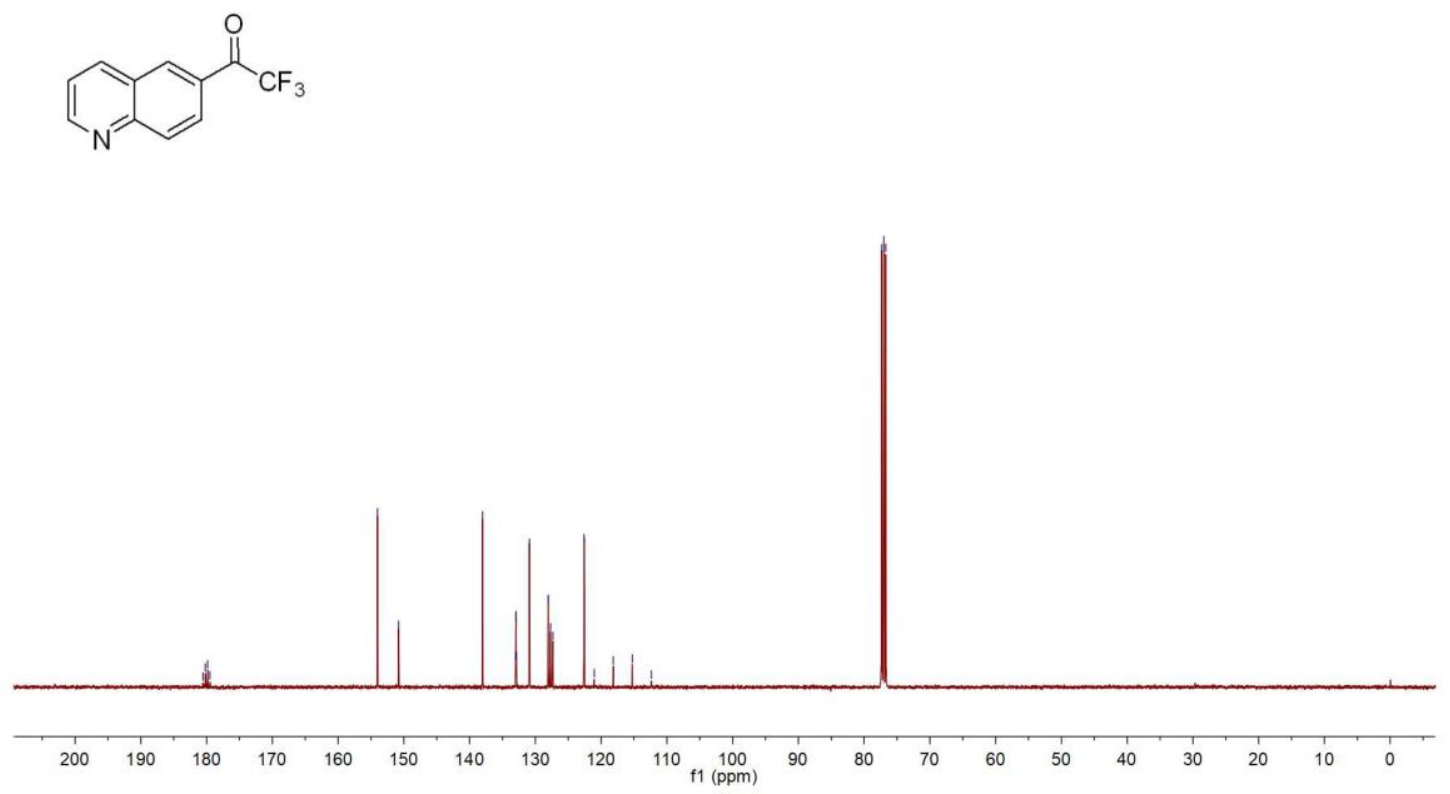

${ }^{19}$ F NMR (376 $\left.\mathrm{MHz}, \mathrm{CDCl}_{3}\right)$ spectrum for $3 \mathrm{v}$

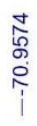<smiles>CC(F)(F)C(=O)c1ccc2ncccc2c1</smiles>

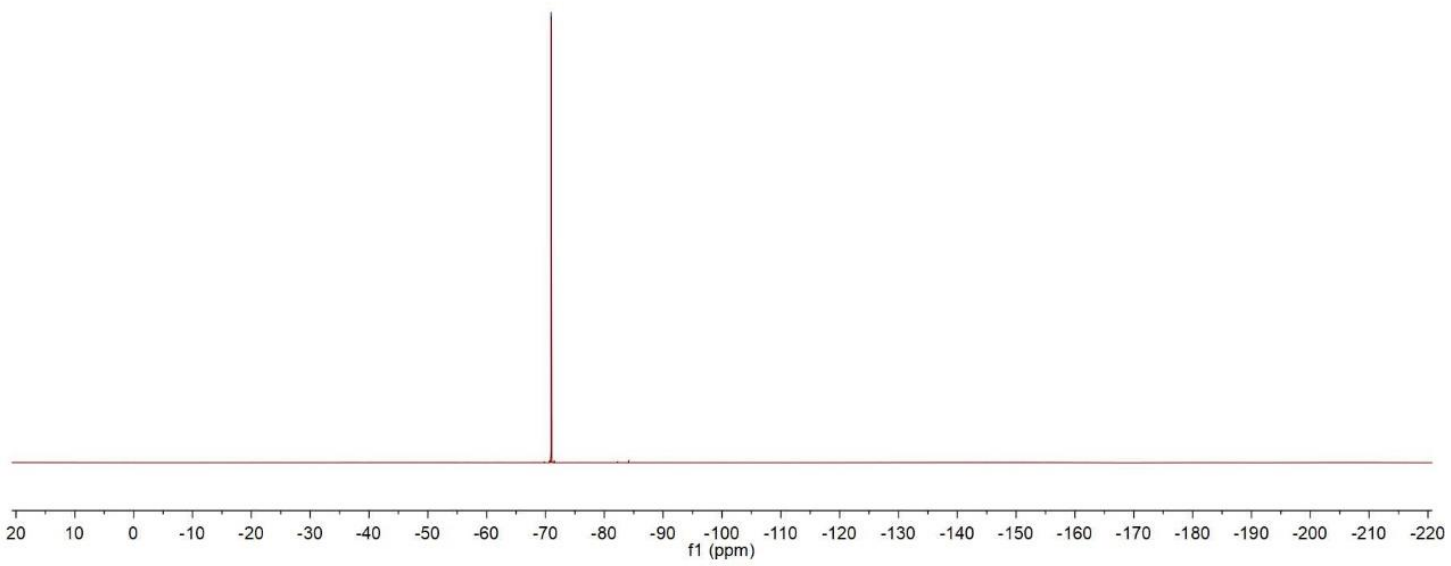


${ }^{1} \mathrm{H}$ NMR (400 MHz, $\mathrm{CDCl}_{3}$ ) spectrum for 3w

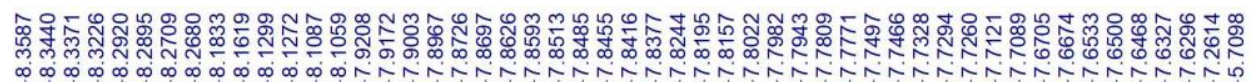
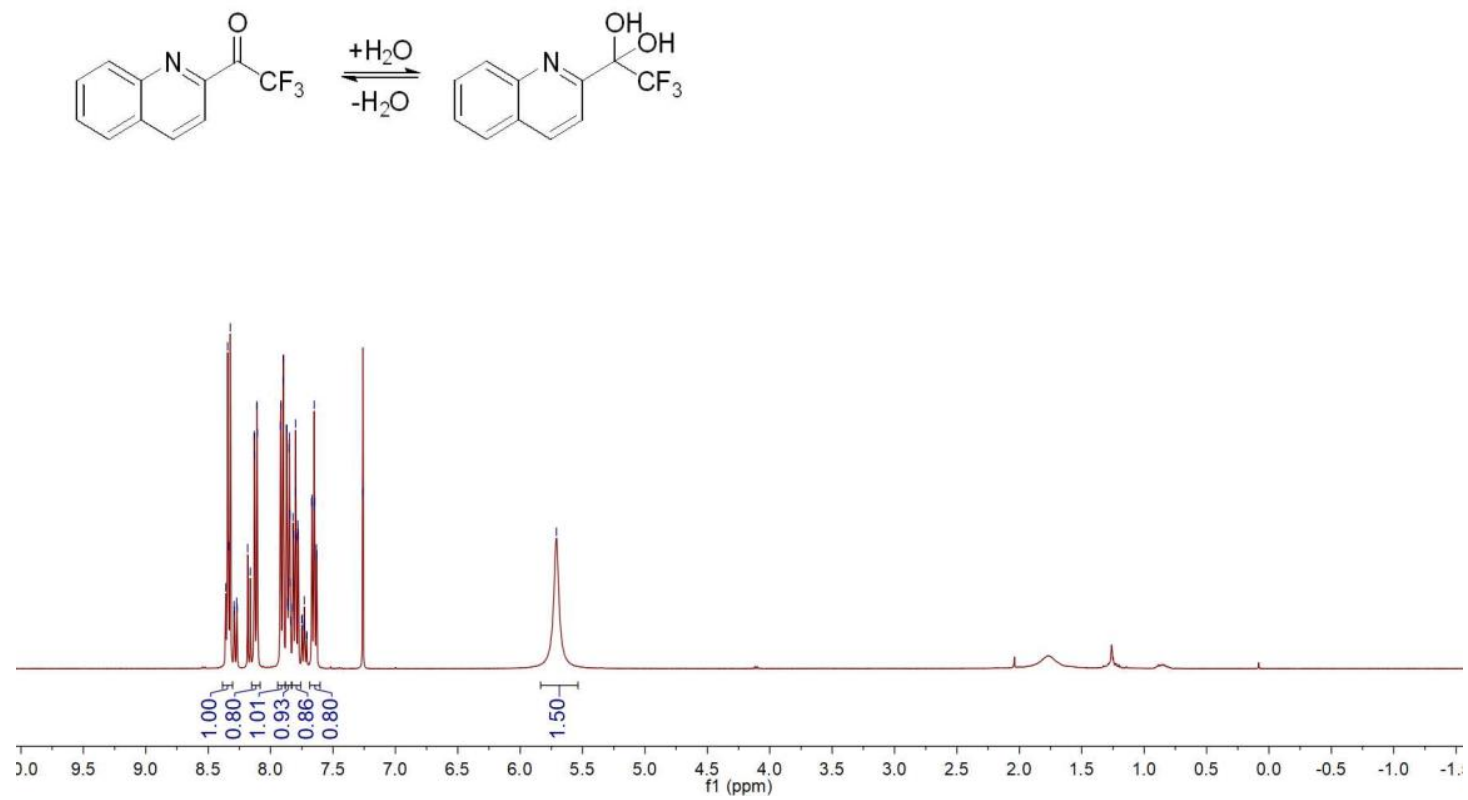

${ }^{13} \mathrm{C}$ NMR (100 MHz, $\left.\mathrm{CDCl}_{3}\right)$ spectrum for 3w

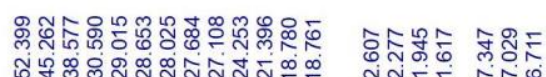

눈<smiles>O=C(c1ccc2ccccc2n1)C(F)(F)[C@H](O)C(O)O</smiles>

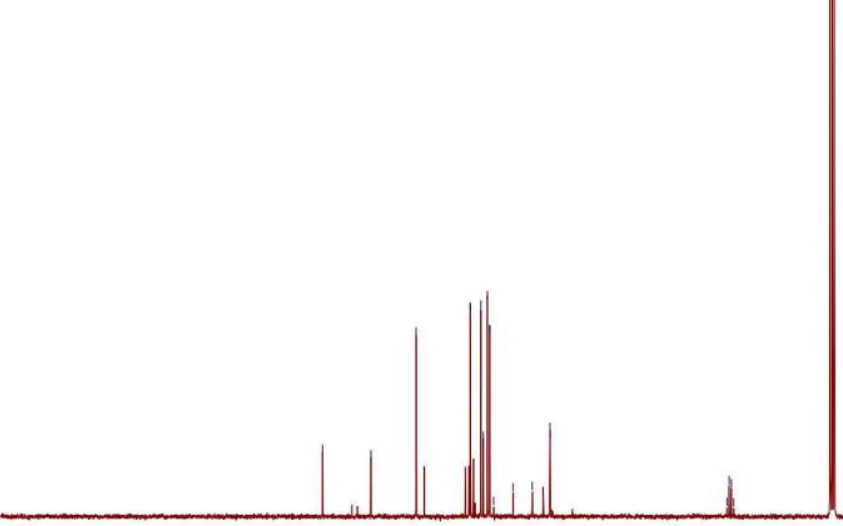

\begin{tabular}{|lllllllllllllllllllllll}
\hline 1 & 190 & 180 & 170 & 160 & 150 & 140 & 130 & 120 & 110 & $\underset{f 1}{1}(\mathrm{ppm})$ & 90 & 70 & 60 & 50 & 40 & 30 & 20 & 10 & 0 & -10
\end{tabular} 
${ }^{19}$ F NMR (376 MHz, $\mathrm{CDCl}_{3}$ ) spectrum for 3w

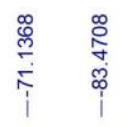

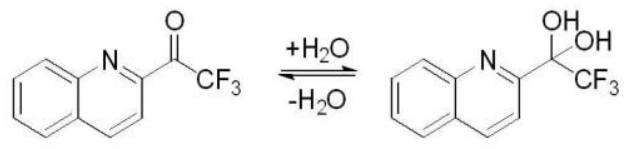

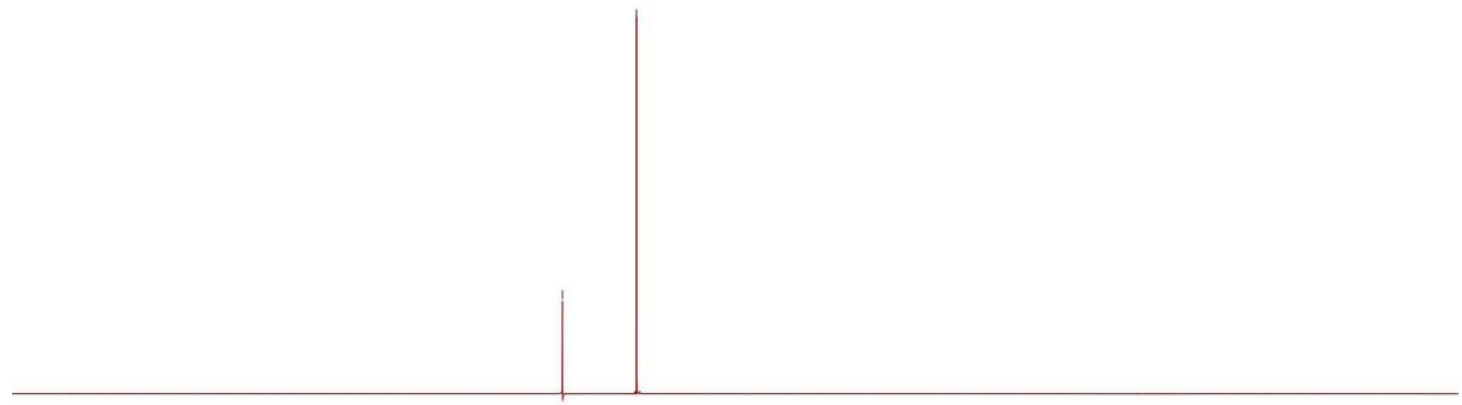

$\begin{array}{llllllllllllllllllllllllllllllll}0 & 10 & 0 & -10 & -20 & -30 & -40 & -50 & -60 & -70 & -80 & -90 & -100 & -110 & -120 & -130 & -140 & -150 & -160 & -170 & -180 & -190 & -200 & -210 & -22\end{array}$

${ }^{1} \mathrm{H}$ NMR (400 MHz, $\mathrm{CDCl}_{3}$ ) spectrum for 3y

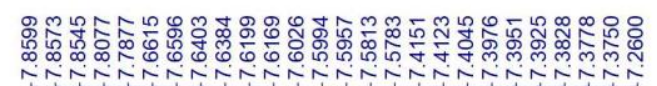<smiles>O=C(c1cc2ccccc2o1)C(F)(F)C(=O)c1cc2ccccc2o1</smiles>

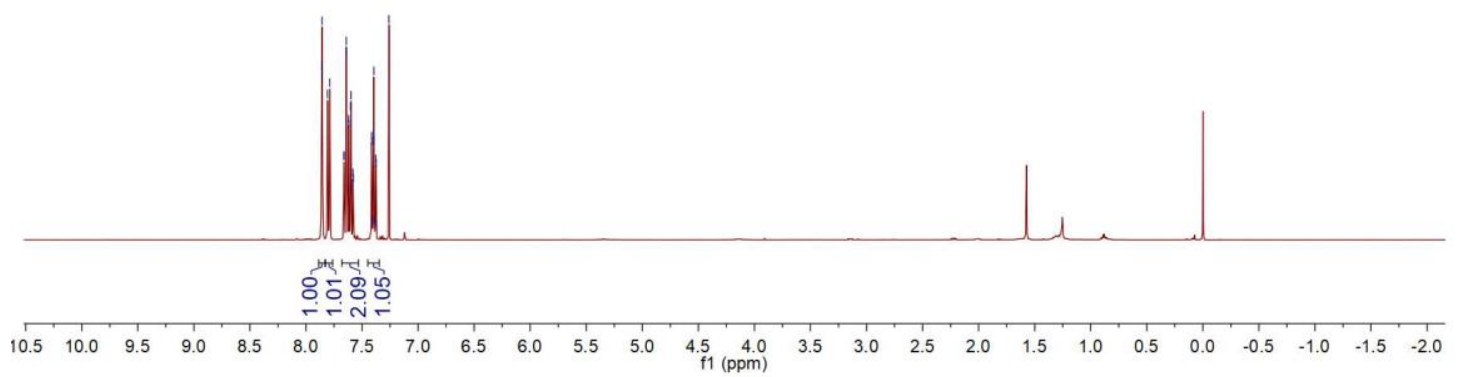


${ }^{13} \mathrm{C}$ NMR (100 MHz, $\left.\mathrm{CDCl}_{3}\right)$ spectrum for $3 y$

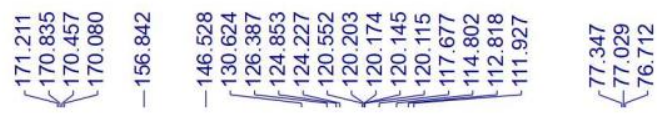
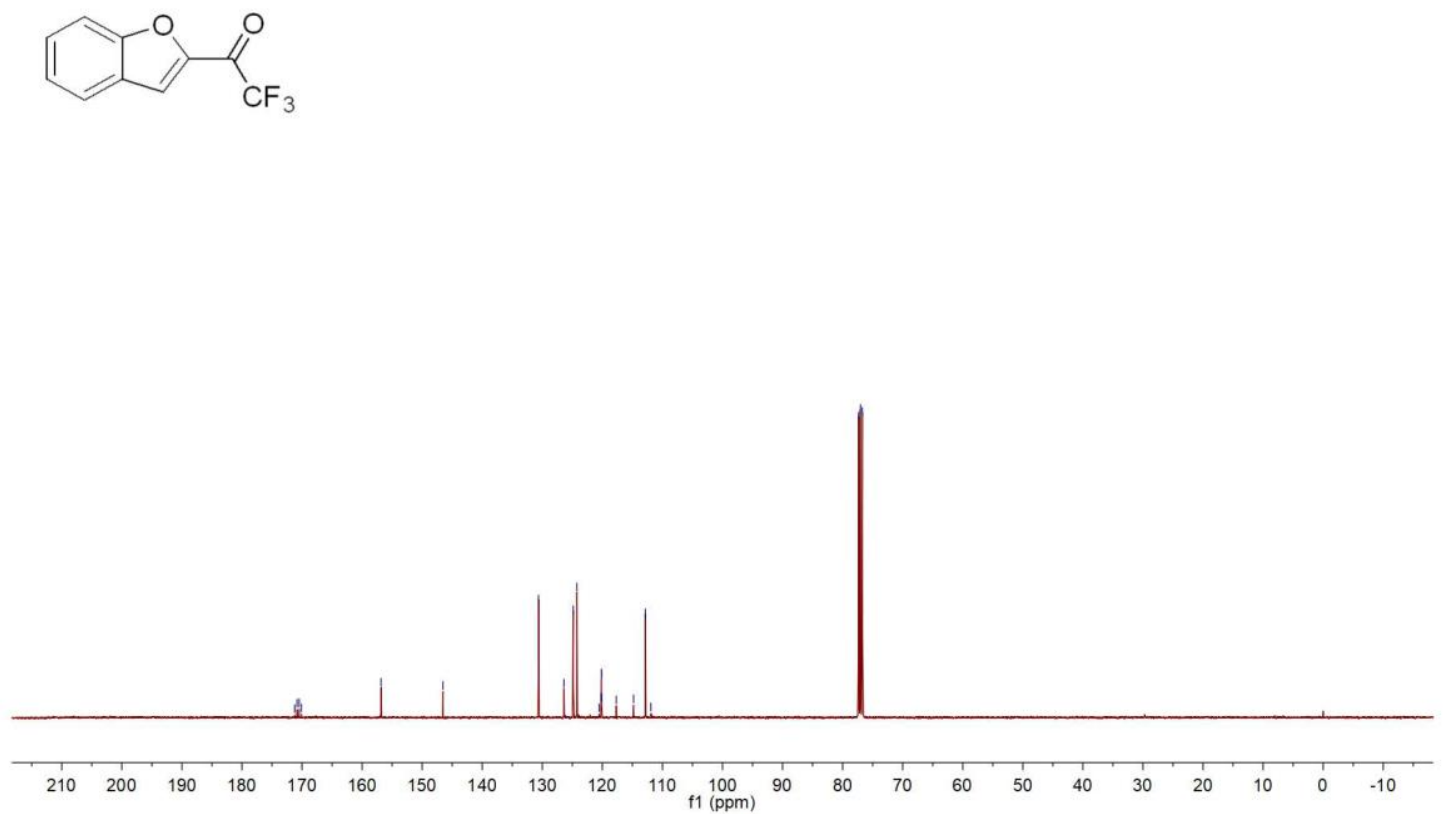

${ }^{19}$ F NMR (376 $\mathrm{MHz}, \mathrm{CDCl}_{3}$ ) spectrum for $3 \mathrm{y}$

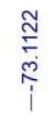<smiles>O=C(c1cc2ccccc2o1)C(F)(F)C(=O)C(F)(F)c1ccccc1</smiles>

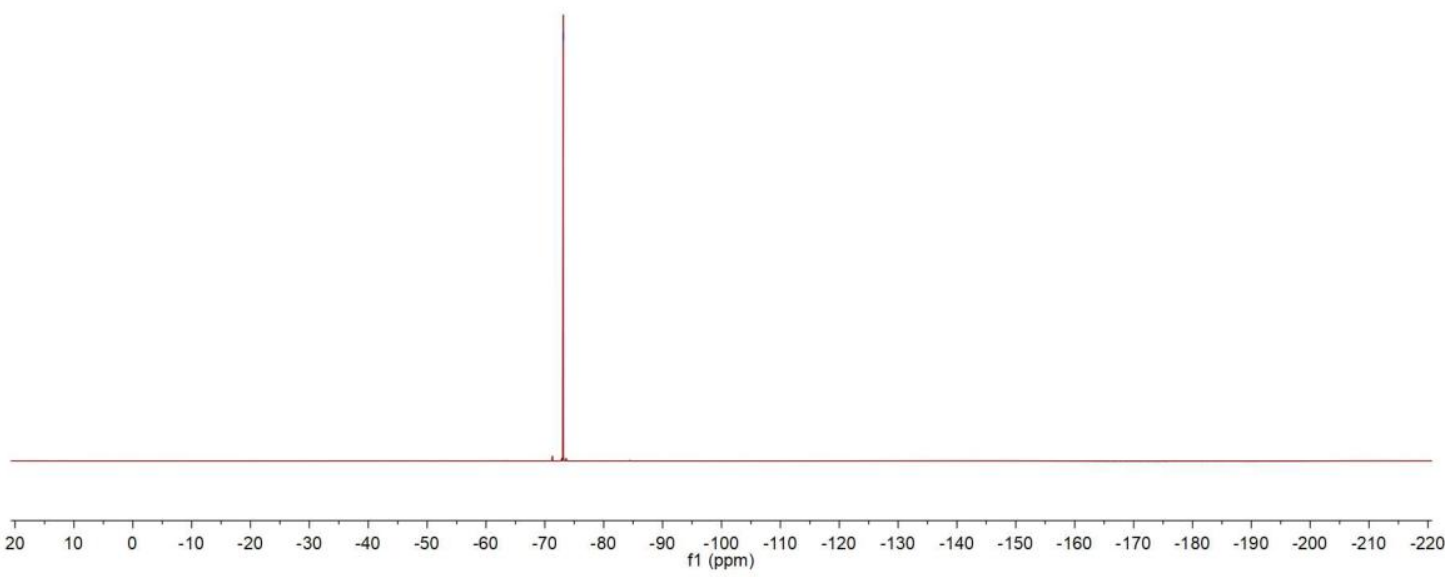


${ }^{1} \mathrm{H}$ NMR (400 MHz, $\mathrm{CDCl}_{3}$ ) spectrum for $3 \mathrm{z}$

m
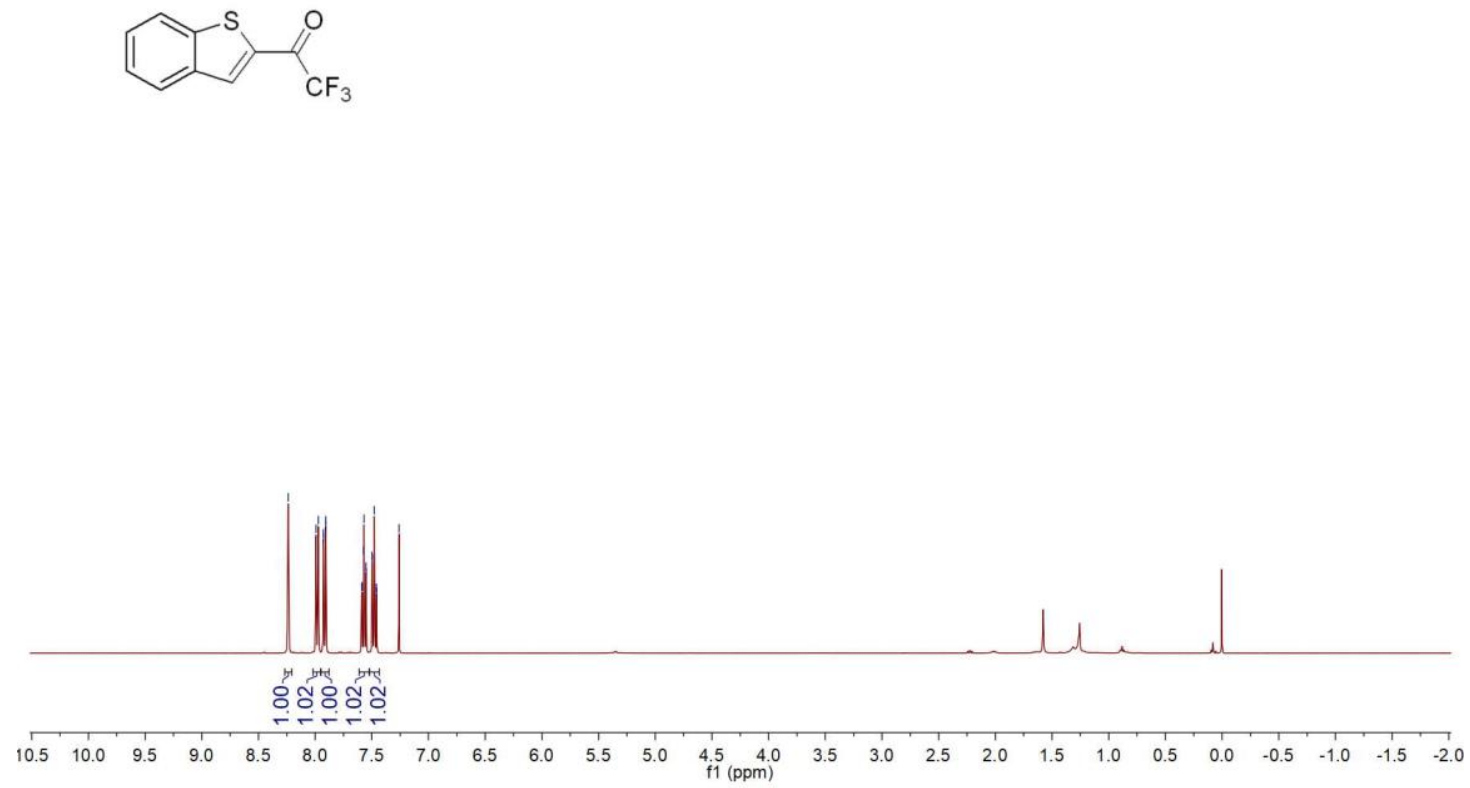

${ }^{13} \mathrm{C}$ NMR (100 MHz, $\mathrm{CDCl}_{3}$ ) spectrum for $3 \mathrm{z}$

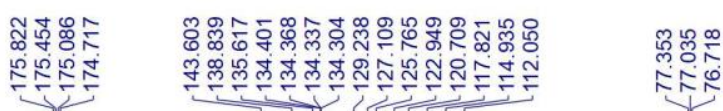
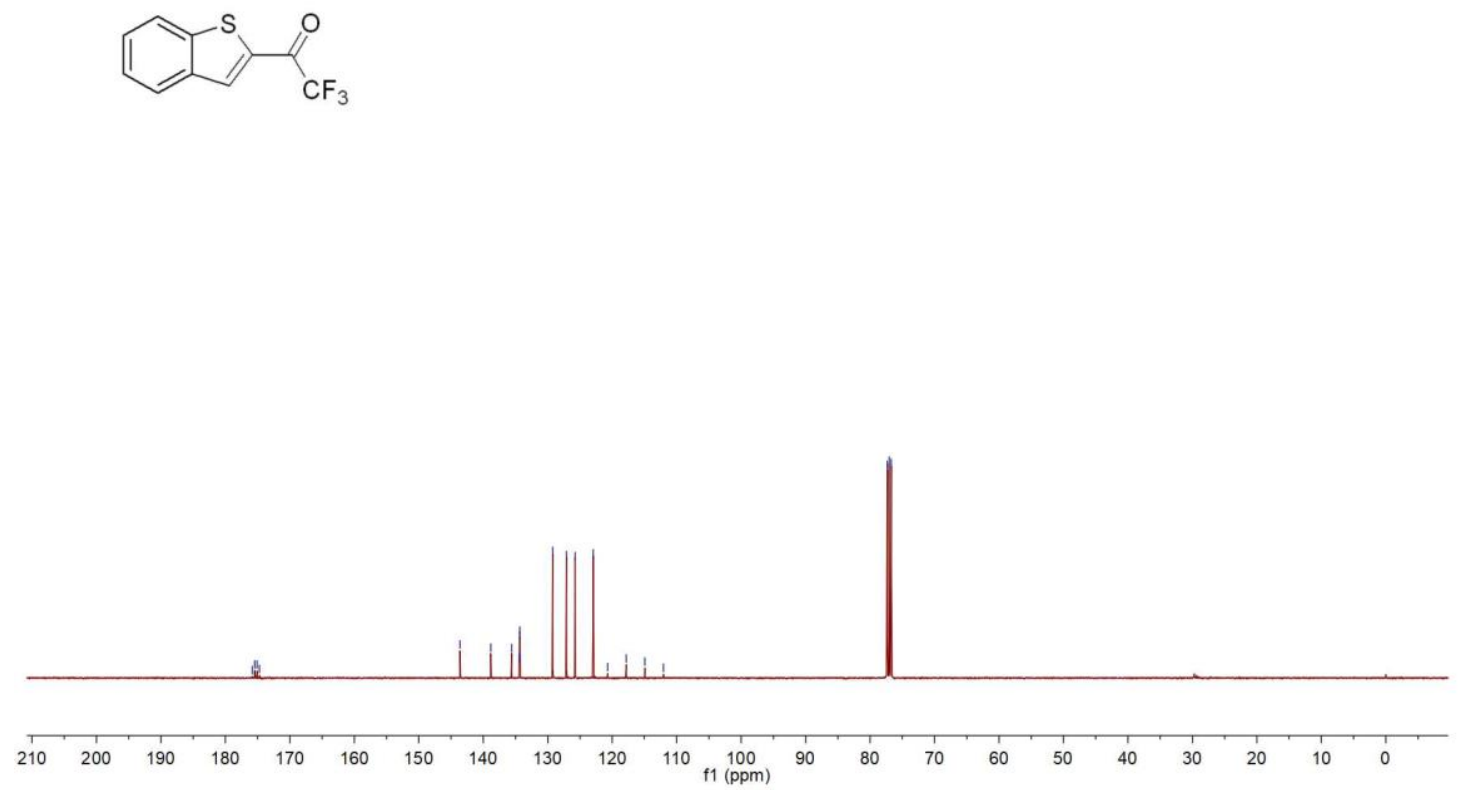
${ }^{19}$ F NMR (376 MHz, $\mathrm{CDCl}_{3}$ ) spectrum for $3 \mathrm{z}$

$$
\text { ণั }
$$<smiles>O=C(c1cc2ccccc2s1)C(F)(F)F</smiles>

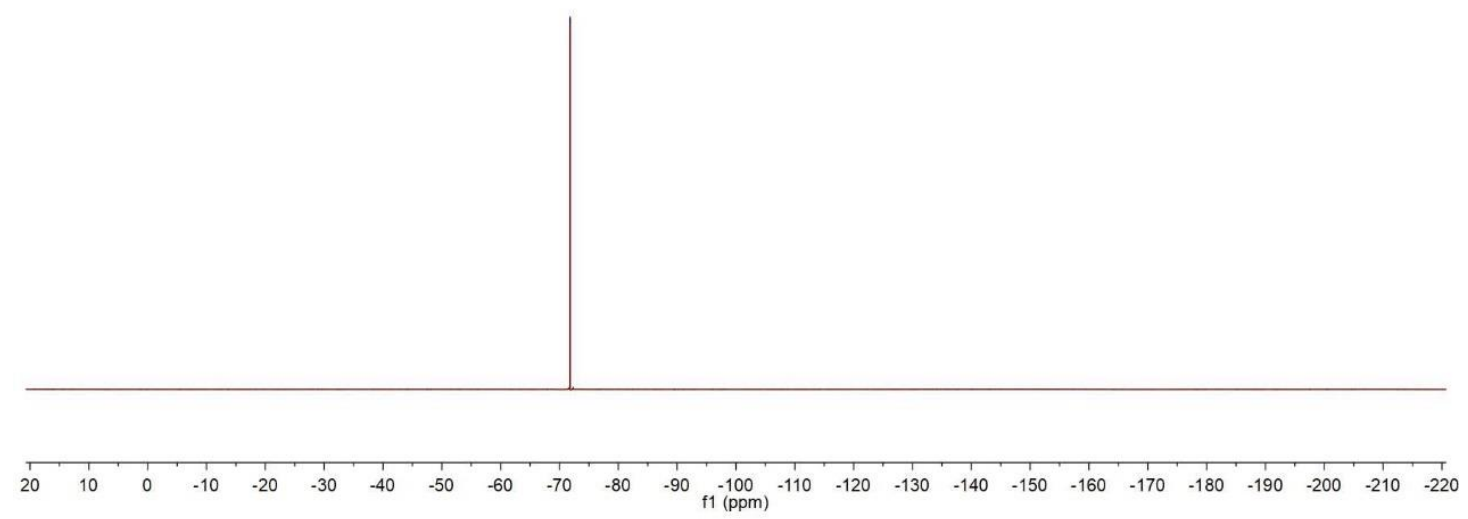

${ }^{1} \mathrm{H}$ NMR (400 MHz, $\mathrm{CDCl}_{3}$ ) spectrum for 3aa

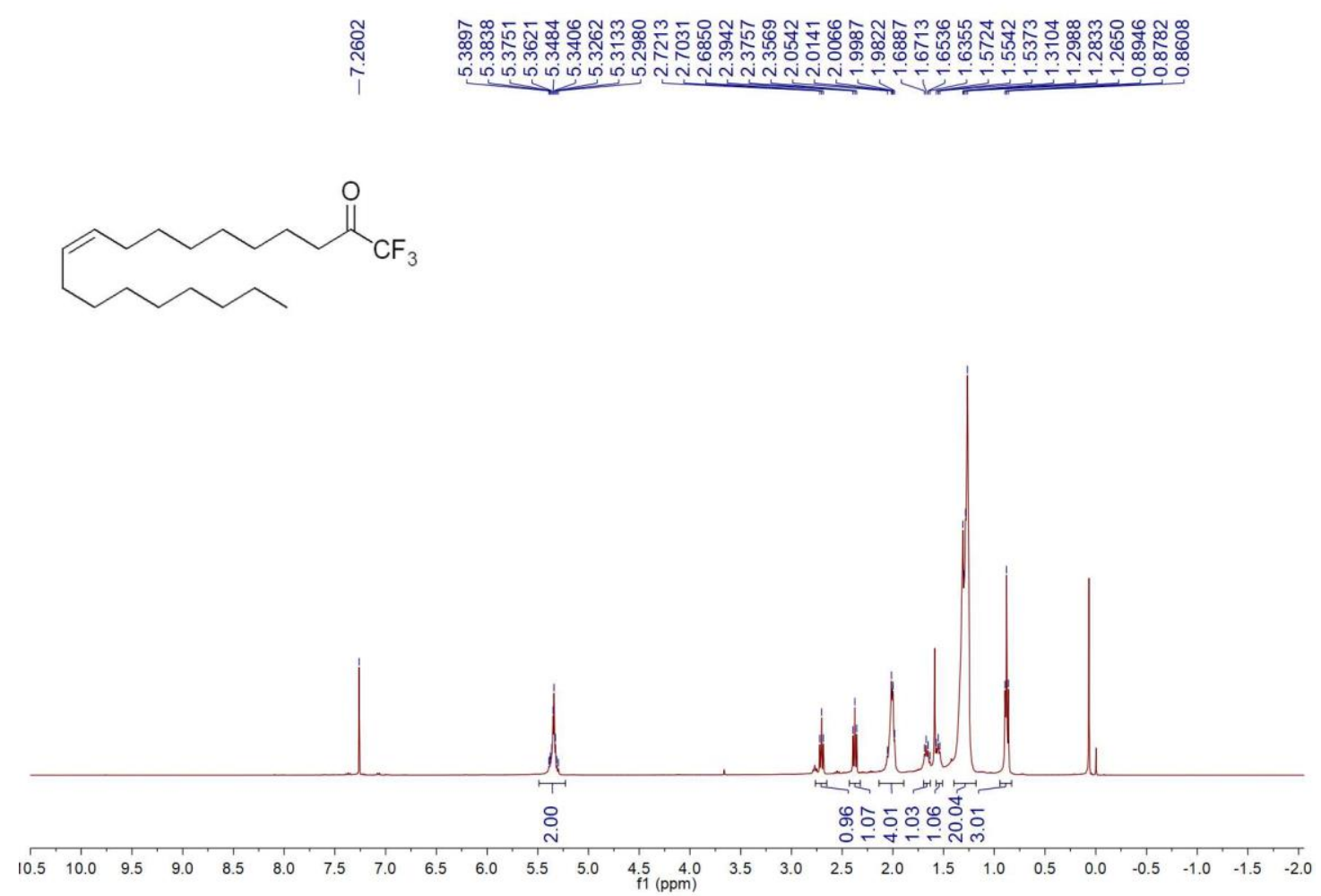


${ }^{13} \mathrm{C}$ NMR (100 MHz, $\mathrm{CDCl}_{3}$ ) spectrum for $3 \mathrm{aa}$

下孚泪

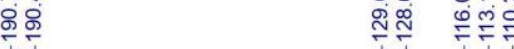

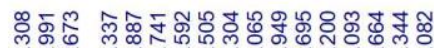

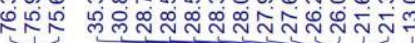<smiles>CCCCCCCCCCCCC(=O)C(C)(C)C</smiles>

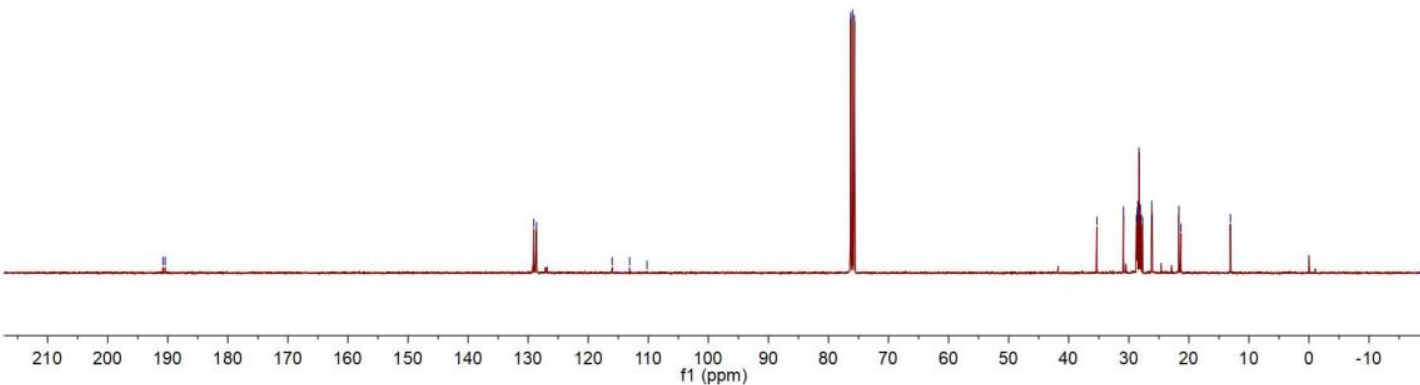

${ }^{19}$ F NMR (376 $\mathrm{MHz}, \mathrm{CDCl}_{3}$ ) spectrum for 3aa

畩<smiles>CCCCCCCCCCCCC(=O)C(C)(C)C</smiles>

$\begin{array}{lllllllllllllllllllllllllllll}20 & 10 & 0 & -10 & -20 & -30 & -40 & -50 & -60 & -70 & -80 & -90 & -10 & -110 & -120 & -130 & -140 & -150 & -160 & -170 & -180 & -190 & -200 & -210 & -220\end{array}$ 
${ }^{1} \mathrm{H}$ NMR (400 MHz, $\mathrm{CDCl}_{3}$ ) spectrum for 3ab<smiles>CCCCCCCCCCCCCCCC(=O)C(F)(F)F</smiles>

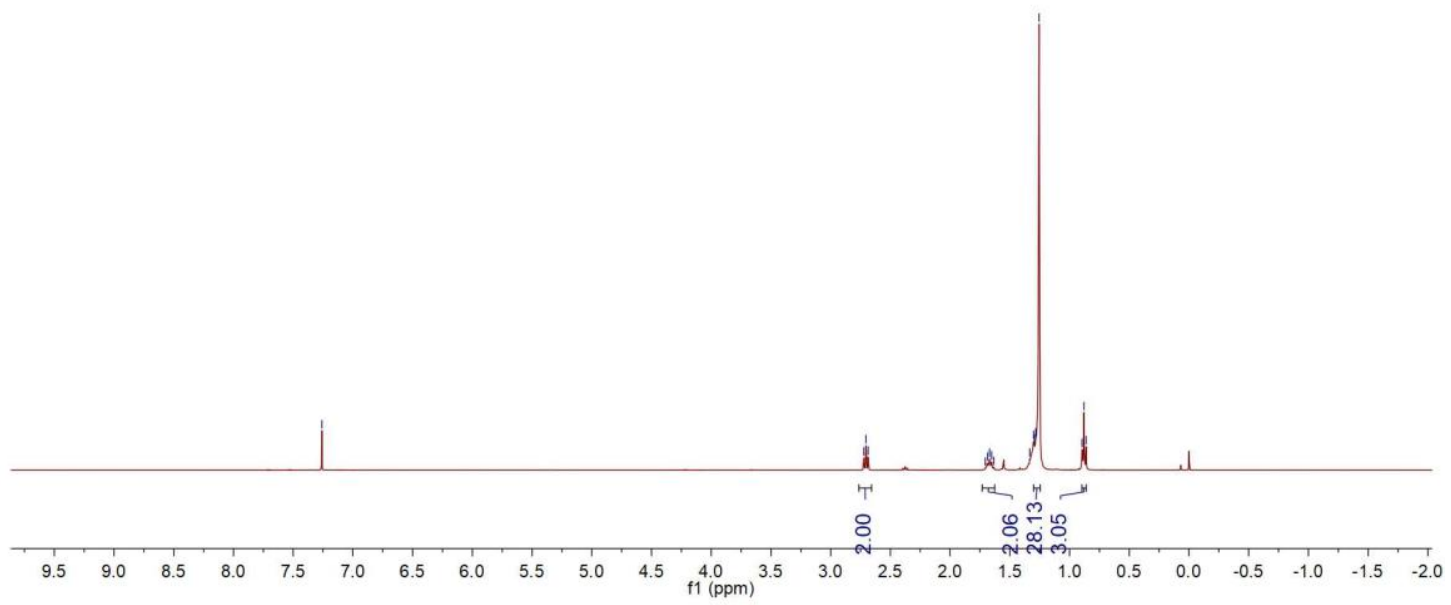

${ }^{13} \mathrm{C}$ NMR (100 $\mathrm{MHz}, \mathrm{CDCl}_{3}$ ) spectrum for 3ab

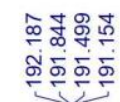

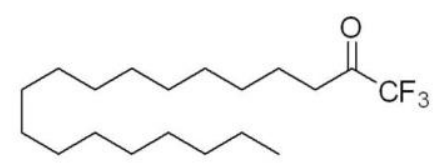

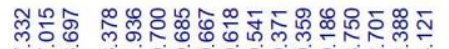

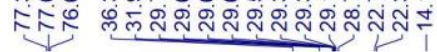


${ }^{19}$ F NMR (376 MHz, CDCl 3 ) spectrum for 3ab

志<smiles>CCCCCCCCCCCCCCCCC(=O)C(F)(F)F</smiles>

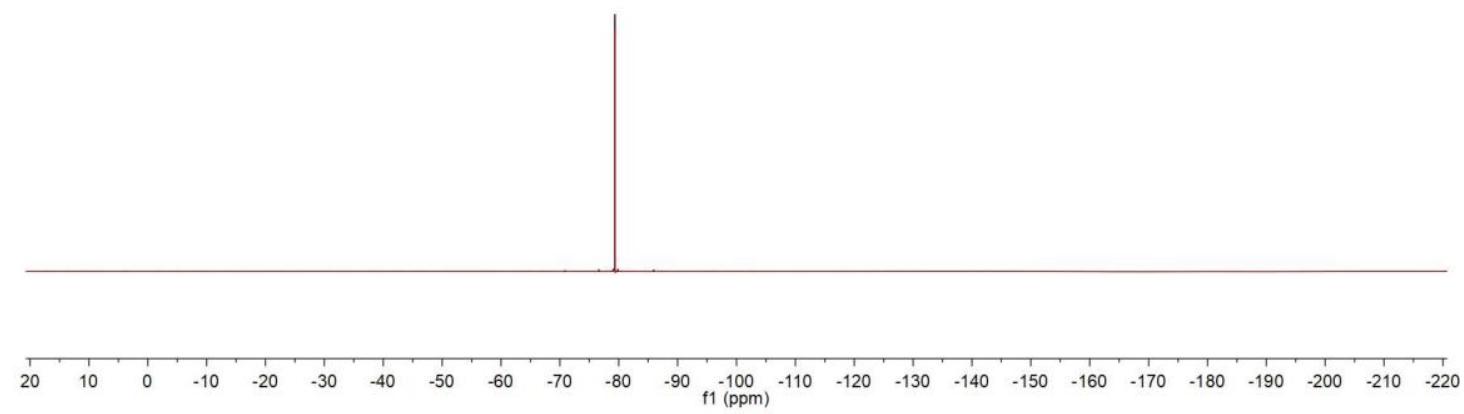

${ }^{1} \mathrm{H}$ NMR (400 MHz, $\mathrm{CDCl}_{3}$ ) spectrum for 3ac

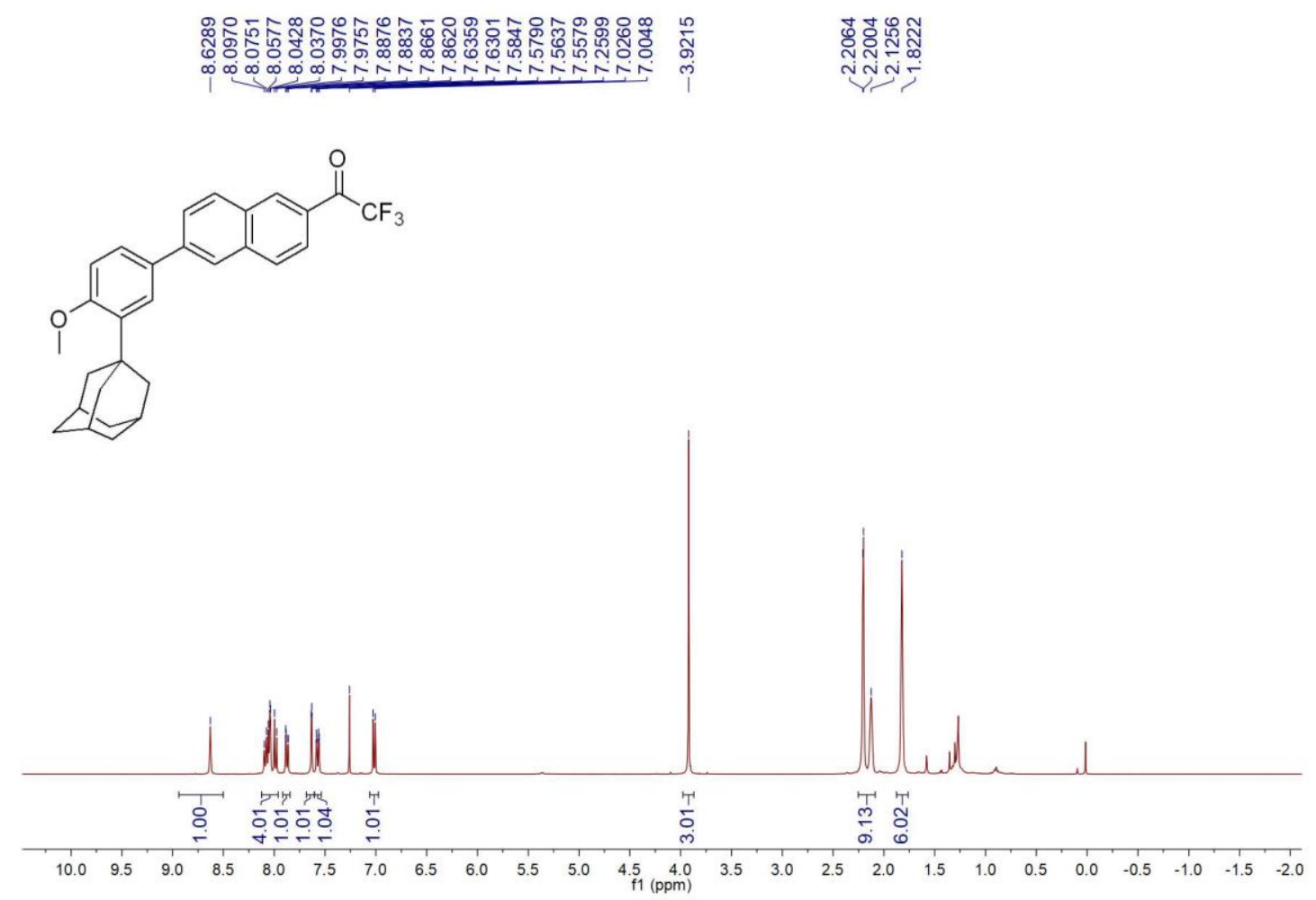


${ }^{13} \mathrm{C}$ NMR (100 MHz, $\mathrm{CDCl}_{3}$ ) spectrum for 3ac

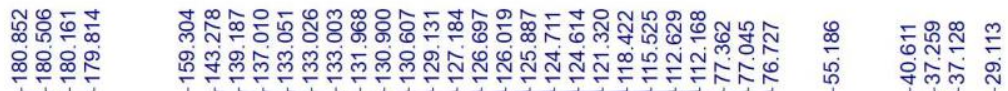<smiles>COc1ccc(-c2ccc3cc(C(=O)C(F)(F)F)ccc3c2)cc1C12CCCC(CC1)C2</smiles>

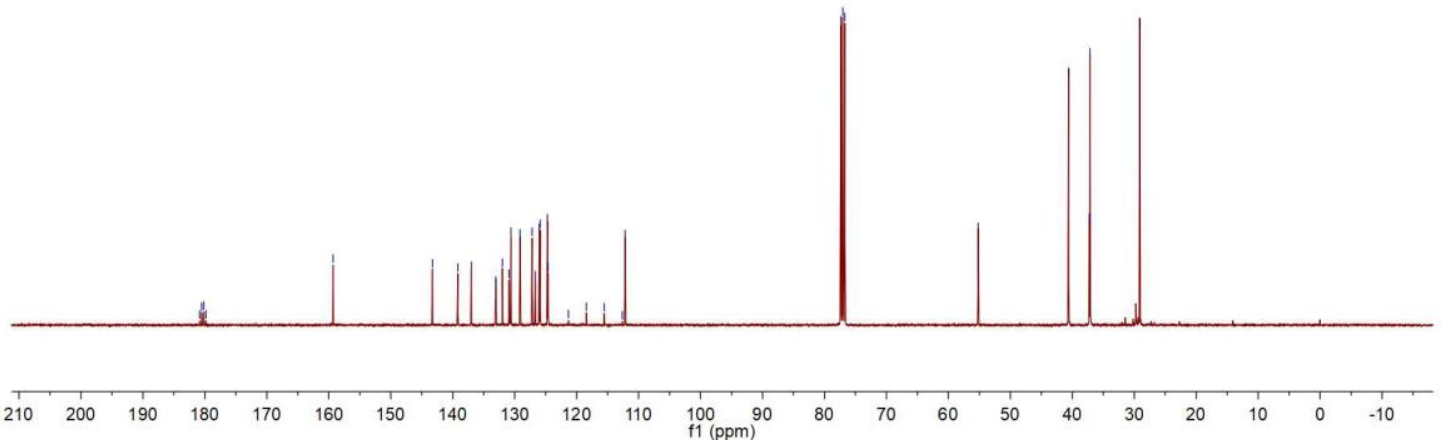

${ }^{19} \mathrm{~F}$ NMR (376 $\mathrm{MHz}, \mathrm{CDCl}_{3}$ ) spectrum for $3 \mathrm{ac}$<smiles>C1CCCCC1</smiles><smiles>COc1ccc(-c2ccc3cc(C(=O)C(F)(F)F)ccc3c2)cc1C12CC3CC(CC(C3)C1)C2</smiles>

$\begin{array}{llllllllllllllllllllllllllllllllll}20 & 10 & 0 & -10 & -20 & -30 & -40 & -50 & -60 & -70 & -80 & -90 & -100 & -110 & 120 & -130 & -140 & -150 & -160 & -170 & -180 & -190 & -200 & -210 & -220\end{array}$ 
${ }^{1} \mathrm{H}$ NMR (400 MHz, $\mathrm{CDCl}_{3}$ ) spectrum for 3ad

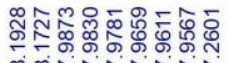
oninisinis<smiles>CCCN(CCC)S(=O)(=O)c1ccc(C(=O)C(F)(F)F)cc1</smiles>

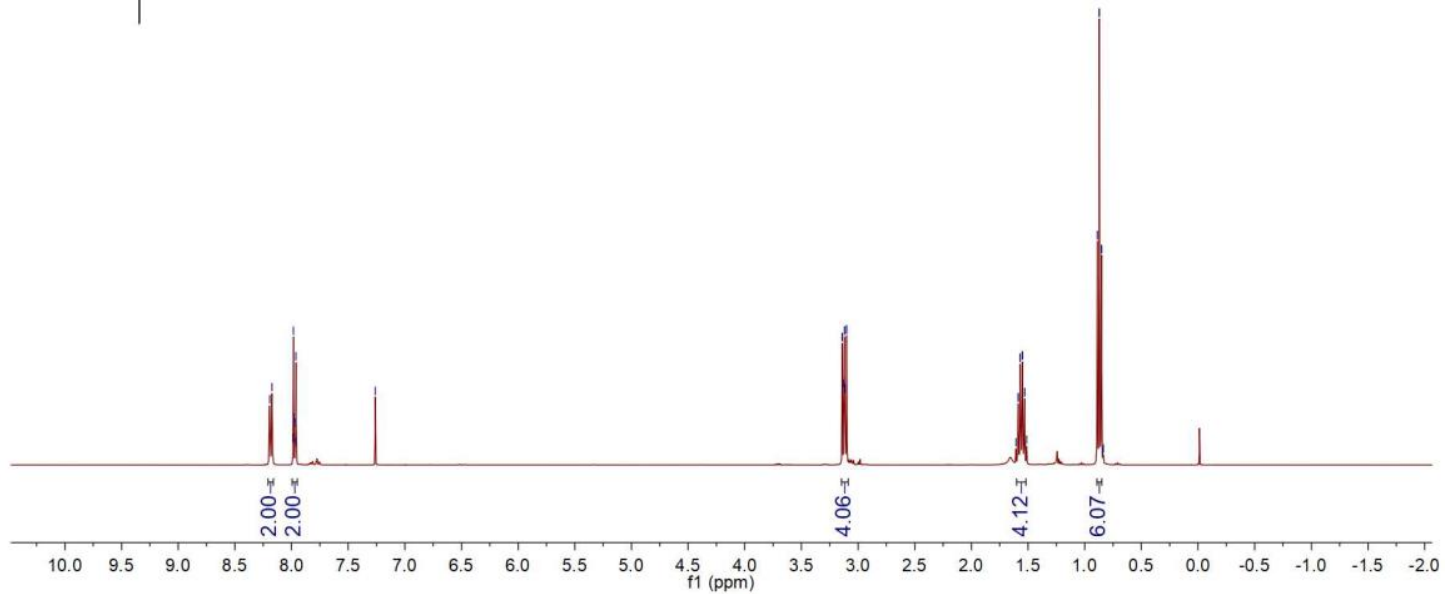

${ }^{13} \mathrm{C}$ NMR (100 MHz, $\mathrm{CDCl}_{3}$ ) spectrum for 3ad
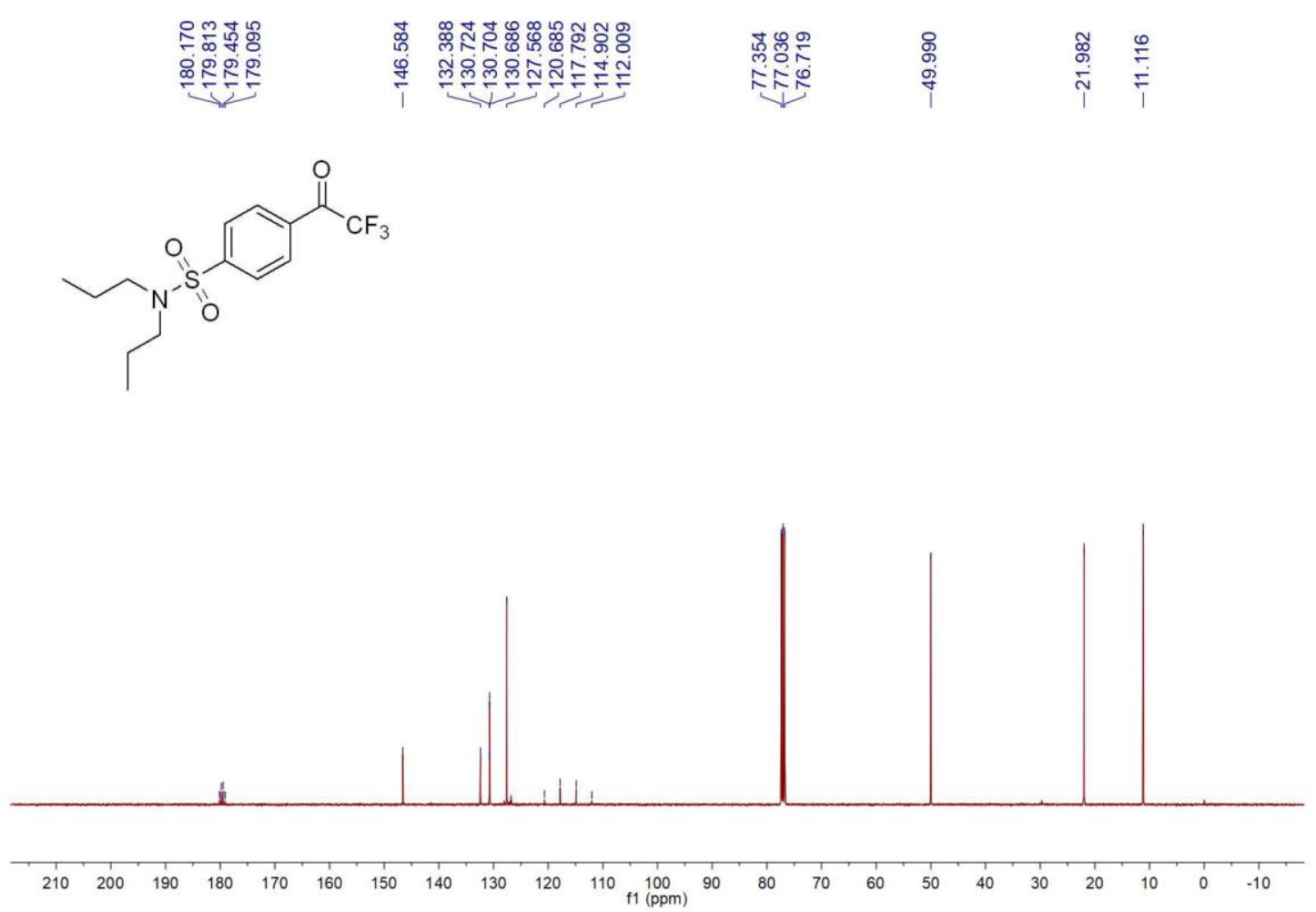
${ }^{19}$ F NMR (376 MHz, CDCl $)$ spectrum for 3ad

产<smiles>CCCN(CCC)S(=O)(=O)c1ccc(C(=O)C(F)(F)F)cc1</smiles>

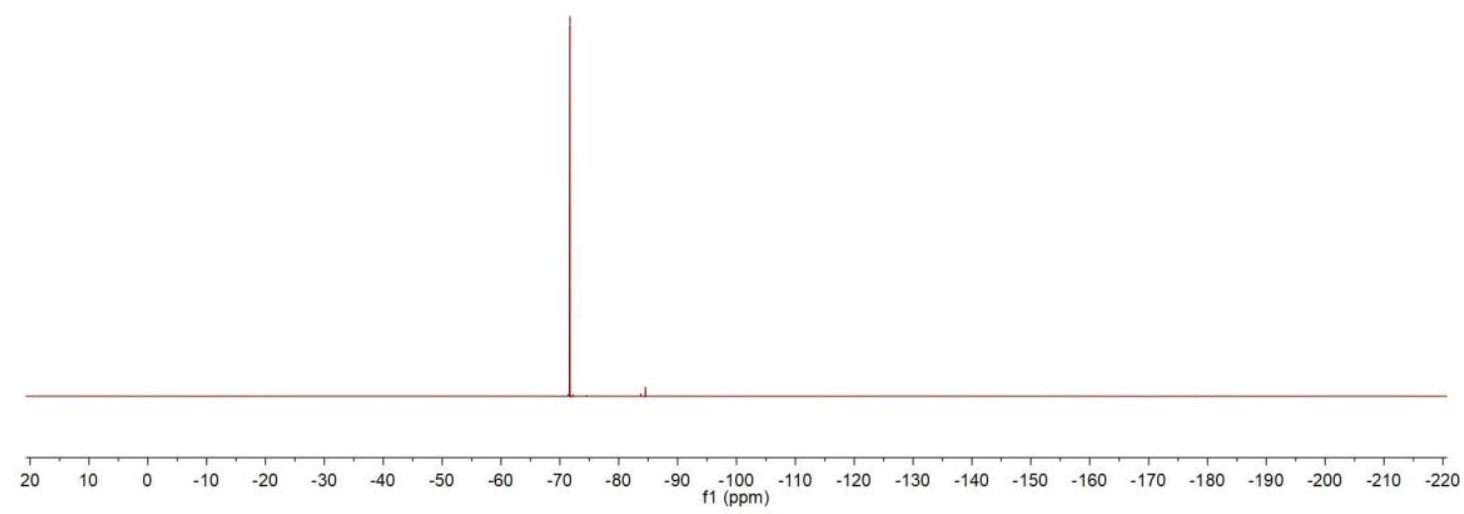

${ }^{1} \mathrm{H}$ NMR (400 MHz, $\mathrm{CDCl}_{3}$ ) spectrum for 3ae

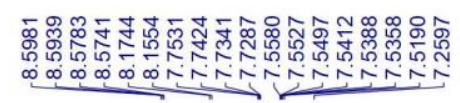<smiles>Cc1c(-c2ccccc2)oc2c(C(=O)C(F)(F)F)cccc2c1=O</smiles>

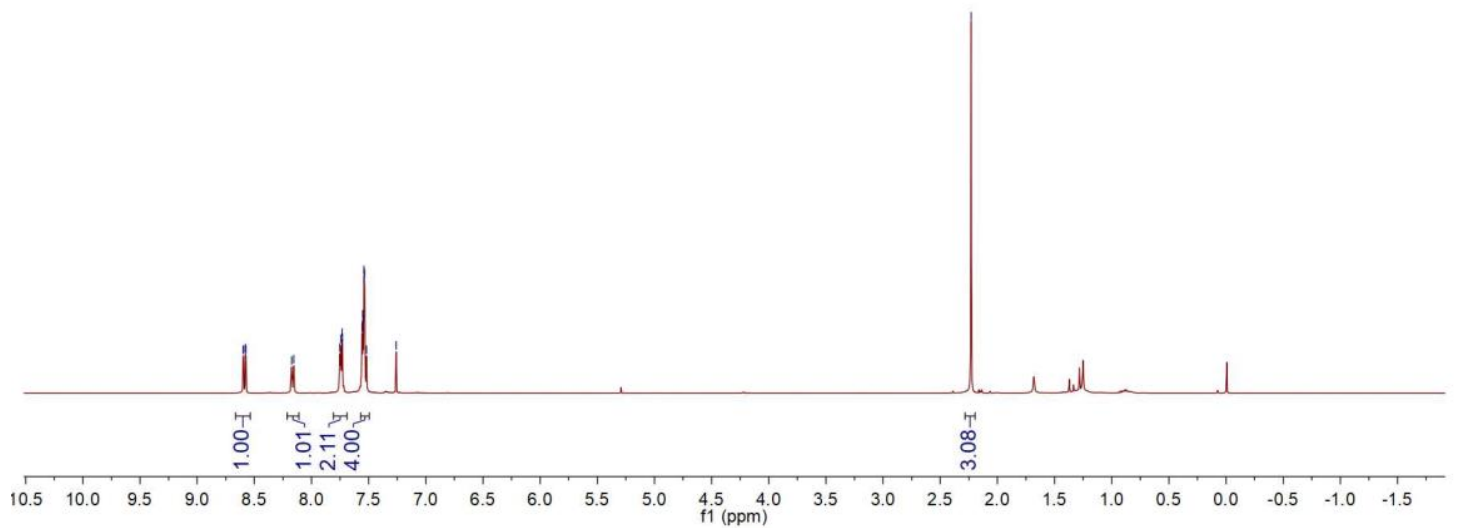


${ }^{13} \mathrm{C}$ NMR (100 MHz, $\mathrm{CDCl}_{3}$ ) spectrum for 3ae

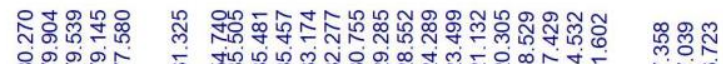

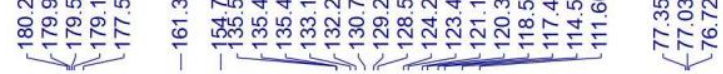

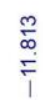<smiles>Cc1c(-c2ccccc2)oc2c(C(=O)C(F)(F)F)cccc2c1=O</smiles>

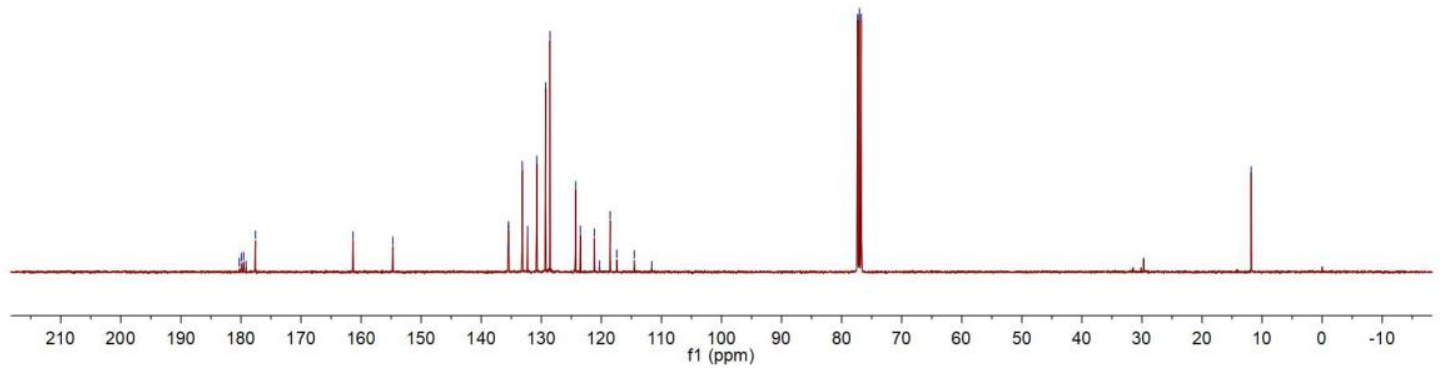

${ }^{19} \mathrm{~F}$ NMR (376 $\mathrm{MHz}, \mathrm{CDCl}_{3}$ ) spectrum for 3ae

온<smiles>Cc1c(-c2ccccc2)oc2c(C(=O)C(F)(F)F)cccc2c1=O</smiles>

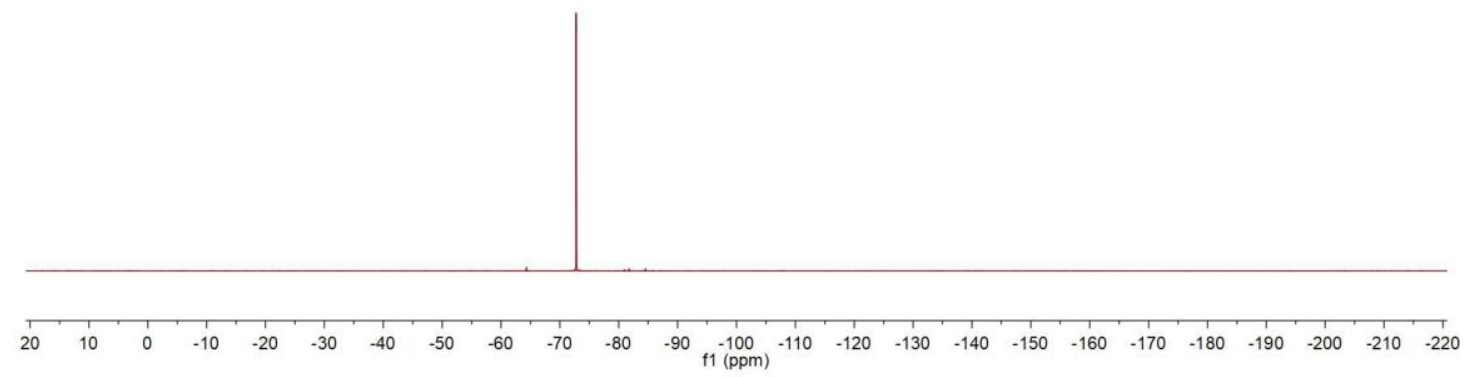


${ }^{1} \mathrm{H}$ NMR (400 MHz, $\left.\mathrm{CDCl}_{3}\right)$ spectrum for 3af

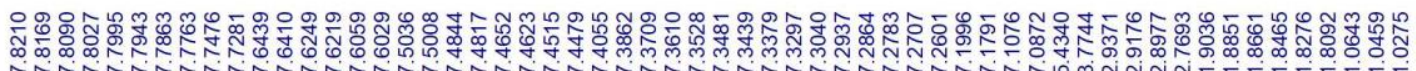
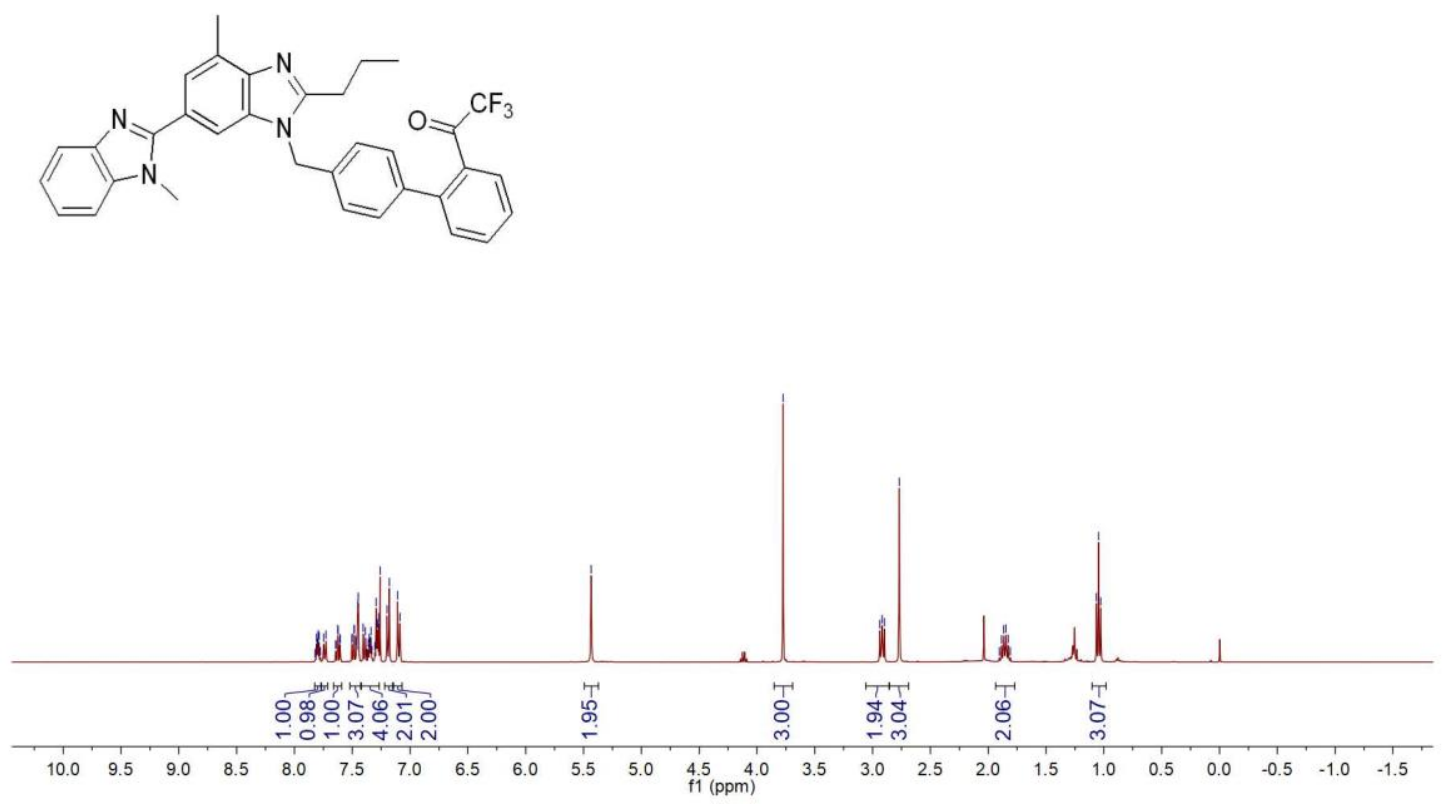

${ }^{13} \mathrm{C}$ NMR (100 MHz, $\left.\mathrm{CDCl}_{3}\right)$ spectrum for 3af

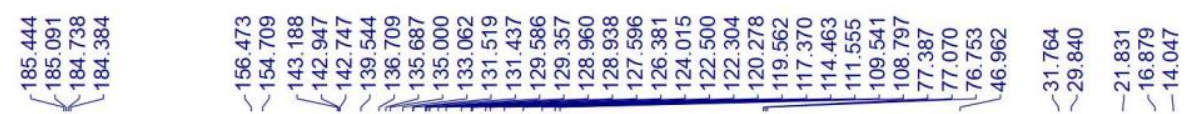<smiles>CCCc1nc2c(C)cc(-c3nc4ccccc4n3C)cc2n1Cc1ccc(-c2ccccc2C(=O)C(F)(F)F)cc1</smiles>

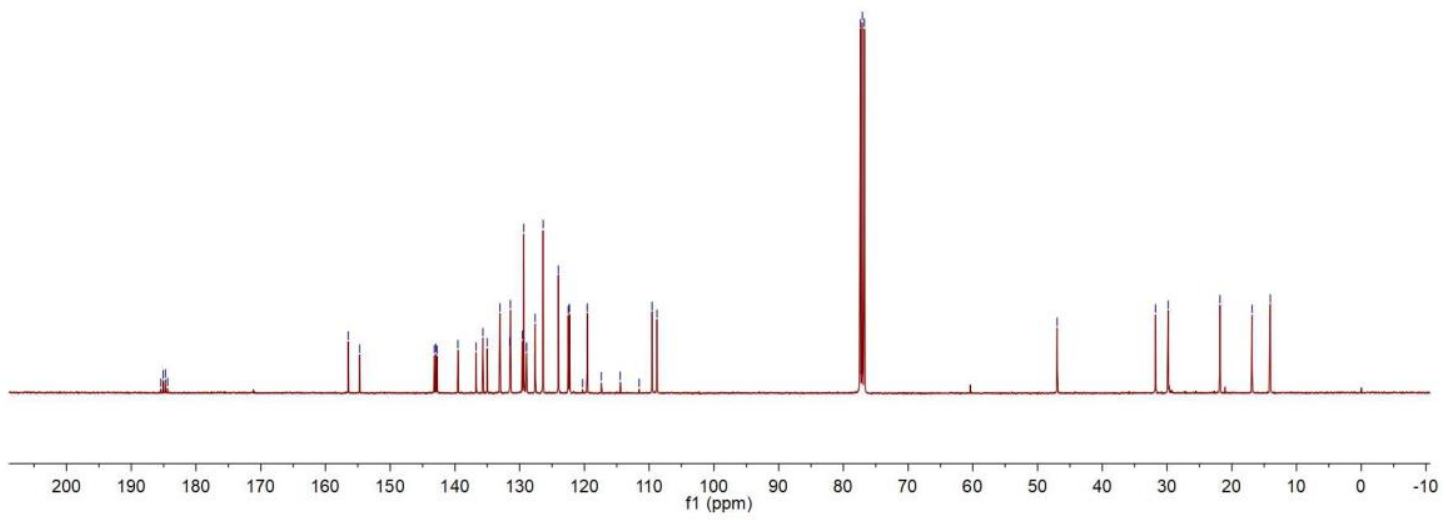


${ }^{19}$ F NMR (376 MHz, $\mathrm{CDCl}_{3}$ ) spectrum for 3af<smiles>CCCc1nc2c(C)cc(-c3nc4ccccc4n3C)cc2n1Cc1ccc(-c2ccccc2C(=O)C(F)(F)F)cc1</smiles>

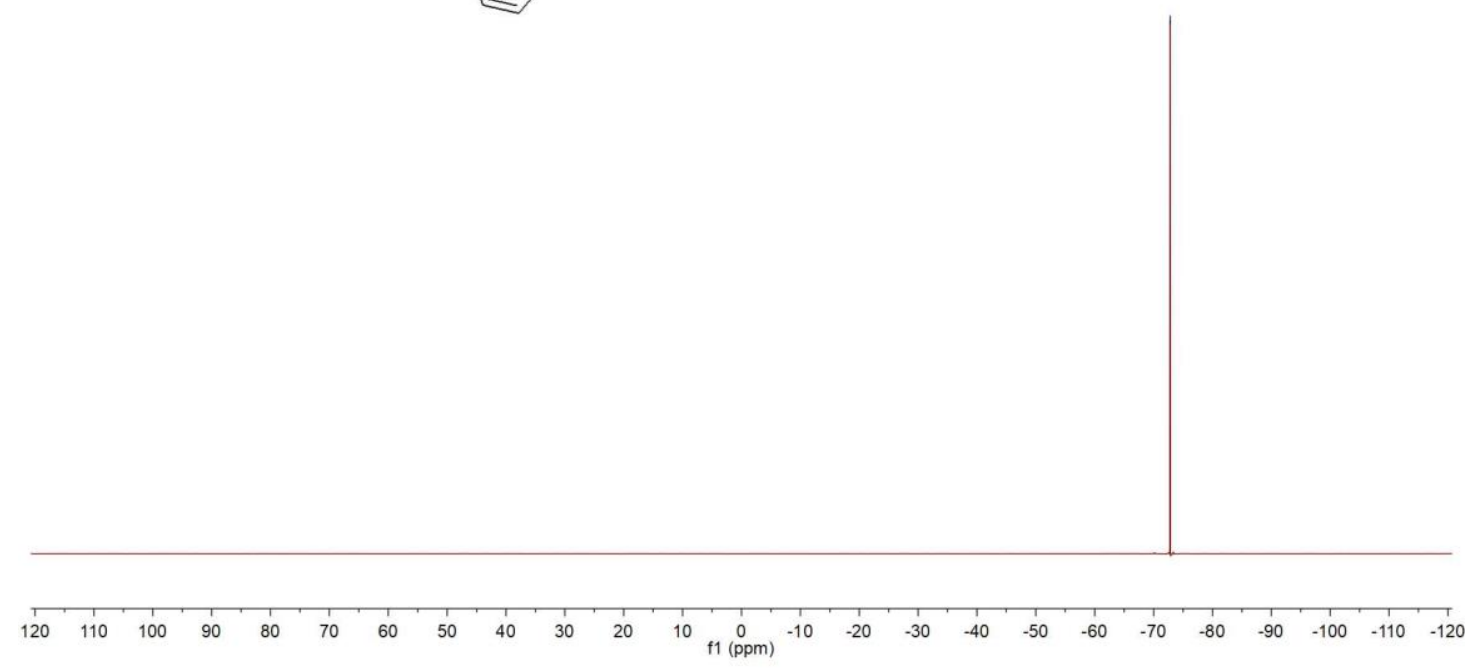

